Aus dem Geriatrischen Zentrum

(Prof. Dr. med. R. Nau)

des Evangelischen Krankenhauses Göttingen-Weende

\title{
Psychopharmaka und das Risiko von Stürzen in der stationären geriatrischen Versorgung
}

\author{
INAUGURAL-DISSERTATION \\ zur Erlangung des Doktorgrades \\ der Medizinischen Fakultät der \\ Georg-August-Universität zu Göttingen
}

\author{
vorgelegt von \\ Fabian Max Wedmann \\ aus
}

Hildesheim

Göttingen 2018 
Dekan:

\section{Betreuungsausschuss}

Betreuer/in

Ko-Betreuer/in:

\section{Prüfungskommission}

Referent/in

Ko-Referent/in:

Drittreferent/in:

Datum der mündlichen Prüfung:
Prof. Dr. rer. nat. H. K. Kroemer

Prof. Dr. med. R. Nau

Prof. W. Himmel 
Hiermit erkläre ich, die Dissertation mit dem Titel "Psychopharmaka und das Risiko von Stürzen in der stationären geriatrischen Versorgung" eigenständig angefertigt und keine anderen als die von mir angegebenen Quellen und Hilfsmittel verwendet zu haben.

Göttingen, den

(Unterschrift) 


\section{Inhaltsverzeichnis}

Abbildungsverzeichnis ....................................................................................... III

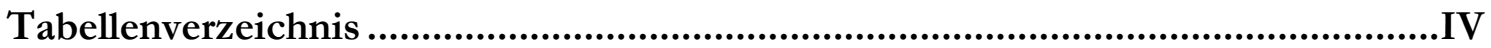

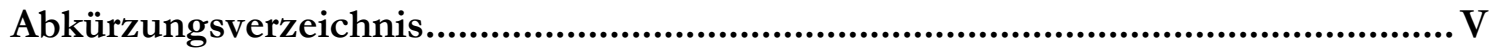

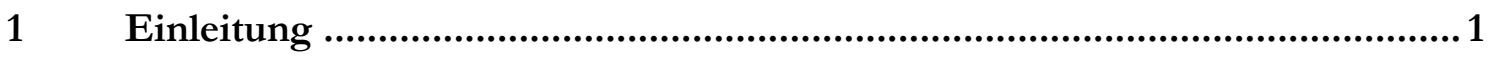

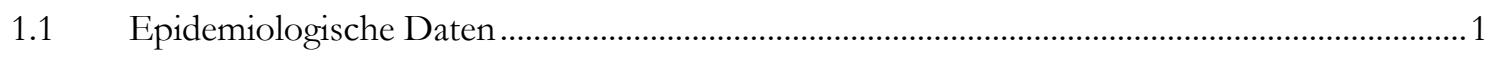

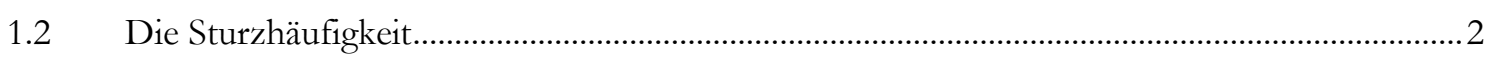

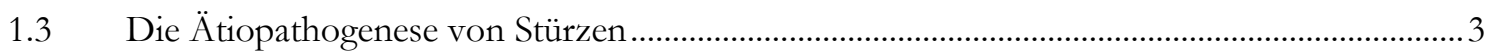

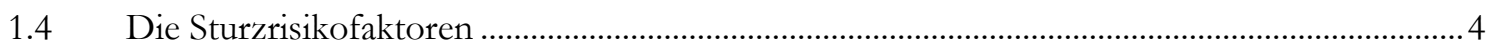

1.4.1 Kognitive Fähigkeiten..........................................................................................................

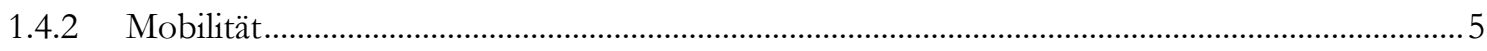

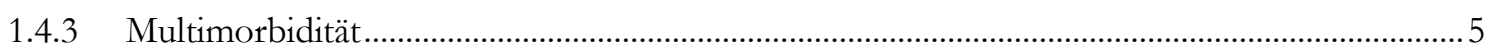

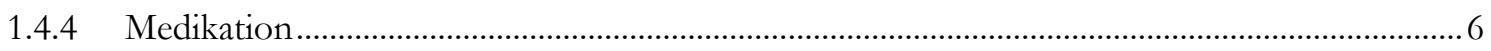

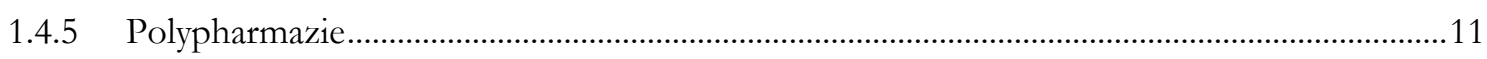

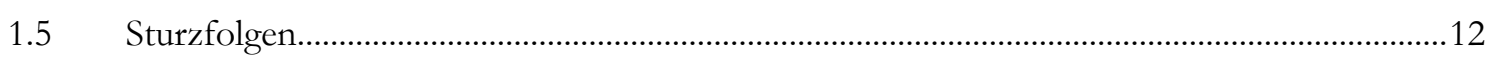

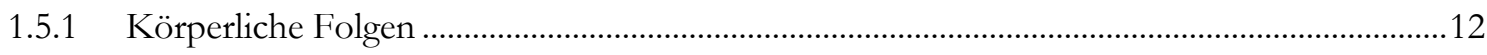

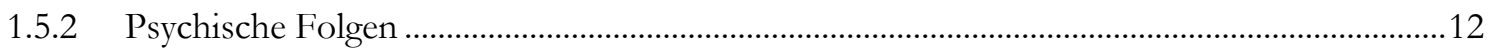

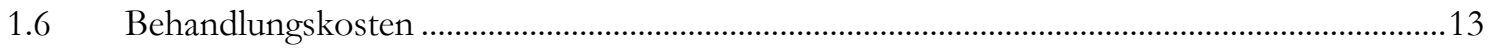

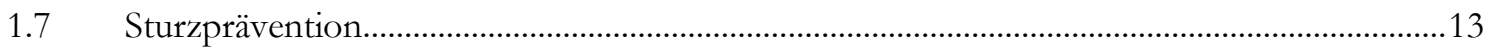

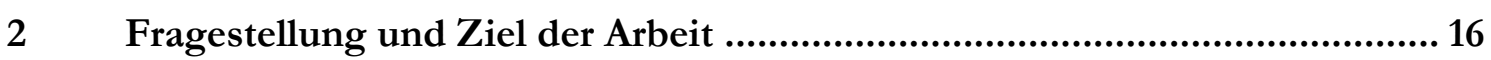

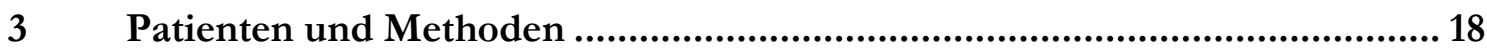

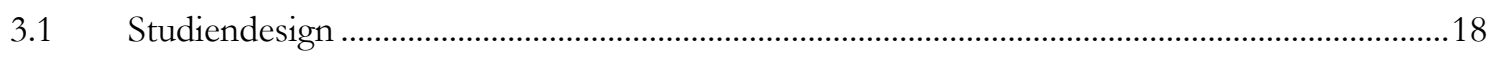

3.2 Stellenwert der Untersuchung im Gesamtprojekt......................................................................18

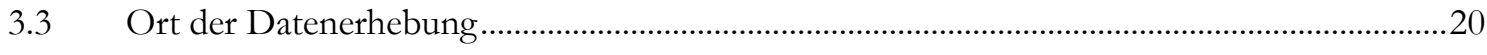

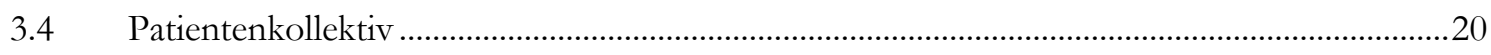

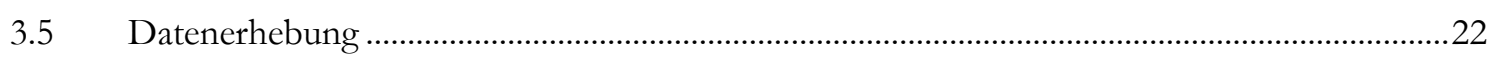

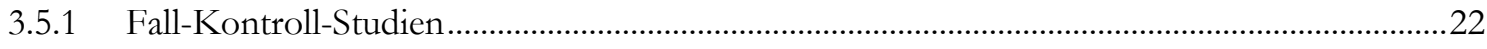

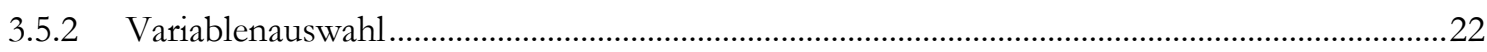

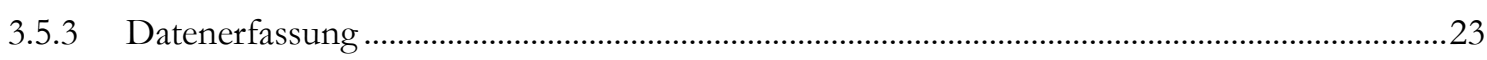

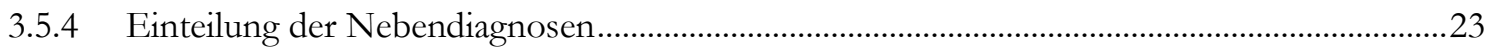

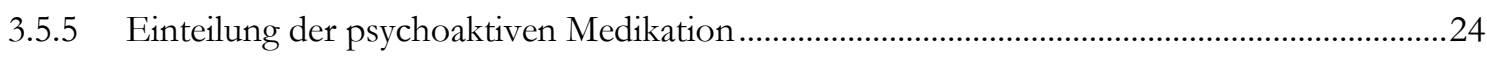

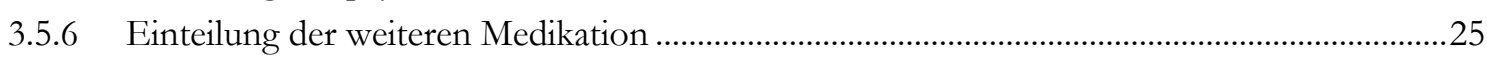

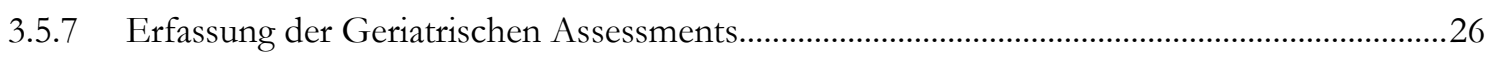

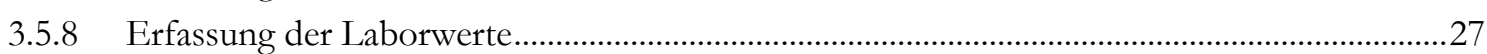

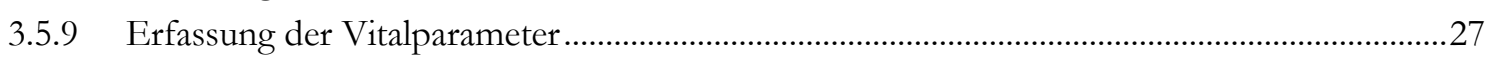

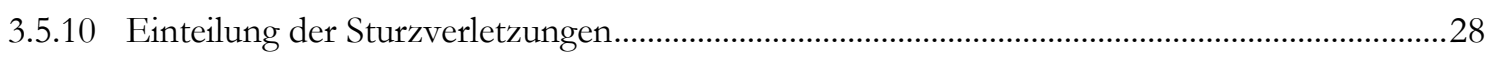

3.5.11 Erfassung der Medikation der Kontrollgruppe ......................................................................28 


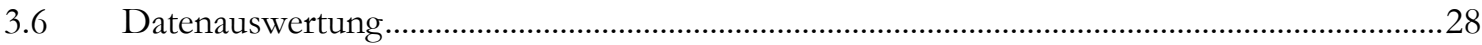

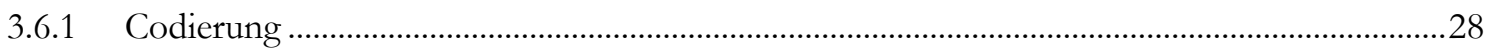

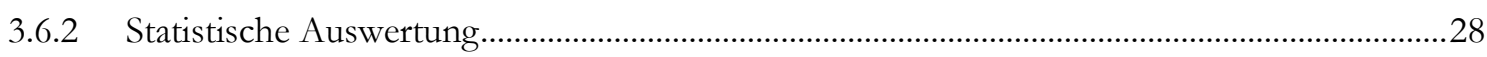

3.7 Datenschutz und Genehmigung durch die Ethikkomission .................................................... 31

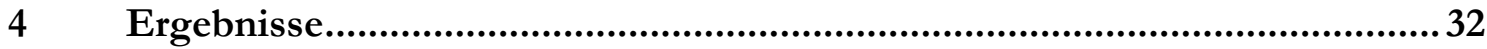

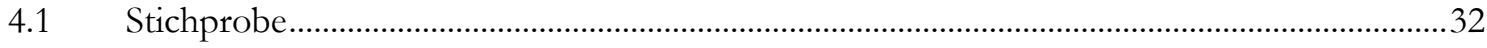

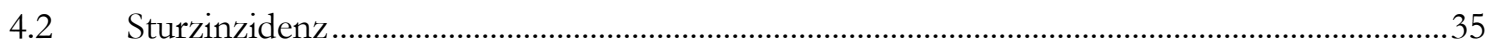

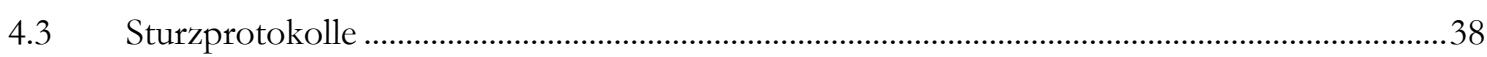

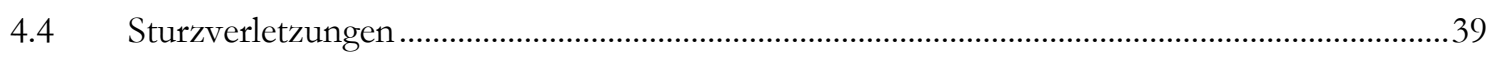

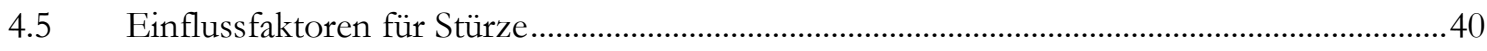

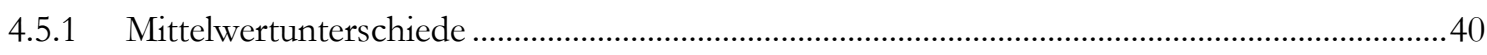

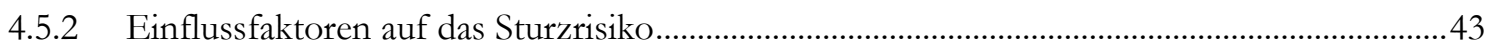

4.5.3 Einfluss von Menge und Dosis ausgewählter Medikamente auf das Sturzrisiko....................50

$5 \quad$ Diskussion........................................................................................55

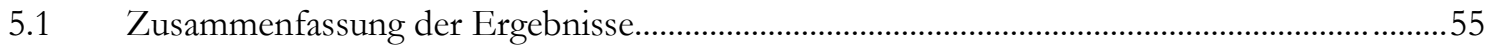

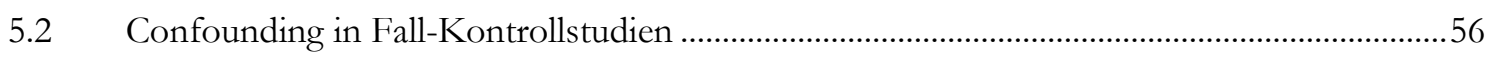

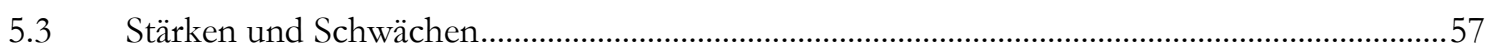

5.4 Medikamente und medizinische Diagnosen als Risikofaktoren für Stürze, eine kritische Auseinandersetzung im Kontext unerwünschter Arzneimittelnebenwirkungen- und

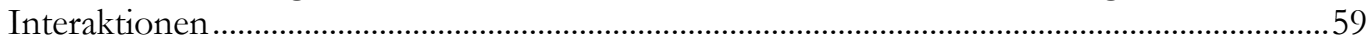

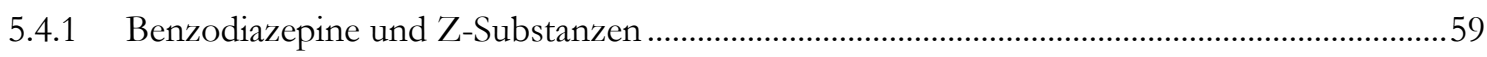

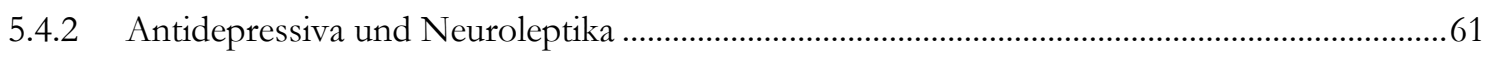

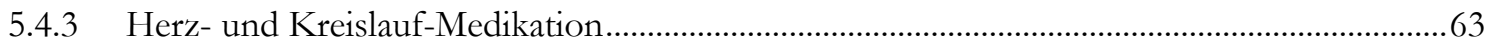

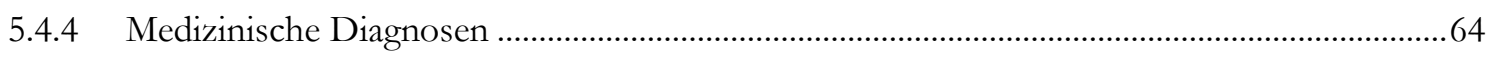

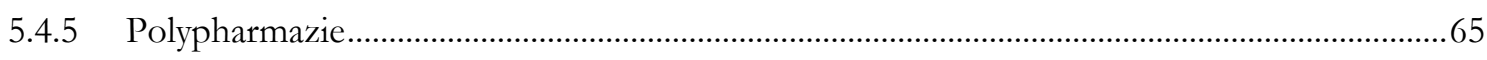

5.5 Wie können Sturzpatienten identifiziert werden und sind geriatrische Assessments zur

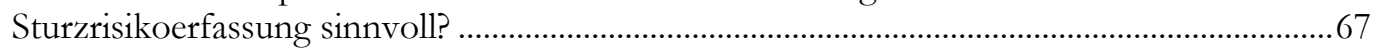

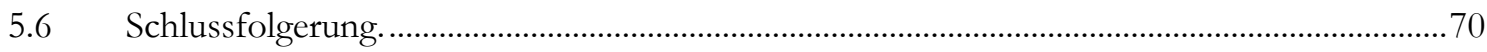

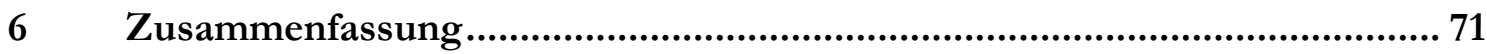

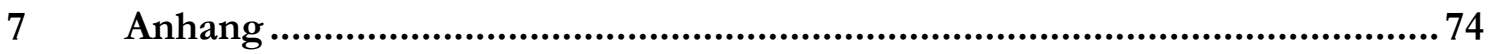

$8 \quad$ Literaturverzeichnis ................................................................. 91 


\section{Abbildungsverzeichnis}

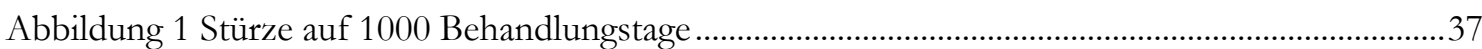

Abbildung 2 Sturzursachen nach behandelnder Abteilung 2014 ............................................................39 


\section{Tabellenverzeichnis}

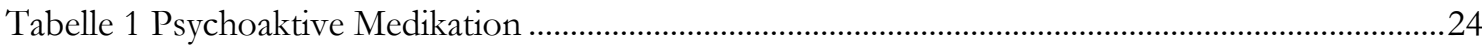

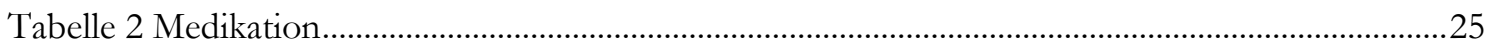

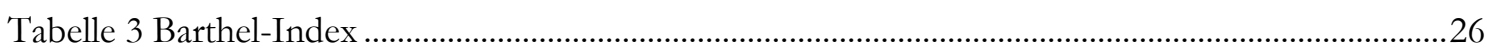

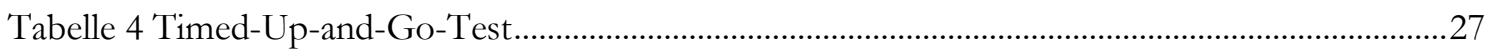

Tabelle 5 Tinetti-Test/POMA Performance-Orientated Mobility Assessment....................................27

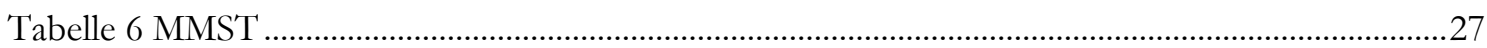

Tabelle 7 Sturzhäufigkeit in den Sturzjahren 2013 und 2014 ..............................................................32

Tabelle 8 Sturzhäufigkeiten in den behandelnden Abteilungen 2013 und 2014 ..................................33

Tabelle 9 Stichprobe nach Geschlechtszugehörigkeit......................................................................

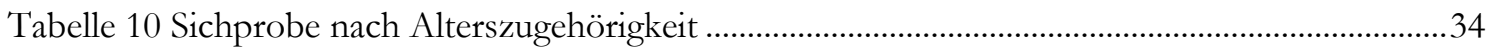

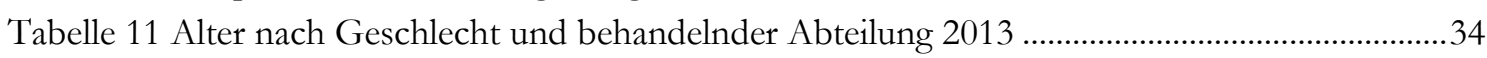

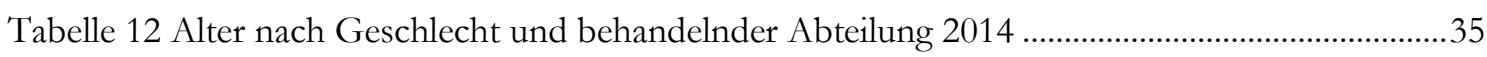

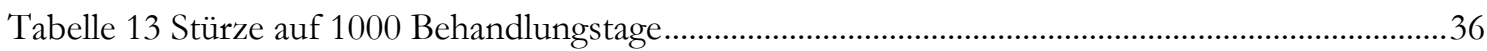

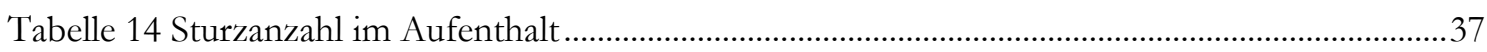

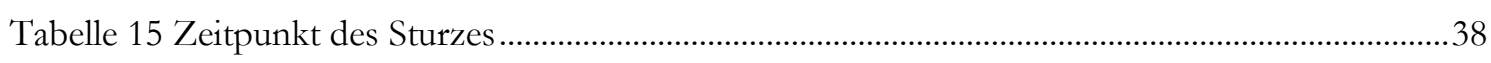

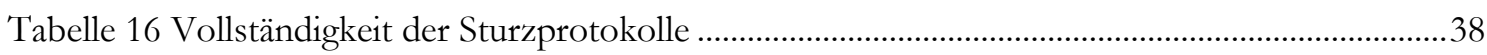

Tabelle 17 Zeitpunkt des Sturzes und Vollständigkeit der Sturzprotokolle............................................39

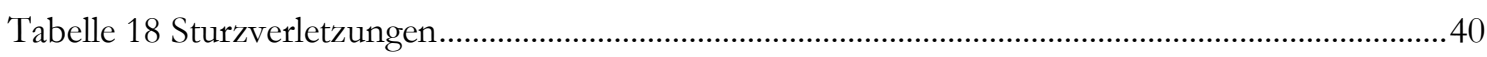

Tabelle 19 Mittelwertunterschiede Sturzjahr 2013 und Kontrollen 2013 ...........................................41

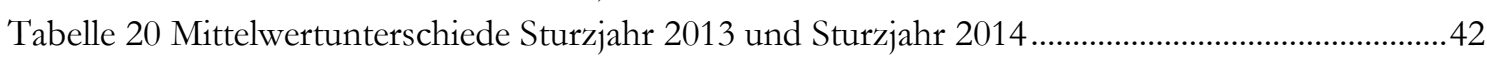

Tabelle 21 Einfluss psychoaktiver Medikamente auf das Sturzrisiko ........................................................ 43

Tabelle 22 Einfluss nicht psychoaktiver Medikamente auf das Sturzrisiko ............................................4

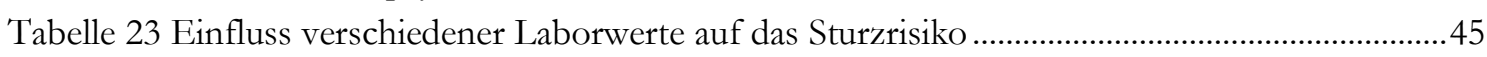

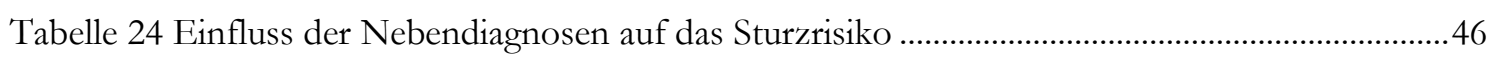

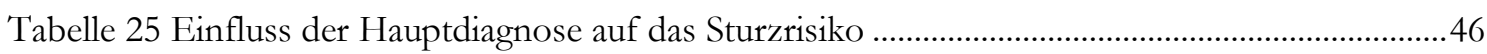

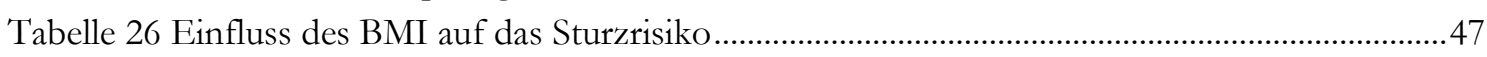

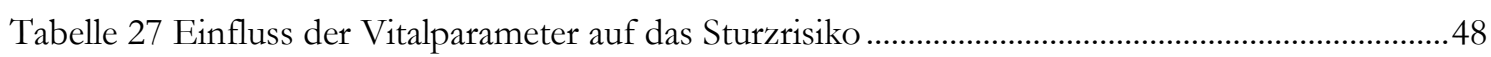

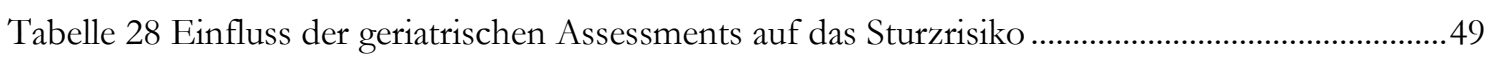

Tabelle 29 Anzahl eingenommener Psychopharmaka im Jahr 2013 .......................................................

Tabelle 30 Benzodiazepin-Dosis als Diazepam- ̈̈quivalente …………………………........................... 51

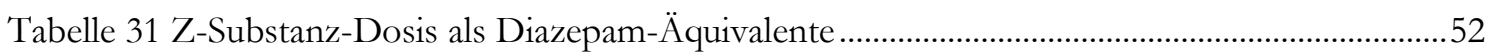

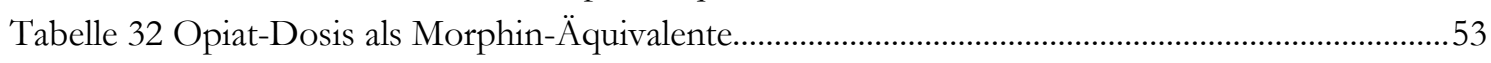

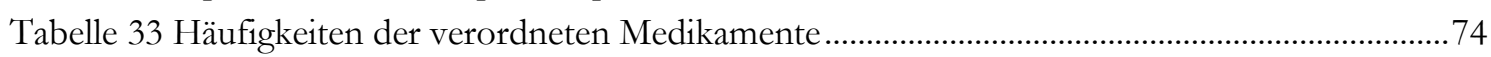

Tabelle 34 Kombinationen eingenommener Psychopharmaka Sturzjahr 2013 ......................................

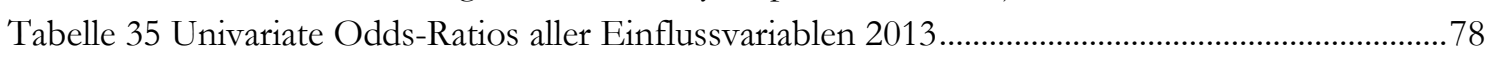

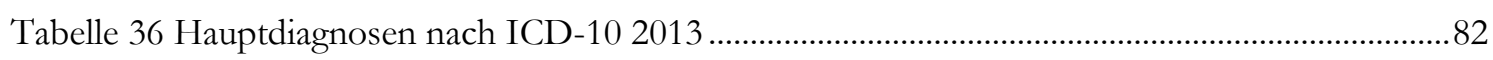

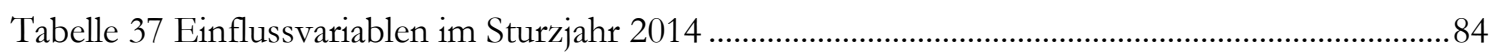

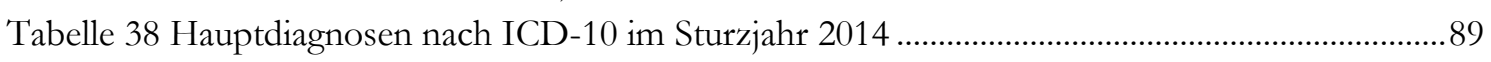

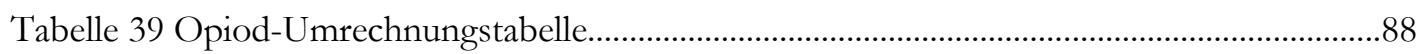




\section{Abkürzungsverzeichnis}

\begin{tabular}{|c|c|}
\hline $\mathrm{AD}$ & Antidepressivum/Antidepressiva \\
\hline Art. & arterieller \\
\hline ASR & Achillessehnenreflex \\
\hline $\mathrm{Bt}$ & Behandlungstage \\
\hline $\mathrm{DM}$ & Diabetes mellitus \\
\hline EKW & Evangelisches Krankenhaus Göttingen-Weende \\
\hline $\mathrm{KI}$ & Konfidenzintervall \\
\hline KHK & koronare Herzkrankheit \\
\hline MW & Mittelwert \\
\hline M. Parkinson & Morbus Parkinson \\
\hline MMST & Mini-Mental-Status Test \\
\hline $\mathrm{N}$ & Anzahl \\
\hline OR & Odds-Ratio \\
\hline PIM & potenziell inadäquate(s) Medikament(e) \\
\hline PSR & Patellarsehnenreflex \\
\hline SD & Standardabweichung \\
\hline SNRI & selektive Serotonin-Noradrenalin-Wiederaufnahmehemmer \\
\hline SSRI & selektive Serotonin-Wiederaufnahmehemmer \\
\hline TetraAD & tetrazyklische Antidepressiva \\
\hline TZA & trizyklische Antidepressiva \\
\hline UAW & unerwünschte Arzneimittelnebenwirkungen \\
\hline ZNS & zentrales Nervensystem \\
\hline
\end{tabular}




\section{Einleitung}

\subsection{Epidemiologische Daten}

Die Schenkelhalsfraktur ist mit einer Inzidenz von etwa 100.000 Frakturen pro Jahr oder 140 Frakturen pro 100.000 Einwohner die häufigste schwerwiegende Sturzverletzung des alten Menschen (Icks et al. 2008). Bei älteren Menschen ist der Sturz auf die Hüfte im häuslichen Umfeld die häufigste Ursache für einen Schenkelhalsbruch. Es wird angenommen, dass ca. 30\% aller über 65-Jährigen mindestens einmal im Jahr stürzen (Pasquetti et al. 2014). Bei den 80 - 89-Jährigen liegt die Häufigkeit sogar bei 40-50\% (Gulich 2008). 6070\% der gestürzten Patienten stürzen in den folgenden 12 Monaten erneut (Donald und Bulpitt 1999).

Laut des jährlichen Unfallberichts des Robert Koch-Instituts (RKI) machen Stürze bei Frauen 38\% aller nicht tödlichen Unfälle im häuslichen Umfeld aus. Sie sind damit deutlich häufiger betroffen als Männer mit 24,8\% (Lahmann et al. 2014).

Stürze älterer Menschen im häuslichen Umfeld sind ein in der Gesellschaft gut bekanntes und beachtetes Problem. Dass ältere Menschen gerade in einem scheinbar sicheren Umfeld wie dem Krankenhaus besonders gefährdet sind zu stürzen, ist hingegen weniger bekannt (Rubenstein 2006). Das Vorhandensein von Multimorbidität, Neuansetzung oder Umstellung von Medikamenten und die für den Patienten ungewohnte Umgebung führen zu einer hohen Prävalenz von Stürzen im Krankenhaus (Evans et al. 2001; Oliver et al. 2004; Rubenstein 2006).

Gerade im Hinblick auf die alternde Bevölkerung wird die Bedeutung von Stürzen und den damit einhergehenden Folgen noch weiter zunehmen. Mit fortschreitendem Alter nimmt die Multimorbidität zu, und der Grad an Pflegebedürftigkeit steigt (Miller et al. 2000; Neubart 2015). Jeder geriatrische Patient weist eine individuelle Problemkonstellation auf, die einen ganzheitlichen Ansatz in der Behandlung erfordert (Marik 2006). Neben der mit zunehmendem Alter eingeschränkten allgemeinen Gesundheit zeigt sich oft eine abnehmende kognitive Leistungs- und Anpassungsfähigkeit mit häufigem Auftreten von dementiellen Syndromen und damit einhergehend auch eine zunehmende Mobilitätseinschränkung (Tinetti 1986; Graham et al. 1997). So kommt es häufig zu Problemen in der Alltagsbewältigung und möglicherweise zum Verlust der Selbstständigkeit mit Aufgabe des eigenen 
Wohnraums und Umzug ins Pflegeheim (van Rensbergen und Nawrot 2010). Bestehende chronisch orthopädische, internistische oder neurologische Erkrankungen und die damit einhergehende Polymedikation erfordern große Anstrengungen in der Behandlung (Tinetti 2003; Nobili et al. 2011). Zusätzlich zum oft protrahierten Krankheits- bzw. Genesungsverlaufs treten häufiger Depressionen, Schlafstörungen und Unruhe- und Erregungszustände auf (Schwab 1992; Sass et al. 2010).

\subsection{Die Sturzhäufigkeit}

Es existiert eine Vielzahl von epidemiologische Studien zur Prävalenz und Inzidenz von Stürzen, die abhängig vom Erhebungsort und untersuchtem Patientenkollektiv unterschiedliche Ergebnisse liefern. Durch die oft länderspezifischen Unterschiede in der Betreuung alter Menschen und die in den verschiedenen Studien unterschiedlich angewandten Sturzbegriffe sind die Ergebnisse nicht immer vollständig vergleichbar (Hauer et al. 2006).

Es existieren zwei anerkannte Definitionen für Stürze, die in vielen Studien Verwendung finden. Das ProFaNE (Prevention of Falls Network Europe) definiert Stürze als ein „,[...] unerwartetes Event, bei dem der Körper von einem höheren zu einem niedrigeren Niveau, also dem Boden oder der Erde zu liegen kommt“ (Lamb et al. 2005). Die Definition der Kollegg International Working Group ähnelt der der ProFaNE allerdings schließt sie Ursachen wie Bewusstseinsverlust, plötzliche Lähmungen, Schlaganfall oder epileptische Anfälle für Stürze aus (Kellogg International Work Group 1987).

Als Dokumentationsinstrument werden meistens Incident Reports eingesetzt, die nach einem Sturz von einem Arzt oder dem Pflegepersonal ausgefüllt werden und variable Informationen zum Sturzereignis enthalten (Shorr et al. 2008; Hignett et al. 2013).

Dabei muss zwischen der Sturzhäufigkeit, also der Anzahl von Stürzen bei einer definierten Zahl von Patienten, und der Sturzinzidenz im Krankenhaus als Anzahl der Stürze pro 1000 Behandlungstage $(\mathrm{Bt})$ unterschieden werden. Sturzraten variieren in den meisten Studien zwischen 1,6 und 17 Stürzen pro 1000 Bt im Krankenhaus. In einer der größten durchgeführten Studien in den Vereinigten Staaten mit 315.817 eingeschlossenen Stürzen ergab sich beispielsweise eine Sturzrate von insgesamt 3,56 Stürzen je 1000 Bt (Bouldin et al. 2013). 
Die höchsten Sturzraten von 9,1 bis zu 17 Stürzen pro 1000 Bt zeigten sich in den geriatrischen Abteilungen (Heinze et al. 2002; von Renteln-Kruse und Krause 2004; Hill et al. 2007; Kinne und Klewer 2016).

In einer vom RKI groß angelegten von 2006-2013 durchgeführten Studie zur Sturzhäufigkeit in deutschen Krankenhäusern und Pflegeheimen ergaben sich über den gesamten Zeitraum errechnete Sturzhäufigkeiten von 3,9 \% (95 \%-KI 3,6-4,2) in Krankenhäusern und 4,6 \% (95 \%-KI 4,3-4,9) in Pflegeheimen (Lahmann et al 2014).

Sturzraten sind in Institutionen wie dem Krankenhaus oder dem Pflegeheim bis zu dreimal höher als bei zu Hause lebenden Menschen (Rubenstein et al. 1990).

Unterschiede in Sturzraten bzw. Sturzhäufigkeiten bestehen auch zwischen den Geschlechtern. Dabei scheinen Männer etwas gefährdeter zu sein als Frauen (Hitcho et al. 2004 ; von Renteln-Kruse 2004; Kinne und Klewer 2016).

Unabhängig vom Geschlecht steigt die Sturzfrequenz mit zunehmendem Alter der Patienten an ( Heinze et al. 2002; Williams et al. 2007; Pasquetti et al. 2014; Lahmann et al. 2014).

Es scheint eine Häufung von Stürzen in der Nacht zu geben (Hitcho et al. 2004; Fischer et al. 2005; Krauss et al. 2007; Kinne und Klewer 2016).

Studien zur Sturzprävalenz in Pflegeheimen zeigen ähnliche Resultate, jedoch sollte aufgrund unterschiedlicher Umgebungsfaktoren und der vielfältigen Gründe für die Krankenhauseinweisung davon abgesehen werden, Ergebnisse von Studien zur Sturzprävalenz zwischen Krankenhaus und Pflegeheim zu vergleichen (Rubenstein et al. 1990; Vu et al. 2004; Becker und Rapp 2011).

\subsection{Die Ätiopathogenese von Stürzen}

Stürze sind meist multifaktorieller Genese und Folge eines Zusammenspiels vieler verschiedener Faktoren. Es existieren verschiedene Einteilungen der Sturzursachen und Sturzmechanismen. Oft wird dabei zwischen extrinsischen und intrinsischen Ursachen unterschieden (Bueno-Cavanillas et al. 2000; Pasquetti et al. 2014):

Bei den extrinsischen Ursachen liegt ein Einfluss von außen auf den Sturzvorgang vor. Beispielsweise können herumliegende Gegenstände, bewegliche Nachtschränke oder nicht festgestellte Rollatoren den Patienten zum Stolpern oder Ausrutschen bringen. Diese Faktoren sind durch den Patienten nur bedingt beeinflussbar (Krauss et al. 2005; Pasquetti et 
al. 2014; Yang et al. 2017). Iatrogene Faktoren wie die vom behandelnden Arzt gewählte Medikation können auch zu den extrinsischen Ursachen gezählt werden (Morse et al. 1989a; Frels et al. 2002; Morse und Faan 2008).

Intrinsische Ursachen liegen im Patienten begründet. Es wird dabei zwischen den „normalen“ mit dem Alterungsprozess einhergehenden und den durch Krankheit hervorgerufene Faktoren unterschieden.

Die häufigste intrinsische Ursache stellen Probleme in der Mobilität, also bei Gang-und Standsicherheit dar (Sartini et al. 2010; Segev-Jacubovski et al. 2011). Außerdem kam die durch dementielle Syndrome induzierte Non-Compliance mit dem Vergessen von getroffenen Absprachen und dadurch risikobehaftetes Verhalten Auswirkungen auf das Sturzrisiko haben (van Doorn et al. 2003; Rubenstein 2006).

\subsection{Die Sturzrisikofaktoren}

Es kann davon ausgegangen werden, dass eine steigende Anzahl an Risikofaktoren mit dem Sturzrisiko positiv korreliert (Tinetti et al. 1988; Williams et al. 2007). Einige Risikofaktoren gelten nur für bestimmte Orte und Situationen, daher sind Studien zum Einfluss verschiedener Variablen auf das Sturzrisiko zwischen Krankenhaus und Pflegeheim nur bedingt miteinander vergleichbar. Im Folgenden werden die einzelnen Faktoren, die Einfluss auf das Risiko zu Stürzen haben, vorgestellt.

\subsubsection{Kognitive Fähigkeiten}

Da das Sturzrisiko mit steigendem Alter zunimmt, könnte man annehmen, dass das Alter ein wichtiger Risikofaktor für Stürze ist. Jedoch ist der biologische Prozess des Alterns mit einer Abnahme der kognitiven Fähigkeiten und Mobilität vergesellschaftet, sodass man eher von einem indirekten Effekt durch das Alter sprechen sollte (Miller et al. 2000).

Ein Effekt, der mit zunehmendem Alter bei vielen Patienten auftritt, ist die Abnahme der kognitiven Fähigkeiten. Als Messinstrumente der kognitiven Fähigkeiten wird vor allem der MMST eingesetzt (Folstein et al. 1975; Tombaugh und McIntyre 1992).

Häufig finden sich Diagnosen wie Demenz und Parkinsonsyndrom, unabhängig vom jeweiligen Subtyp, bei Sturzpatienten (van Doorn et al. 2003; Hill et al. 2007; Muir et al. 2012). 
Muir et al. ermittelten in ihrer Übersichtsarbeit, dass ein eingeschränkter kognitiver Status mit Ergebnissen im MMST von unter 27 Punkten mit einem erhöhten Sturzrisiko und daraus resultierenden Verletzungen assoziiert war (Muir et al. 2012).

Wichtige Symptome, die gehäuft bei Patienten mit eingeschränktem kognitivem Status auftreten, sind reduziertes Erinnerungsvermögen, Probleme bei der Orientierung und Verwirrtheitszustände (Herman et al. 2010).

Dies führt dazu, dass Patienten möglicherweise uneinsichtig bezüglich ihrer eigenen körperlichen Fähigkeiten werden und Absprachen zwischen Patient und Pflegepersonal eventuell nicht verstanden werden können (Lim et al. 2014).

Es gilt außerdem als gesichert, dass eine Abnahme der kognitiven Fähigkeiten mit einem langsameren Gangbild, unsicherem Stand und reduzierter Balance einhergeht, wodurch Stürze ebenfalls begünstigt werden (van Doorn et al. 2003; Segev-Jacubovski et al. 2011; Muir et al. 2012).

\subsubsection{Mobilität}

Eine eingeschränkte Bewegungsfähigkeit ist bereits vielfach als ein wichtiger Risikofaktor für Stürze identifiziert worden (Tinetti 1986; Rubenstein 2006; Kegelmeyer et al. 2007; McMichael et al. 2008; Müller et al. 2009).

Mobilität umfasst sowohl Balance im Stand als auch Gangbild und Muskelkraft. Stel et al. ermittelten beispielsweise ein Odds-Ratio (OR) von 2,8 für Einschränkungen im aufrechten Stand als größten Risikofaktor für Stürze innerhalb eines Jahrs (Stel et al. 2003).

Es existieren eine Vielzahl von Mobilitätstests in der klinischen Praxis. Timed-Up-and-Go, Romberg-Versuch und der Tinetti-Mobilitätstest (heute auch Performance-Orientated Mobility Assessment, kurz „POMA“ genannt) sind wohl die geläufigsten Tests und werden auch in der hausärztlichen Medizin zur Verlaufskontrolle angewandt, um dem Patienten bei Zunahme der Einschränkungen rechtzeitig Hilfe zukommen zu lassen (Nikolaus 2001).

Im stationären Bereich gelten der Timed-Up-and-Go-Test sowie der Tinetti-Mobilitätstest als verlässliche Werkzeuge zur Vorhersage der Sturzgefährdung aufgrund von Mobilitätsdefiziten (Tinetti 1986; Beauchet et al. 2011).

\subsubsection{Multimorbidität}

Multimorbidität wird definiert als das Vorhandensein von zwei oder mehr chronischen Erkrankungen bei einem Patienten (Divo et al. 2014). Sie steht im Zusammenhang mit 
verminderter Lebensqualität, funktionellen Einschränkungen und vermehrter Inanspruchnahme medizinischer Einrichtungen (Marengoni et al. 2011; Wallace et al. 2015). Multimorbidität im Alter ist häufig. In einer großangelegten Studie der Gmünder Ersatzkasse (heute Barmer -Ersatzkasse) wiesen 62\% aller über 65-jährigen Versicherten mehr als drei chronische Erkrankungen auf (van den Bussche et al. 2011).

Der multimorbide geriatrische Patient erfordert große Anstrengungen bei der Auswahl einer angemessenen Therapie. Dabei kann es zu Konflikten innerhalb der einzelnen behandelnden Fachrichtungen kommen, da eine leitliniengetreue Behandlung nicht immer gewährleistet ist (Markun et al. 2014). Multimorbidität wird auch als direkter Risikofaktor für Stürze angesehen (Battegay et al. 2017).

Einzelne Diagnosen wie Morbus Parkinson, Hirninfarkte und Demenz sind bereits mehrfach mit Stürzen in Verbindung gebracht worden (Salgado et al. 1994; Morley 2007; Jansen et al. 2015).

Hill et al. untersuchten als erste Arbeitsgruppe auf Basis von DRG, welche Diagnosen das Sturzrisiko erhöhen. Dabei zeigten Demenz, Delirium, Schlaganfall und respiratorische Infektionen signifikante Assoziation mit Stürzen (Hill et al. 2007).

Insbesondere demente Patienten neigen dazu, Verwirrtheitszustände (Delirien) im stationären Aufenthalt zu entwickeln (Gogol 2008; Fong et al.2009; Mazur et al. 2016). Funktionelle Einschränkungen und der Verlust von Unabhängigkeit werden als wichtige Komponenten von deliranten Zuständen genannt (Fong et al. 2009). Zur pharmakologischen Therapie eines Delirs werden Neuroleptika und Benzodiazepine empfohlen (Lorenzl et al. 2012). In Fall-Kontrollstudien zeigten delirante Patienten oft die höchsten Sturzwahrscheinlichkeiten (Oliver et al. 1997; Hendrich et al. 2003).

Allerdings gilt es dabei auch zu bedenken, dass viele der genannten Diagnosen eine Behandlung mit Medikamenten erfordern, die ebenfalls das Risiko für Stürze erhöhen. Die Berechnung des Risikos für einzelne Diagnosen und Medikamente erfordert dann komplexe statistische Methoden, auf die später in Kapitel 3.6.2 dieser Arbeit eingegangen wird.

\subsubsection{Medikation}

Im Krankenhaus verabreichte Medikamente zählen zu den iatrogenen Faktoren, die sich auf das Sturzrisiko auswirken können. Insbesondere Medikamente, die ihre Wirkung im zentralen Nervensystem entfalten, stehen schon lange im Verdacht, das Sturzrisiko zu erhöhen (Leipzig et al. 1999a). 
Es existieren 4 bedeutende Übersichtsarbeiten, die nahezu alle Studien einschließen, die Sturzrisikofaktoren bis zum Jahr 2015 untersuchten. Leipzig et al. (1999 a) schlossen als erstes Literaturreview 43 Studien, die Risikofaktoren in Pflegeheimen, Krankenhäusern und im häuslichen Umfeld untersuchten, in ihre Studie ein. Hartikainen et al. 2007; Woolcott et al. 2009 und Park et al. 2015 griffen diese Ergebnisse auf und komplettierten die Resultate durch neuere Studien zu bisher nicht untersuchten Medikamentenklassen. Allerdings werden dabei vor allem Studien in Pflegeheimen und im häuslichem Rahmen repräsentiert. Studien zu Sturzrisikofaktoren im Krankenhaus sind bei Leipzig et al. nur mit fünf untersuchten Studien vertreten. Ähnlich verhält es sich bei den anderen Übersichtsarbeiten. Bei den verwendeten Studiendesigns handelt es sich meist um Fall-Kontroll-Studien, CaseCrossover-Studien oder prospektive Kohortenstudien. Es wird nur eine randomisierte kontrollierte Studie beschrieben (Katz et al. 2004).

Im Fokus der Untersuchungen stehen dabei besonders die psychoaktiven Medikamentengruppen. Dazu zählen: Benzodiazepine, Neuroleptika, Antidepressiva, Z-Substanzen und Antikonvulsiva (Böhm 2016).

Psychoaktive Medikamente können unerwünschte Effekte wie eingeschränkter Reaktionsfähigkeit, Somnolenz, Agitation, orthostatische Dysregulation, reduzierte Akkommodationsfähigkeit und Kraftlosigkeit hervorrufen (Lavan und Gallagher 2016). Gerade ältere Menschen sind für solche adversen Effekte besonders vulnerabel. Dies liegt unter anderem an Veränderungen bei der Pharmakokinetik und Pharmakodynamik der Medikamente. Insbesondere eine verlangsamte Ausscheidung bei eingeschränkte Nierenfunktion ist bei älteren Patienten zu berücksichtigen (Mangoni und Jackson 2004).

Für Benzodiazepine wurde in vielen Studien ein erhöhtes Risiko für Stürze nachgewiesen (Oliver et al. 1997; Passaro et al. 2000; Frels et al. 2002; Krauss et al. 2005; Ackroyd-Stolarz et al. 2009; Chang et al. 2011). Die Datenlage zu den verschiedenen Präparaten mit unterschiedlichen Halbwertszeiten und deren Sturzrisiko ist allerdings inkonsistent (Leipzig et al. 1999a; Passaro et al. 2000; Wang et al. 2001b; Leipzig et al. 1999a; Hartikainen et al. 2007; Walker et al. 2005; Passaro et al. 2000; Hartikainen et al. 2007). Sowohl lang-als auch kurzwirksame Präparate wurden mit Stürzen assoziiert (Leipzig et al. 1999a; Passaro et al. 2000; Hartikainen et al. 2007). Höhere Dosen zeigten sich im Vergleich zu geringeren Dosen als risikoreicher (Wang et al. 2001a; Cumming und Le Couteur 2003; Blachman et al. 2017). Reidenberg et al. konnten bereits 1978 zeigen, dass die Plasmakonzentration nicht nur von der verabreichten Dosis, sondern auch vom Alter der behandelten Patienten abhing (Reidenberg et al. 1978). 
Darüber hinaus birgt die Verordnung von Benzodiazepinen und Opiaten auch ein Abhängigkeitspotenzial, mit den für die Patienten daraus resultierenden Problemen (Pétursson 1994; Llorente et al. 2000; Fernandez und Cassagne-Pinel 2001; Ashton 2005). Trotz der vielfältigen Probleme werden Benzodiazepine in niedriger Dosierung nach wie vor zur kurzfristigen Behandlung bei Schlafstörungen empfohlen (Leitlinie Nicht erholsamer Schlaf 2017).

Für Neuroleptika ist die Datenlage nicht ganz so eindeutig. Zwar wurden sowohl typische, als auch atypische Neuroleptika mit Stürzen in Verbindung gebracht, jedoch zeigten sie sich auch in Studien bei psychiatrischen Patienten als protektiver Faktor (Leipzig et al. 1999a; Hartikainen et al. 2007; Woolcott et al. 2009).

Zu den wichtigsten unerwünschten Nebenwirkungen zählen extrapyramidal motorische Störungen, die Stürze begünstigen könnten (Nissen et al. 2014). Allerdings wird auch die Hyponatriämie als unerwünschte Nebenwirkung genannt, die einen gesonderten Risikofaktor für Stürze darstellt (Renneboog et al. 2006; Moret et al. 2008; Gankam Kengne et al. 2008).

Niedrigpotente Neuroleptika werden aufgrund des etwas günstigeren Nebenwirkungsprofils und der schlafanstoßenden Wirkung im Krankenhaus gerne bei alten Patienten mit Schlafstörungen und Unruhezuständen eingesetzt (Nissen et al. 2014; Benkert und Steiger 2014; Carton et al. 2015).

Studien zum Sturzrisiko unter Neuroleptika- Dauereinnahme finden sich vor allem im Pflegeheim (Rigler et al. 2013). Studien zur Verordnung im Krankenhaus sind selten (Kallin et al. 2004; Tamiya et al. 2015).

Auch für Antidepressiva (TZA, SSRI, SNRI) ist bereits ein erhöhtes Risiko nachgewiesen worden (Chiu et al. 2015). Leider sind die neueren SNRI (Duloxetin wurde im Jahr 2004 zugelassen) bislang kaum untersucht (Woolcott et al. 2009; Quach et al. 2013; Park 2015). Auch unter der Behandlung mit SSRI und SNRI können vielfältige unerwünschte Nebenwirkungen auftreten (Ferguson 2001; Mandrioli et al. 2006; Letmaier et al. 2012). Zu Mirtazapin, dass als sogenanntes tetrazyklisches Antidepressivum gerade für ältere Menschen aufgrund der guten Verträglichkeit bei Schlafstörungen empfohlen wird, existieren bislang keine Studien zum Sturzrisiko in Krankenhäusern (Schatzberg et al. 2002; Holt et al. 2010; Alam et al. 2013; Henschel et al. 2015). Neuroleptika und Antidepressiva sind insbesondere auch wegen der vielen möglichen Arzneimittelinteraktionen bedenklich (Messiha 1993; Lane 1996; Richelson 1997; Spina und Scordo 2002; Urichuk et al. 2008; Kennedy et al. 2013). 
Auch die Anfang der 1990er Jahre zugelassen Z-Substanzen wurden bereits mit Stürzen assoziiert. Die Datenlage zu Stürzen im Krankenhaus ist jedoch dünn (Park et al. 2015). So wurde bislang für Zolpidem lediglich in zwei Studien ein erhöhtes Sturzrisiko nachgewiesen, für Zopiclon nur in einer Studie (Rhalimi et al. 2009; Cashin und Yang 2011; Chang et al. 2011).

Wang et al. untersuchten Zolpidem in einer großen Studie in der Langzeitanwendung im Pflegeheim und fanden unter der Einnahme von Zolpidem ein erhöhtes Risiko für Hüftfrakturen durch Stürze (Wang et al. 2001b). Zolpidem und Zopiclon galten lange aufgrund ihres im Vergleich zu den Benzodiazepinen geringeren Abhängigkeitspotenzials und der geringeren Belastung der Schlafarchitektur als Medikamente der Wahl bei Schlafstörungen im Alter (Darcourt et al. 1999; Holm und Goa 2000; Hajak et al. 2003). Jedoch wurde mittlerweile gezeigt, dass gerade das Abhängigkeitspotenzial vergleichbar mit dem von Benzodiazepinen ist (Victorri-Vigneau et al. 2007). Olubodun et al wiesen außerdem höhere Plasmakonzentrationen bei älteren Patienten im Vergleich zu jüngeren Patienten bei gleicher verabreichter Dosis nach (Olubodun et al. 2003).

Kallinn et al. 2004 konnten auch ein erhöhtes Sturzrisiko für Antikonvulsiva nachweisen, kontrollierten dabei allerdings nicht für Epilepsie als Confounder (Störfaktor) (Kallin et al. 2004).

Confounding by Indication stellt das Hauptproblem in den meisten Studiendesigns dar (Skelly et al. 2012). Gerade im stationären Rahmen treten häufig Symptome auf, die zum einen die Anwendung von psychoaktiven Medikamenten erfordern, zum anderen aber per se als Risikofaktoren für Stürze gelten. Zwar existieren statistische Methoden, die Confounding in ihre Risikoberechnung einbeziehen, jedoch ist es aufgrund der gezeigten multifaktoriellen Genese von Stürzen kaum möglich, alle Confounder in ein Modell einzubeziehen (Maldonado und Greenland 1993).

Auch nicht psychoaktive Medikamente wurden in Zusammenhang mit Stürzen untersucht. Allerdings existieren kaum Studien, die sich ausschließlich mit dem Risiko für NichtPsychopharmaka beschäftigen. Die Ergebnisse sind also meist ein Nebeneffekt der Suche nach Risikofaktoren für Stürze. Dies ist insofern problematisch, als in den Analysen mögliche Confounder nicht berücksichtigt werden (Leipzig et al. 1999b).

Leipzig et al. (1999 b) untersuchten in ihrer zweiten Übersichtsarbeit kardiale und schmerzreduzierende Medikamente auf ihr Sturzrisiko. Dabei konnte eine schwache Assoziation für Digoxin, Typ 1a-Antiarrythmika und Diuretika nachgewiesen werden. 
Allerdings zeigten andere Studien kontroverse Ergebnisse. In einer Studie fand sich beispielsweise ein protektiver Effekt für eine Diuretikatherapie (Frels et al. 2002). Allerdings wurde dabei nicht zwischen den verschiedenen Diuretikaklassen unterschieden. Denn gerade Thiaziddiuretika verursachen häufiger als Schleifendiuretika Hyponatriämien, die ebenfalls, wie bereits erwähnt, als ein Risikofaktor für Stürze gelten (Rodenburg et al. 2013).

Digoxin wurde auch in weiteren Studien als ein Risikofaktor für Stürze identifiziert (Gales und Menard 1995; Boutin et al. 2012). Gerade ältere Patienten scheinen für unerwünschte Nebenwirkungen wie Herzrhythmusstörungen durch Digitalis-Präparate besonders prädisponiert zu sein, da es aufgrund der häufig eingeschränkten Nierenfunktion zu Akkumulation mit Plasmakonzentrationsanstieg kommen kann (Currie et al. 2011).

Die Datenlage zu Antihypertensiva ist inkonsistent. Hartikainen et al. beschreiben in ihrer Übersichtsarbeit zwölf Studien, die eine Assoziation zwischen Beta-Blockern und peripheren Vasodilatatoren und Stürzen in Pflegeheimen herstellen. Vor allem zentral wirksame Alpha-2-Sympathikomimetika begünstigen eine orthostatische Hypotonie und werden daher auch nur noch bei therapierefraktärerer arterieller Hypertonie für ältere Menschen empfohlen (Lamy 1988; Judd und Calhoun 2012; Guideline (Leitlinie) Arterieller Bluthochdruck 2014). Beta-Blocker scheinen in neueren Studien hingegen eher einen protektiven Effekt hinsichtlich Stürzen zu haben (Gribbin et al. 2010; Park et al. 2015; Ham et al. 2017). Jedoch konnte auch gezeigt werden, dass vor allem die Einnahme mehrerer blutdrucksenkender Präparate das Risiko zu stürzen erhöht (Tinetti et al. 2014).

Auch Schmerzmedikamente wurden bereits auf ihr Sturz-förderndes Potenzial hin untersucht. Walker et al. fanden in ihrer Fall-Kontroll-Studie bei Patienten, die NSAR einnahmen, eine 10fach erhöhte Häufigkeit (OR 10,02) in ihrem Aufenthalt zu stürzen (Walker et al. 2005). Für Opioide wurde hingegen - trotz des sturzbegünstigenden Nebenwirkungsprofils (Schwindel, Mündigkeit) - ein eher protektiver Effekt in stationär durchgeführten Studien beobachtet (von Renteln-Kruse et al. 1998; Leipzig et al. 1999b; Walker et al. 2005; Prostran et al. 2016).

Vitamin- D-Substitution bei Vitamin D Mangel ist weit verbreitet und soll die Sturzhäufigkeit in Pflegeheimen sogar um bis zu 20\% reduzieren (Bischoff-Ferrari et al. 2009). Höhere Dosen (>800-1000IE/d) scheinen jedoch, ohne weitere pathophysiologische Erklärung, einen Risikofaktor für Stürze darzustellen (Sanders et al. 2010; Bischoff-Ferrari et al. 2016). 


\subsubsection{Polypharmazie}

Gerade die gleichzeitige Einnahme mehrerer Medikamente begünstigt unerwünschte Nebenwirkungen. Interaktionen, veränderte Wirkspiegel durch veränderte Resorption und Exkretion der Substanzen machen gerade alte multimorbide Patienten besonders anfällig für unerwünschte Arzneimittelwirkungen (UAW) (Sommeregger et al. 2010; Hammond und Wilson 2013; Blachman et al. 2017). Ca. 30\% der Krankenhausaufnahmen bei über 75Jährigen sind durch UAW bedingt (Runciman et al. 2003). In einer italienischen Studie nahm die Hälfte aller über 65-Jährigen aufgenommenen Patienten bereits mehr als 5 Medikamente ein (Nobili et al. 2011). Die Prävalenz für Medikamenten-Interaktionen aufgrund von Beeinflussung der hepatischen Cytochrom Enzyme betrug für 5 eingenommene Medikamente 80\% (Mallet et al. 2007). Ferner konnte gezeigt werden, dass sich die Risiken im Krankenhausaufenthalt zu stürzen, durch die Einnahme von mehr als 6 Medikamente verdreifacht (Chiu et al. 2015).

Aufgrund der verschiedenen Wechselwirkungen ist es nur schwer möglich, den Sturz nur einem Medikament zuzuordnen. Die aktuelle DEGAM Leitlinie zur Multimedikation sieht Stürze als ein Symptom für Arzneimittelunverträglichkeit (Leitlinie Multimedikation 2014). Insbesondere wird auch vor einer Art Verselbstständigung der Medikationsansetzung durch die sogenannte „Verschreibungskaskade“ gewarnt. Bei dieser Kaskade werden immer weitere Medikamente zur Therapie von Arzneimittelnebenwirkungen angesetzt (Rochon und Gurwitz 1997; Leitlinie Multimedikation 2014).

In einer Studie, die den Zusammenhang zwischen Schwindel und Stürzen untersuchte, zeigte sich, dass unter Schwindel leidende Patienten mehr Medikamente erhielten und öfter stürzten. Gerade die Kombination aus einem hypotensiv wirkendem Medikament mit einem Sedativum fand sich häufiger in der Schwindel- und Sturzgruppe (von Renteln-Kruse et al. 1998). Auch eine andere aktuelle Studie konnte eine Risikoerhöhung für sturzbedingte Frakturen durch Medikation mit mehr als 4 Medikamenten in Kombination mit einem Benzodiazepin nachweisen (Richardson et al. 2015).

Im Gegensatz zu den meisten Studien, die bereits gestürzte Patienten auf ihre Risikofaktoren hin untersuchen, konnte in einer Interventionsstudie durch systematisches Absetzen und Dosisreduktion von Medikamenten eine Reduktion des Sturzrisikos erreicht werden (van der Velde et al. 2007). 


\subsection{Sturzfolgen}

\subsubsection{Körperliche Folgen}

Die Einteilung der Sturzverletzungen erfolgt meist in drei Schweregrade: minimale, mittlere und schwere Verletzungen. Es wurde gezeigt, dass ca. 1/3 der Stürze mit Verletzungen einhergeht (Frels et al. 2002; Krauss et al. 2005; Berry und Miller 2008; Krauss et al. 2007; Lahmann et al. 2014). Schwere Verletzungen sind glücklicherweise mit Häufigkeiten von 411\% relativ selten. Zu den minimalen Verletzungen werden meist leichte Hautdefekte gezählt. Mittlere Verletzungen sind Platzwunden und Hämatome, und als schwere Verletzungen gelten Frakturen, Luxationen und Hirnblutungen (Hitcho et al. 2004; Berry und Miller 2008; Currie et al. 2011). Schwere Verletzungen scheinen dabei häufiger im Krankenhaus als im Pflegeheim oder im häuslichen Umfeld aufzutreten (Pasquetti et al. 2014; Lahmann et al. 2014).

\subsubsection{Psychische Folgen}

Neben den körperlichen Verletzungen leiden gestürzte Patienten häufig unter großer Angst vor weiteren Stürzen. In zwei Querschnittsstudien gab fast jeder zweite über 65-Jährige an, einmal Angst vor Stürzen und den Sturzfolgen gehabt zu haben (Zijlstra et al. 2007; Boyd und Stevens 2009).

Gestürzte Patienten sind für die Entwicklung des sogenannten Post-Sturz-Syndroms prädisponiert (Murphy und Isaacs 2004). Angst vor Stürzen führt dabei zu Reduktion von Mobilität und Aktivität, was wiederum zu verringerter Muskelkraft und Balancefähigkeit führt (Curcio et al. 2009). So entsteht ein Circulus Vitiosus, der zu wiederholten Sturzereignissen führen kann (Oliver et al. 2004; Morley 2007; Payette et al. 2016). Abnahme von Selbstständigkeit und Aufgabe des eigenen Zuhauses mit Umzug in ein Pflegeheim können die Folge sein (Zijlstra et al. 2007).

Frauen schienen in einer Studie dabei häufiger als Männer von Sturzängsten betroffen zu sein. Auch Parkinson-Patienten zeigten in dieser Studie öfter Angst vor Stürzen (Friedman et al. 2002).

Stürze in der Vorgeschichte stellen nicht nur einen sehr wichtigen Risikofaktor für weitere Stürze dar, sondern sind auch mit einer erhöhten Morbidität assoziiert (Oliver et al. 2000; Rubenstein 2006; Morley 2007) .

Nicht zuletzt wurde auch eine erhöhte Prävalenz von Depressionen unter Sturzpatienten gezeigt (Vetter und Ford 1989; Iaboni und Flint 2013). 
Im Krankenhaus gestürzte Patienten wiesen in vielen Studien eine verlängerte Aufenthaltsdauer auf (Krauss et al. 2007; Müller et al. 2009; Nobili et al. 2011; Dunne et al. 2014). Jedoch existieren keine Studien, die untersuchen, ob es sich bei der verlängerten Aufenthaltsdauer um einen eigenen Risikofaktor handelt oder um die Folge der höheren Morbidität bei Sturzpatienten.

\subsection{Behandlungskosten}

Nicht zuletzt sind Stürze auch ein gesundheitsökonomisches Problem. Verlängerte Liegedauern von Sturzpatienten steigern die Behandlungskosten und binden Ressourcen (Rizzo et al. 1998; Stevens et al. 2006; Morello et al. 2015). Die Kosten für die Behandlung von Sturzverletzungen variieren zwischen ca. 2000 und 42000 US-Dollar (Heinrich et al. 2010). Die Behandlung einer Schenkelhalsfraktur wird in Deutschland beispielsweise mit Summen zwischen 6410 und 6654 Euro vergütet (Frerichmann et al. 2007). Zahlen zur Gesamtbelastung der Gesundheitssysteme durch Stürze variieren länderabhängig. In den USA geht man von bis zu 19 Milliarden Dollar aus (Stevens et al. 2006). Morello et al. konnten in ihrer Studie zu den Behandlungskosten für im Krankenhaus gestürzte Patienten zeigen, dass sowohl verletzte, als auch nicht verletzte gestürzte Patienten signifikant höhere Behandlungskosten im Vergleich zu nicht gestürzten Patienten hatten (Morello et al. 2015). Darüber hinaus können Patienten, deren Angehörige oder der Medizinische Dienst der Krankenkasse Krankenhäusern oder Pflegeheimen bei Verletzungen durch Stürze eine Verletzung der Sorgfaltspflicht unterstellen und daraus Schadensersatzansprüche geltend machen. So können zusätzlich zu den höheren Behandlungskosten noch Kosten für Anwälte, Prozess und Schadensersatz anfallen (Schell 2009).

\subsection{Sturzprävention}

Es existieren verschiedene Ansätze in der Minimierung der Sturzprävalenz in Krankenhäusern, Pflegeheimen und dem häuslichen Umfeld. Zunächst gilt es als gesichert, das häusliche Umfeld bzw. die Patientenzimmer altersgerecht und sturzsicher zu gestalten. Dazu gehört beispielsweise, Flure mit Handläufen auszustatten, für adäquate Beleuchtung im Patientenzimmer zu sorgen oder rutschfeste Badematten in der Dusche zu installieren (Kellogg international work group 1987; Rubenstein 2006; Tzeng und Yin 2008). Neben der Beseitigung sturzgefährdender Umweltfaktoren ist eine Medikation zu wählen, die häu- 
fige Alterserscheinungen wie Multimorbidität und die veränderte Pharmakokinetik und dynamik berücksichtigt, um UAW zu verhindern. Aus diesem Grund sind Listen für potenziell inadäquate Medikamente (PIM) im Alter entwickelt worden (Holt et al. 2010). Zugeschnitten auf die Medikamente des deutschen Markts existiert die PRISCUS-Liste und für den amerikanischen Markt die Beers-Criteria. Es sind aber auch Listen auf Basis einer positiven Bewertung der Arzneimittel entwickelt worden. Für den deutschen Markt ist die FORTA-Klassifikation und für den britischen Markt sind die START/STOPP-Criteria zu nennen. Alle Listen entstanden durch Expertenkonsens (Barry et al. 2007; Laroche et al. 2009; Holt et al. 2010; Frohnhofen et al. 2011; Eckardt et al. 2014; Wickop und Langebrake 2014; Kuhn-Thiel et al. 2014; O'Mahony et al. 2015). In der klinischen Praxis zeigten sich je nach zu Grunde gelegter Liste erhebliche Unterschiede bezüglich der PIMAnwendungshäufigkeit (Siebert et al. 2013). Signifikante Unterschiede bezüglich unerwünschter Arzneimittelwirkungen fanden sich nicht (Wickop et al. 2016).

Um Risikopatienten früh zu identifizieren, wurden auf Basis der wichtigsten Risikofaktoren, sogenannte Risikoscores bzw. Risikoassessment-Tests entwickelt. Zu den am häufigsten genannten Risikofaktoren zählen dabei: Sturzvorgeschichte, Anzahl an Diagnosen (Multimorbidität), Stand und Balance und mentaler Status. Ähnliche Faktoren werden auch in vielen anderen Risikoassessments genannt (Oliver et al. 1997; Morse et al. 1989b; Lundin-Olsson et al. 2003). Viele Scores sind allerdings nicht validiert und liefern je nach Setting unterschiedlich gute Vorhersagewerte (Oliver et al. 2000; Campbell und Robertson 2007; Schwendimann et al. 2006a).

Neben den Sturzrisikoscores werden auch die geriatrischen Basisassessments zur Bestimmung des Sturzrisikos eingesetzt (Tinetti 1986; Podsiadlo und Richardson 1991; Ramirez et al. 2010; Mazur et al. 2016). Geriatrische Assessments sollten helfen klinische Zustände, Komorbiditäten und funktionelle Einschränkungen schnell zu erfassen, Therapien rechtzeitig einzuleiten und Hilfe bei der Alltagsbewältigung anzubieten (Rosen und Reuben 2011). Sie sind aufgrund geringer Sensitivität und niedriger positiv prädiktiver Werte zur Erfassung sturzgefährdeter Patienten eher weniger gut geeignet (Nikolaus 2001; Bouillon et al. 2013; Park 2018).

Der Tinetti-Test bzw. heute POMA-Test genannt ist ein rein motorischer Test, bei dem maximal 28 Punkte vergeben werden. 16 Punkte können dabei für Gleichgewicht und 12 Punkte in einer Gehprobe erreicht werden (Tinetti 1986). Es existieren jedoch viele Variationen des Tests, was seine Vergleichbarkeit erschwert (Köpke und Meyer 2006). Der Test zeigte in einigen Untersuchungen eine höhere Spezifität als Sensitivität und ist damit eher 
in der Lage, nicht sturzgefährdete Patienten zu identifizieren (Trueblood et al. 2001; Köpke und Meyer 2006; Kegelmeyer et al. 2007; Gates et al. 2008).

Der Barthel-Index (ADL-Score) umfasst die Partizipation am täglichen Leben. Dabei können in 10 Kategorien maximal 100 Punkte erreicht werden (Mahoney und Barthel 1965). Der Test gibt dabei Auskunft über die Mobilität und Selbstständigkeit einer Person. Niedrige Gesamtpunktzahlen im Test sind bereits mit Stürzen in Verbindung gesetzt worden (Krauss et al. 2005; Yokoya et al. 2007). Im Rahmen des Pflegeassessments bildet er ein wichtiges Element für die Einteilung der Pflegestufe.

Der MMST dient der Erfassung des kognitiven Status eines Patienten und findet Anwendung in der Demenzdiagnostik (Folstein et al. 1975; O'Bryant et al. 2008, Leitlinie Demenzen 2016). Da bereits gezeigt wurde, dass demente bzw. kognitiv eingeschränkte Patienten vermehrt gefährdet sind, zu stürzen, spielt auch das Abschneiden im MMST eine Rolle bei der Bewertung des Sturzrisikos (Graafmans et al. 1996; van Schoor et al. 2002). Die Grenzwerte für die Erhöhung des Sturzrisikos variieren zwischen 24 und 27 Punkten (Ramirez et al. 2010; Muir et al. 2012).

Der Timed-Up-and-Go-Test ist ein weiterer oft angewandter klinischer Test zur Bestimmung der Mobilität und des Sturzrisikos. Dabei wird der Patient gebeten, aus dem Sitzen aufzustehen, drei Meter zu gehen, sich umzudrehen und wieder zurück auf den Stuhl zu setzen (Podsiadlo und Richardson 1991; Barry et al. 2014). Die Zeitwerte, ab wann ein Patient dabei als sturzgefährdet gilt, variieren zwischen 10 und 32 Sekunden (Beauchet et al. 2011). Der Test hat, wie die anderen Assessments, keine hohe Sensitivität und Spezifität und ist daher nur bedingt zur Bestimmung des Sturzrisikos geeignet (Nocera et al. 2013; Barry et al. 2014). 


\section{Fragestellung und Ziel der Arbeit}

Welche Medikamente, Diagnosen und individuelle physische Parameter bei geriatrischen Patienten das Risiko, während des stationären Aufenthalts zu stürzen erhöhen, sind die Fragen, mit denen sich diese Arbeit auseinandersetzt. Dabei gilt es diese Risikoparameter herauszuarbeiten und daraus das Risikoprofil eines Sturzpatienten zu erstellen, um dann gegebenenfalls in naher Zukunft optimierte Sturzvermeidungsmaßnahmen zu ergreifen. Um die Zahl der gestürzten Patienten zu ermitteln, werden nach jedem Sturz im Evangelischen Krankenhaus Göttingen-Weende routinemäßig Sturzprotokolle angefertigt und im Rahmen des jährlichen Qualitätsberichts vom Medizincontrolling ausgewertet. Anhand dieser Sturzprotokolle sollen patientenbezogene Daten wie Diagnosen, Medikamente, Laborwerte und physische Parameter aus der Patientenakte ermittelt und die Umstände der Stürze untersucht werden.

So lassen sich folgende Fragestellungen der Arbeit ableiten:

1. Welche Medikamente stehen in einem Zusammenhang mit Stürzen?

2. Welche Diagnosen, Laborwerte und individuelle physische Parameter lassen sich bei Sturzpatienten finden und wie unterscheiden sie sich von denen der Patienten, die nicht stürzten?

3. Welchen Stellenwert haben die geriatrischen Assessments bei der Sturzprävention?

Außerdem soll der Frage nachgegangen werden, ob ein Zusammenhang zwischen der gleichzeitigen Einnahme mehrerer psychoaktiver Medikamente und dem Risiko, zu stürzen, besteht. Daran anschließend soll für einige Präparate versucht werden, eine Dosisabhängigkeit zum Sturzgeschehen herzustellen. 


\section{Patienten und Methoden}

\subsection{Studiendesign}

Zentrales Element dieser Dissertation stellt eine Fall-Kontroll-Studie dar. Die Fälle dieser retrospektiven Beobachtungsstudie bilden alle im Jahr 2013 gestürzten Patienten älter als 65 Jahre. Für die Kontrollgruppe wurden nicht gestürzte Patienten des 1. Quartals 2013, zugeordnet nach Alter, Geschlecht und Abteilungszugehörigkeit, ausgewählt und nach den gleichen Kriterien untersucht. Zusätzlich wurden alle Akten der Sturzpatienten des Jahres 2014 untersucht. Es erfolgte zunächst eine Auswertung aller Akten, die ein Sturzprotokoll enthielten. Grundlage der quantitativen Studie war ein Excel-Dokument, welches alle zu erfassenden Variablen enthielt. Die Akten wurden ausgehend von einer kurzen Analyse der Sturzprotokolle auf die weiter unten aufgeführten Parameter hin untersucht. Von essentieller Bedeutung waren dabei die Pflegekurven, die Informationen über die tägliche Medikation und Bedarfsmedikation enthielten. Diagnosen wurden dem Krankenhausinformationssystem Orbis sowie den Arztbriefen entnommen. Da die Medikation für Sturzpatienten bis $\mathrm{zu} 24 \mathrm{~h}$ vor dem Sturzereignis überprüft wurde, musste ein solcher Bezug auch für die Kontrollgruppe hergestellt werden. Als geeigneter Tag zur Erfassung der Medikation in der Kontrollgruppe wurde der Median der Tage bis zum Auftreten des Sturzereignisses in der Sturzgruppe gewählt, in dieser Studie der 5. Tag nach Aufnahme. Fehlende, das heißt nicht dokumentierte Werte, wie beispielsweise geriatrische Assessments in den nicht geriatrischen Abteilungen, konnten nicht ausgewertet werden.

\subsection{Stellenwert der Untersuchung im Gesamtprojekt}

Stürze werden schon lange als mögliche Komplikation in Zusammenhang mit der Gabe von Psychopharmaka, vor allem von Benzodiazepinen, vermutet und untersucht. Studien zum Sturzrisiko der neueren und alternativen Substanzen unter den Aspekten Dosisabhängigkeit und Altersbezug sind jedoch selten und wurden bisher zumeist im ambulanten Sektor, vor allem in Pflegeheimen, durchgeführt. Die Datenlage an deutschen Klinken ist bisher unbefriedigend (Modreker und von Renteln-Kruse 2009). Diese Dissertation ist Teil des Gesamtprojekts „,...da gab es so wunderbare Schlaftabletten - Verordnungen von Hypnotika und Sedativa an der Schnittstelle zwischen Krankenhaus und Hausarzt", das Psychopharmaka-Verordnungen im ambulanten und stationären Bereich mit quantitativen und qualitativen Methoden untersucht und ausgewertet hat. Durchgeführt wurde das Projekt vom Institut für Allgemeinmedizin der Universitätsmedizin Göttingen in Kooperation 
mit dem Evangelischen Krankenhaus Göttingen-Weende und dem Medizinischen Dienst der Krankenversicherung Mecklenburg-Vorpommern e.V. Gefördert wurde das Projekt durch das Bundesministerium für Gesundheit. Das Projekt gliedert sich in drei Phasen. Zunächst wurden in der 1. Phase Psychopharmaka-Verordnungen im Evangelischen Krankenhaus Göttingen-Weende bei Patienten $\geq 65$ Jahre untersucht. Dabei wurden Verschreibungshäufigkeiten von Psychopharmaka in Patientenakten erfasst. Parallel sollten Daten des Medizinischen Dienstes der Mecklenburg-Vorpommern e.V. zu PsychopharmakaVerordnungen vor und nach stationärem Aufenthalt ausgewertet werden. Zusätzlich wurde mittels Fragebögen und Interviews die persönliche Einstellung der Patienten, der Pflegekräfte und der behandelnden Ärzte zu Risiko und Nutzen bei Verschreibungen von Psychopharmaka erfasst, um die Gründe für die Verschreibungen zu evaluieren. Diese Dissertation kann gewissermaßen als eine tiefergehende Analyse der Untersuchungen der 1. Phase betrachtet werden, da sie Komplikationen unter der Therapie mit Psychopharmaka untersucht. In der 2. Phase sollten, ausgehend von den Ergebnissen der 1. Phase, geeignete Interventionen durch Fokusgruppen aus Pflegepersonal, niedergelassenen und am Krankenhaus tätigen Ärzten entwickelt werden, um die vorschnelle Verordnung von Hypnotika und Sedativa zu vermeiden. Die 3. Phase basiert schließlich auf den Ergebnissen aus Phase 1 und Phase 2 und soll die entwickelten Interventionen implementieren und evaluieren (Heinemann et al. 2016; Arnold et al. 2017).

Als wichtige Kooperationspartner dieses Projekts fungierten:

- Evangelisches Krankenhaus Göttingen-Weende:

- Frau Dr. med Claudia Choi-Jacobshagen (Chefärztin der Abteilung plastische, ästhetische und rekonstruktive Chirurgie),

- Prof. Dr. Michael Karaus (Chefarzt der Abteilung Innere Medizin),

- Dr. Joachim Dörges (Chefarzt der Abteilung Unfallchirurgie/Orthopädie),

- Prof. Dr. Klaus Langer (Chefarzt der Abteilung Allgemein-, Viszeral-, Thorax- und minimalinvasive Chirurgie),

- Prof. Dr. Hans-Werner Gottfried (Chefarzt der Abteilung Urologie und Kinderurologie),

- Prof. Dr. Detlef Brehmer (Chefarzt der Abteilung HNO),

- Olaf Hußmann (Direktor des Pflegedienstes)

- Institut für Allgemeinmedizin der Universität Göttingen:

- Prof. Dr. Wolfgang Himmel (Akademischer Oberrat) 


\subsection{Ort der Datenerhebung}

Die Datenerhebung erfolgte im Evangelischen Krankenhaus Göttingen-Weende, einem Krankenhaus der Grund- und Regelversorgung mit rund 485 Betten an den Standorten Göttingen-Weende und Bovenden-Lenglern. An beiden Standorten werden ca. 1100 Mitarbeiter beschäftigt, darunter 140 Ärzte und 440 Krankenschwestern und -pfleger. Das Krankenhaus verfügt über alle relevanten Fachabteilungen. Im Rahmen dieser Studie wurden folgende Fachbereiche des Standorts Göttingen-Weende berücksichtigt:

- Geriatrie,

- Innere Medizin,

- Unfallchirurgie/Orthopädie,

- Allgemein-, Viszeral-, Thorax- und minimalinvasive Chirurgie,

- Plastische, ästhetische und rekonstruktive Chirurgie,

- Urologie,

- Hals-, Nasen-, Ohrenheilkunde.

Für die Durchführung der Studie wurde angesichts der Thematik bewusst ein Krankenhaus der Grund- und Regelversorgung mit einer großen geriatrischen Abteilung mit insgesamt 96 Betten auswählt. Patientenakten sind im Evangelischen Krankenhaus GöttingenWeende in einem Zentralarchiv gelagert und somit schnell verfügbar. Die Sturzprotokolle als Dokumentationsinstrument im Evangelischen Krankenhaus Göttingen-Weende waren außerdem ein guter möglicher Zugang zu den Daten der Sturzpatienten. Die Zustimmung der jeweiligen Chefärzte der Abteilungen, die in die Studie aufgenommen wurden, erfolgte nach schriftlicher und mündlicher Projektvorstellung mittels anschließender Einverständniserklärung per Unterschrift.

\subsection{Patientenkollektiv}

Erfasst wurden alle Patienten, die in ihrem Krankenhausaufenthalt im Evangelischen Krankenhaus Göttingen-Weende vom 01.01.2013 bis zum 31.12.2014 stürzten und deren Sturz mittels Sturzprotokoll dokumentiert wurde.

In die Untersuchung eingenommen wurden Patienten mit einem Alter bei Aufnahme von 65 Jahren oder älter. Die Altersgrenze begründet sich darin, dass das Risiko zu stürzen, mit zunehmendem Alter steigt und auch in der Literatur häufig eine Grenze von 65 Jahren gewählt wurde (Tideiksaar 1996; Orimo et al. 2006; Homfeldt 2010). Da die Medikation anhand der PRISCUS-Liste in nach PRISCUS-Liste empfohlen und nicht empfohlen eingeteilt wurde und sich diese auf Patienten $>65$ Jahre bezieht, lag somit ein weiterer wichtiger Punkt für die Altersgrenze von 65 Jahren vor. Der Standort Bovenden-Lenglern konnte 
nicht mit in die Auswertung einbezogen werden, da die Akten vom Standort Göttingen aus nicht zugänglich waren. Für das Patientenkollektiv der Kontrollgruppe galten die gleichen Kriterien wie für das Sturzjahr 2013. Allerdings musste eine Mindestaufenthaltsdauer von fünf Tagen bestehen, damit die auf den Tag des Sturzes bezogenen Parameter auch für die Kontrollgruppe bestimmt werden konnten.

Somit ergaben sich zusammenfassend folgende Einschlusskriterien für die Sturzgruppe:

- Alter $\geq 65$ Jahre,

- Sturz im Evangelischen Krankenhaus Göttingen-Weende innerhalb des 01.01.2013 bis 31.12.2014.

Einschlusskriterien für die Kontrollgruppe:

- Alter $\geq 65$ Jahre,

- Aufenthalt von mindestens 5 Tagen und kein Sturz im Aufenthalt.

Die Ausschlusskriterien sahen dementsprechend wie folgt aus:

- Alter <65 Jahre,

- Aufnahme vor dem 01.01.2013 oder nach dem 31.12.2014,

- Patientenakte nicht verfügbar,

- Sturz während des Aufenthalts bei der Kontrollgruppe.

Da bei jedem Sturz ein Sturzprotokoll erstellt wird, kann ein Patient mehrere Sturzprotokolle auf sich vereinen. Ein Sturz meint dabei also immer einen Patienten und die zugehörige Patientenakte. Um Doppelerfassungen bei Abteilungswechseln, die auch mit einem Wechsel der Patientennummer einhergehen, im Aufenthalt zu vermeiden, musste bei jedem Patienten im Krankenhausinformationssystem Orbis kontrolliert werden, ob es gegebenenfalls bei einer Verlegung in eine andere Abteilung noch zu Stürzen gekommen ist. Für die Fall-Kontroll-Studie konnte nur ein Sturz pro Patient ausgewertet werden, da es sonst zu Doppeldokumentation einzelner Parameter gekommen wäre. Falls ein Patient in seinem Aufenthalt mehrfach gestürzt war, wurde dies dokumentiert und anschließend der Sturz mit den größten Sturzfolgen (vgl. 3.5.10) für die Fall-Kontroll-Studie ausgewählt. Trotz der häufigen Mehrfachstürze (21,73\% aller gestürzten Patienten stürzten mehr als einmal) konnten mit 481 Stürzen 2013 und 481 Stürzen 2014 in der Studie eine hohe Anzahl an Stürzen ausgewertet werden. Dass in beiden Jahren gleich viele Stürze ausgewertet wurden, ist Zufall. Bezogen auf die Gesamtzahl der Stürze von 805 im Jahr 2013 und 778 im Jahr 2014, die nicht zwischen dem Alter der gestürzten Patienten unterschieden und den Standort Bovenden-Lenglern mit einbezogen, konnte so ein großer Teil aller Stürze abgedeckt und erfasst werden. Für die Rekrutierung der Kontrollgruppe wurden Patienten aus dem 1. 
Quartal 2013 (01.01.2013-31.03.2013) ausgewählt, da für dieses Patientenkollektiv im Rahmen des Gesamtprojekts bereits ein Votum der Ethik-Kommission bestand.

\subsection{Datenerhebung}

\subsubsection{Fall-Kontroll-Studien}

Randomisierte kontrollierte Studien gelten bei klar definierter Fragestellung als Goldstandard in der medizinischen Forschung (Bothwell et al. 2016). Zur Erforschung von sturzassoziierten Risikofaktoren ist ein solches Studiendesign ethisch nicht vertretbar. Als nächstbeste Alternative bieten sich daher Fall-Kontroll-Studien an. Gut geplante und strukturierte Studien können dabei ebenfalls gute und sichere Ergebnisse liefern (Concato et al. 2000). Erkrankte Personen bilden dabei meist die Fallgruppe und gesunde Personen die Kontrollgruppe (Song und Chung 2010). In der hier vorliegenden Studie bilden gestürzte Patienten des Jahres 2013 die Fallgruppe und nicht gestürzte Patienten des 1. Quartals 2013 die Kontrollgruppe. Die Daten der Sturzpatienten des Jahres 2014 wurden als ergänzende Quelle zur Beschreibung des Risikoprofils eines Sturzpatienten herangezogen und bieten gegebenenfalls Potenzial für spätere Untersuchungen.

\subsubsection{Variablenauswahl}

Vor Beginn der Erhebung musste genau geplant werden, welche Parameter bei Sturzpatienten eine wichtige Bedeutung spielen können. Die Entwicklung einer fundierten ExcelMatrix mit vielen möglichen sturzassoziierten Variablen genoss somit in der Studienplanung größte Priorität. Orientiert an Literatur (Leipzig et al. 1999a, Leipzig et al. 1999b; Krauss et al. 2005; Hill et al. 2007; Park et al. 2015) und ergänzt um bisher nicht untersuchte Variablen wie geriatrische Basisassessments, Voroperationen, Polyneuropathie-typischeUntersuchungsbefunde und Vitalparameter am Sturztag konnten folgende Variablen in die Excel-Matrix aufgenommen werden:

- Behandelnde Abteilung (Innere Medizin, Geriatrie, Unfallchirurgie/Orthopädie, Urologie, Plastische Chirurgie, Hals-, Nasen-, Ohrenheilkunde, Allgemeinchirurgie),

- Alter,

- Geschlecht,

- Körpergröße,

- Körpergewicht,

- BMI,

- Anzahl der Stürze im Aufenthalt,

- Intervall zwischen Aufnahme und Tagen zum Sturz,

- Uhrzeit des Sturzes,

- Hauptdiagnose bei Entlassung nach ICD,

- Nebendiagnosen bei Entlassung: Art. Hypertonus, DM, KHK, Herzinsuffizienz, Demenz, Hirninfarkt, Parkinson Syndrom, Epilepsie, Exsikkose, Delir, 
- Polyneuropathie (PSR, ASR, Vibrationsempfinden),

- Voroperationen (untere Extremität, obere Extremität, Abdomen, Thorax, sonstige),

- Geriatrische Basisassessments (MMST, Barthel-Index, Tinetti-Test bzw. Demmi-Test, Timed-Up-and-Go),

- Medikation (Psychoaktive Medikation, Blutdruckmedikation, Antiarrhythmika, Opiate),

- Vitalparameter am Tag des Sturzes (Blutdruck, Herzfrequenz, Körpertemperatur),

- Laborwerte bei Aufnahme (Na, K, Ca, Kreatinin, C-reaktives Protein, Hämoglobin (Hb), Leukozyten),

- Sturzfolgen (leicht- und mittel, schwer),

- Vollständigkeit der Sturzprotokolle

\subsubsection{Datenerfassung}

Die aus den Patientenakten und dem Krankenhausinformationssystem Orbis entnommenen Daten wurden in eine viele Spalten umfassende Excel-Datenmatrix überführt. Arztbriefe dienten als Quelle für Nebendiagnosen, Barthel-Index, Tinetti-Test, MMST, TimedUp-and-Go-Test, Voroperationen und Sturzverletzungen. Hauptdiagnosen konnten aus den Abrechnungsunterlagen im System Orbis eingesehen werden. Die Laborausdrucke lieferte das Aufnahmelabor. Die Polyneuropathie-typischen Untersuchungsbefunde waren im Aufnahmeuntersuchungsbogen zu finden. Körpergröße und Gewicht, Bedarfsmedikation, Medikation am Sturztag und Vitalparameter konnten den Pflegekurven entnommen werden. Lag kein Eintrag für Körpergröße und Gewicht vor, konnten die Werte gelegentlich aus einem Ernährungsdokumentationsbogen erfasst werden. Die Medikation in der Abteilung Urologie musste auf einem separaten Medikationsbogen eingesehen werden. Die Sturzprotokolle lieferten Informationen über die Uhrzeit des Sturzes, Verletzungen und natürlich die Vollständigkeit der Protokolle. Die Anzahl der Stürze im Aufenthalt wurde dem Krankenhausinformationssystem Orbis entnommen.

\subsubsection{Einteilung der Nebendiagnosen}

Bei den Nebendiagnosen handelt es sich entweder um Diagnosen, die bereits vor dem Krankenhausaufenthalt bestanden oder auch um Diagnosen, die im stationären Aufenthalt zusätzlich zur Hauptdiagnose auftraten, beispielsweise ein postoperatives Delir. Die Einteilung der einzelnen Nebendiagnosen erfolgte als Diagnosegruppen. So fielen unter den Bereich KHK sowohl 1-,2- als auch 3-Gefäß-Erkrankungen. Diabetes mellitus umfasste alle Typen des Diabetes, die mit relativem- oder absolutem Insulinmangel assoziiert sind. In die Gruppe der Herzinsuffizienz wurden sowohl Rechts- als auch Linksherzinsuffizienz eingeordnet. M. Parkinson umfasste alle Parkinsonsyndrome, Hirninfarkt alle Formen der Ischämien im Gehirn. Der art. Hypertonus bezieht sich sowohl auf die primäre als auch die sekundäre Form. Gleiches galt für die Epilepsien. Die Diagnosen Exsikkose und Delir waren meist nicht näher spezifiziert. 


\subsubsection{Einteilung der psychoaktiven Medikation}

Psychoaktive Medikation oder Psychopharmaka meint im Folgenden immer ZNSwirksame Medikamente. Die Einteilung der Benzodiazepine erfolgte unter Berücksichtigung der verschiedenen Halbwertszeiten in Benzodiazepine mit kurzer-, mittellanger- sowie langer Wirkdauer (Böhm 2016). Neuroleptika wurden unter den Aspekten sedierend und antipsychotisch erfasst. Niedrigpotente Substanzen wirken vorwiegend sedierend und weniger antipsychotisch, hochpotente Substanzen vorwiegend antipsychotisch und weniger sedierend (Wang et al. 2005). Antidepressiva wurden unter pharmakologischen Aspekten in SSRI (selektive Serotonin-Wiederaufnahmehemmer), SNRI (Serotonin-NoradrenalinWiederaufnahmehemmer), trizyklische und tetrazyklische Antidepressiva zusammengefasst (Böhm 2016). Die verwendete Einteilung der verschiedenen Medikamente ist der Tabelle 1 zu entnehmen:

Tabelle 1 Psychoaktive Medikation

\begin{tabular}{|c|c|}
\hline \multicolumn{2}{|l|}{ Psychoaktive Medikamente } \\
\hline Kurz-und mittellang wirksame Benzodiazepine & SNRI \\
\hline Lorazepam & Duloxetin \\
\hline Lormetazepam & Venlafaxin \\
\hline Alprazolam & SSRI \\
\hline Brotizolam & Citalopram \\
\hline Temazepam & Escitalopram \\
\hline Midazolam & Fluoxetin \\
\hline Oxazepam & Paroxetin \\
\hline Langwirksame Benzodiazepine & Tetrazyklische Antidepressiva \\
\hline Bromazepam & Mirtazapin \\
\hline Nitrazepam & Maprotilin \\
\hline Tetrazepam & Trizyklische Antidepressiva \\
\hline Diazepam & Amitriptylin \\
\hline Flunitrazepam & Doxepin \\
\hline Dikaliumclorazepat & Trimipramin \\
\hline Clonazepam & Opipramol \\
\hline Neuroleptika antipsychotisch & Z-Substanz \\
\hline Quetiapin & Zopiclon \\
\hline Haloperidol & Zolpidem \\
\hline Risperidon & andere Sedativa \\
\hline Olanzapin & Baldrian \\
\hline
\end{tabular}




\begin{tabular}{|l|l|}
\hline Clozapin & Agomelatin \\
\hline Neuroleptika sedierend & Melatonin \\
\hline Melperon & Antikonvulsiva \\
\hline Prothipendyl & Pregabalin \\
\hline Promethazin & Gabapentin \\
\hline Neuroleptika andere & Valproinsäure \\
\hline Tiaprid & Lamotrigin \\
\hline & Carbamazepin \\
\hline & Levetiracetam \\
\hline & Oxcarbazepin \\
\hline
\end{tabular}

\subsubsection{Einteilung der weiteren Medikation}

Die weitere Medikation bzw. die Medikation mit nicht psychoaktiven Medikamenten bezieht sich auf die Einteilung der PRISCUS-Liste (s. Tabelle 2). Die einzelnen Medikamentengruppen wurden nach den Kriterien der PRISCUS-Liste als empfohlen bzw. nicht empfohlen eingeteilt.

Tabelle 2 Medikation

\begin{tabular}{|l|l|}
\hline \multicolumn{2}{|l|}{ Antihypertensive Medikation empfohlen } \\
\hline ACE-Hemmer/Sartane & Aldosteronrezeptor-Antagonisten \\
\hline Ramipril & Spironolacton \\
\hline Enalapril & Eplerenon \\
\hline Lisinopril & Alpha-2-Agonisten/Alpha-1-Antagonisten \\
\hline Candesartan & Moxonidin \\
\hline Eprosartan & Urapidil \\
\hline Irbesartan & Antihypertensiva nach PRISCUS-Liste nicht empfohlen \\
\hline Losartan & Doxazosin \\
\hline Olmesartan & Clonidin \\
\hline Telmisartan & Isosorbidmononitrat \\
\hline Valsartan & Nifedipin \\
\hline B-Blocker & Antiarrythmika \\
\hline Metoprolol & Antiarrhythmika nach PRISCUS-Liste empfohlen \\
\hline Bisoprolol & Digitoxin \\
\hline Carvedilol & Amiodaron \\
\hline Nebivolol & Diltiazem \\
\hline
\end{tabular}




\begin{tabular}{|l|l|}
\hline Atenolol & Verapamil \\
\hline Propanolol & Antiarrhythmika nach PRISCUS-Liste nicht empfohlen \\
\hline Sotalol & Digoxin \\
\hline Kalziumantagonisten & Opiate \\
\hline Lercanidipin & Buprenorphin \\
\hline Amlodipin & Tilidin \\
\hline Nitrendipin & Fentanyl \\
\hline Felodipin & Oxycodon \\
\hline & Hydromorphon \\
\hline & Morphin \\
\hline
\end{tabular}

Empfohlen und nicht empfohlen beziehen sich auf die PRISCUS-Liste, dunkelblau=Hauptgruppe, hellblau=Untergruppe

Zusätzlich wurde für jedes eingenommene Medikament die unmittelbar am Sturztag verabreichte Dosis erfasst. Für die sich anschließende Datenauswertung stand die verabreichte Medikamentendosis aber nicht im Vordergrund.

\subsubsection{Erfassung der Geriatrischen Assessments}

Die Einteilung der Geriatrischen Assessments ist den Tabellen 3, 4, 5 und 6 zu entnehmen: Anzumerken ist, dass für den Barthel-Index mehrere Interpretationsmöglichkeiten bestehen. Zum einen die pflegerische Bedürftigkeit (ADL-Score) und zum anderen die motorische Einschränkung (Mahoney und Barthel 1965, ICD-10 2013).

Tabelle 3 Barthel-Index

\begin{tabular}{|l|l|}
\hline \multicolumn{2}{|l|}{ Barthel-Index } \\
\hline $0-30$ & weitgehend pflegebedürftig \\
\hline $35-80$ & hilfsbedürftig \\
\hline $85-100$ & punktuell hilfsbedürftig \\
\hline
\end{tabular}

(Lübke et al. 2004) 
Tabelle 4 Timed-Up-and-Go-Test

\begin{tabular}{|l|l|}
\hline \multicolumn{2}{|l|}{ Timed-Up-and-Go-Test } \\
\hline$<10$ Sekunden & keine Mobilitätseinschränkung, schnell \\
\hline $11-19$ Sekunden & leichte Mobilitätseinschränkung, intermediate \\
\hline$>20$ Sekunden & relevante Mobilitätseinschränkung, langsam \\
\hline
\end{tabular}

(Podsiadlo und Richardson 1991)

Tabelle 5 Tinetti-Test/POMA Performance-Orientated Mobility Assessment

\section{Tinetti-Test}

\begin{tabular}{l|l}
$<20$ Punkte & erhöhtes Sturzrisiko
\end{tabular}

(Tinetti 1986, Faber et al. 2006)

Tabelle 6 MMST

\begin{tabular}{|l|l|}
\hline \multicolumn{2}{|l|}{ MMST } \\
\hline$<10$ Punkte & schwere Demenz \\
\hline 10-19 Punkte & moderate/mittelschwere Demenz \\
\hline 20-26 Punkte & leichte Demenz \\
\hline 27-30 Punkte & Keine Demenz \\
\hline
\end{tabular}

(Folstein et al. 1975; Deuschl und Maier 2009)

\subsubsection{Erfassung der Laborwerte}

Die Grenzwerte für die verschiedenen Labordiagnosen richten sich nach dem im Anhang beigefügten Laborausdruck.

\subsubsection{Erfassung der Vitalparameter}

- Blutdruck: $>140 / 90=$ Hypertonie, $<90 / 60=$ Hypotonie (Leitlinie Bluthochdruck 2014)

- Herzfrequenz: $>100 / \mathrm{min}=$ Tachykardie , $<60$ /min=Bradykardie (Cadenbach 2013, Stockburger und Möckel 2013)

- Körpertemperatur: normal $36,5-37,5{ }^{\circ} \mathrm{C}$, Temperatur $\geq 37,5^{\circ} \mathrm{C}$ (Dennhardt 2013) 


\subsubsection{Einteilung der Sturzverletzungen}

Orientiert an der Einteilung von Morse (2008) wurden die Verletzungen klassifiziert als:

- keine Verletzung

- leichte- und mittelschwere Verletzung

- schwere Verletzung

Leichte- und mittelschwere Verletzungen umfassten dabei Schürfwunden, Hämatome und Platzwunden. Bei schwere Verletzungen handelte es sich nur um Frakturen. Kombinationen wurden berücksichtigt und vermerkt.

\subsubsection{Erfassung der Medikation der Kontrollgruppe}

Da es bei der Kontrollgruppe keinen Sturztag zur Erhebung der Medikation gab, musste zu Vergleichbarkeitszwecken ein Stichtag ausgewählt werden, an dem die Medikation erfasst wurde (Walker et al. 2005, Chang et al. 2011). Da eine Erhebung der Medikation über einen längeren Zeitraum als 24 Stunden die erhaltene Medikation überschätzt, konnte ein Median der Variable „Intervall zwischen Aufnahme und Sturzereignis“ errechnet werden, der dann den 5. Tag nach Aufnahme als Stichtag der zu erfassenden Medikation in der Kontrollgruppe definierte.

\subsection{Datenauswertung}

Die Auswertung der Daten erfolgte mittels der Statistiksoftware SAS, Version 9.4. Die in der Excel-Datenmatrix erfassten Daten wurden in das Programm importiert und anschlieBend bearbeitet.

\subsubsection{Codierung}

Für die deskriptive Auswertung der Daten war es zunächst erforderlich, dichotome Variablen zu bilden und diese binär, also in 1 und $0 \mathrm{zu}$ codieren. So stand dann beispielsweise die Zahl 1=Ja für „Medikament erhalten“, $0=$ Nein für „Medikament nicht erhalten“.

\subsubsection{Statistische Auswertung}

Retrospektive Studiendesigns wie die Fall-Kontroll-Studie erfordern die Berechnung von Odds-Ratios zur Risikoabschätzung verschiedener Einflussvariablen. Ein Odds-Ratio ist gewissermaßen ein Maß für die Stärke eines Zusammenhangs zwischen zwei Variablen. Wichtig ist, dass man, wenn man von Odds spricht, Quoten meint und keine Wahrscheinlichkeiten. Odds-Ratios können dabei Werte von 0 bis unendlich annehmen, wobei ein Odds-Ratio von 1 ein gleich großes Quotenverhältnis bedeutet (Wirtz und Ulrich 2010). 
Um Odds-Ratios zu berechnen, gibt es verschiedene Methoden. Zunächst ist es wichtig sogenannte nicht adjustierte Odds zu berechnen. Diese zeigen den Einfluss von einer binären Variablen auf eine andere an und können in SAS mit dem Cochran-Mantel-HaenszelTest bzw. auch mit der proc logistic-Funktion berechnet werden. Um zu klären, wofür adjusted Odds errechnet werden, ist eine kurze Erläuterung des Themas Confounding (Störfaktor) notwendig.

Confounding ist in Fall-Kontroll Studien ein häufiges Problem (Maldonado und Greenland 1993). Der Begriff Confounding bedeutet die Beeinflussung einer Einflussvariable durch eine andere. So könnte die Nebendiagnose Delir bspw. die Einnahme eines Benzodiazepins oder Neuroleptikums erforderlich machen (Confounding by Indication) und so die unadjusted Odds von Benzodiazepinen oder Neuroleptika auf das Sturzereignis verfälschen. Um diesem Problem zu begegnen, wurden verschiedene Rechenalgorithmen entwickelt, die das Risiko von Confounding reduzieren (Christenfeld et al. 2004). In der Regel wird hierfür das Verfahren der multiplen logistischen Regressionsanalyse angewendet. Die Rechenvorschriften stepwise-, forward- und backward-Regression beziehen dann alle in das Modell eingefügten Variablen ein und errechnen Odds-Ratios, die für Confounding kontrolliert sind. Diese bezeichnet man dann als adjusted Odds.

Adjusted Odds wurden ebenfalls mittels der proc logistic-Funktion in SAS berechnet, jedoch bezog diese Form der Risikoberechnung die anderen Variablen mit ein.

Um eine optimale Regressionsanalyse durchzuführen, sind weitere Punkte zu beachten (Bagley et al. 2001). Zunächst gilt es, eine suffiziente Relation von Events zu Variablen einzuhalten, im Fall dieser Studie 481 Events auf 27 Einflussvariablen $=17,8$ Events pro Variable (Peduzzi et al. 1996). Dies ist vor allem wichtig, um eine optimale Berechnung der Regressionskoeffizienten zu gewährleisten (Babyak 2004).

Die beschriebene Thematik erforderte in dieser Studie eine Vortestung aller Variablen, um nicht zu viele Variablen in das Modell aufzunehmen. Diese Vortestung wurde mittels der Proc Freq-Funktion und dem Chi-Quadrat-Test bzw. Fisher's Exact Test für 4-Felder-Tafeln durchgeführt. Aufgenommen in das finale Regressionsmodell wurden dann alle Variablen, die das Signifikanzniveau von $\mathrm{p}=0,25$ unterschritten. Dieses sehr liberale Signifikanzniveau wurde gewählt, da vielfach gezeigt wurde, dass konventionelle p-Werte Variablen ausgeschlossen haben, die für das finale Modell von Bedeutung gewesen wären und so die Sensitivität der Modelle stark reduzierten (Shewart und Wilks 2005; Bursac et al. 2008).

Die dann signifikanten Variablen konnten in das finale Regressionsmodell überführt und mittels des stepwise-Verfahrens ausgewertet werden. Die Methode stepwise ist eine Kombination aus einer forward- und einer backward-Regression. Dabei wird zunächst eine Variable unter der Bedingung, dass sie das Modell optimiert, aufgenommen (Forward-Regression). Anschließend wird eine neue Variable eingefügt und getestet, ob sie das Modell verbessert. Geschieht dies nicht, wird sie entfernt (Backward-Elimination). Das Kriterium für das Verbleiben einer Variable im Modell war ein p-Wert von 0,1 (Bursac et al. 2008). Um zu über- 
prüfen, ob die anderen Verfahren gleichwertige Ergebnisse liefern, wurde zusätzlich eine Kontrollanalyse mittels einer bcackward-Regression durchgeführt. Einflussvariablen, die zu wenige Beobachtungen aufwiesen, konnten nicht in das Modell aufgenommen werden, da sonst die Sensitivität stark reduziert worden wäre.

An die Regression angeschlossen wurde der Hosmer-Lemeshow-Test als Goodnes of Fit-Test für das finale Modell durchgeführt. P-Werte nahe an 1 beschreiben dabei eine gute Anpassung des Modells an die Daten (Hosmer et al. 1997).

Die absoluten und relativen Häufigkeiten der einzelnen Einflussvariablen in den Sturzjahren 2013 und 2014 sowie in der Kontrollgruppe konnten mit der proc freq-Funktion berechnet werden.

Mittelwerte von kontinuierlichen Variablen wurden mit der proc means-Funktion errechnet. Daran angeschlossen erfolgte ein gepaarter Zweichstichproben-t-Test zur Bestimmung von Mittelwertunterschieden für Sturz- und Kontrollgruppe sowie ein ungepaarter Zweistichproben-t-Test für die beiden Sturzjahre 2013 und 2014 (Cressie und Whitford 1986). Für nicht kontinuierliche Variablen, wie die geriatrischen Assessments, wurden Verteilungsunterschiede mit dem Wilcoxon-Rangsummen-Test für gepaarte Stichproben auf statistische Signifikanz geprüft (Wilcoxon 1947).

Komplexere Kreuztabellen aus aggregierten Daten zur detaillierten Beschreibung der einzelnen Gruppen, das heißt Geschlechter, Alters und Abteilungsverteilung, konnten mit der proc-tabulate Funktion berechnet werden.

Darüber hinaus sollte versucht werden, einen Zusammenhang zwischen Sturz und Dosis einzelner Medikamente zu zeigen. So konnten für die Substanzen Benzodiazepine, ZSubstanzen und Opiode Äquivalenzdosen errechnet und dann mittels der proc freq-Funktion quantifiziert werden (Pereira et al. 2001, Ashton 2002). Eine Beispieltabelle der OpiodÄquivalenzdosen befindet sich im Anhang (s. Tabelle 39). Ob sich die Gruppen in den Häufigkeiten der verabreichten Dosen signifikant unterschieden, wurde mit dem WilcoxonRangsummen-Test überprüft (Lovric 2011). Als Maß für den Zusammenhang konnte der Kontingenzkoeffizient nach Pearson herangezogen werden (Tan et al. 2004). Außerdem sollte untersucht werden, ob ein Zusammenhang zwischen der Anzahl eingenommener psychoaktiver Medikamente und der Sturzhäufigkeit bestand. Dafür mussten die einzelnen Psychopharmaka-Variablen mittels der proc array-Funktion durchlaufen und aufsummiert werden. Eine Signifikanztestung erfolgte, wie bei den Äquivalenzdosen, mit dem Wilcoxon-Mann-Whitney-Test und als Zusammenhangsmaß wurde der Kontingenzkoeffizient nach Pearson angeführt.

Variablenimplementierungen im sogenannten Datastep wurden mit der proc print-Funktion überprüft. 


\subsection{Datenschutz und Genehmigung durch die Ethikkomission}

Vor Beginn der Datenerhebung erfolgte eine erneute Prüfung des um das Thema der vorliegenden Arbeit ergänzten Antrags durch die Ethikkommission der Universitätsmedizin Göttingen. Das Aktenzeichen lautete: 25/2/14.

Zur Wahrung des Datenschutzes wurden die Eingaben in die Datenmatrix auf einem geschützten Server der Universitätsmedizin Göttingen gespeichert. Die Patientendaten wurden pseudonymisiert und im Institut für Allgemeinmedizin der Universität Göttingen geschützt verwahrt. 


\section{Ergebnisse}

In diesem Kapitel erfolgt zunächst eine kurze Stichprobenbeschreibung, an die sich eine Betrachtung der Inzidenzraten von Stürzen sowie eine Prüfung der Sturzprotokolle auf Vollständigkeit anschließt. Daraufhin erfolgt eine detaillierte Darstellung der sturzverursachenden Risikofaktoren.

Zunächst wurden Mittelwertunterschiede einzelner Einflussvariablen in den Sturzjahren 2013 und 2014 sowie den Kontrollpatienten des Jahres 2013 betrachtet. Anschließend wurden die Verordnungshäufigkeiten der einzelnen Medikamente und die Häufigkeit anderer Risikofaktoren in der Sturzgruppe 2013 und in der Kontrollgruppe 2013 verglichen. Die geriatrischen Assessments wurden aufgrund fehlender Beobachtungen in anderen Abteilungen separat betrachtet. Abschließend wurden die verordneten Dosen von Benzodiazepinen, Z-Substanzen und Opiaten als errechnete Äquivalenzdosen in den Gruppen verglichen. Außerdem erfolgt eine Darstellung der summierten Anzahl eingenommener Psychopharmaka.

\subsection{Stichprobe}

Es wurden 1443 Patientenakten ausgewertet, die sich in die Gruppen Sturzjahr 2013 und Sturzjahr 2014, sowie die Kontrollgruppe 2013 aufteilten. Da nach Abteilung, Alter und Geschlecht zugeordnet wurde, sind die absoluten und relativen Häufigkeiten dieser Merkmale in der Gruppe der Stürze 2013 und der Kontrollgruppe 2013 identisch. Die Abteilung mit den meisten Stürzen war die Geriatrie, gefolgt von der Inneren Medizin und der Unfallchirurgie, sowohl im Jahr 2013 als auch im Jahr 2014 mit den meisten zu verzeichnenden Stürzen (s. Tabelle 7). Dies deckt sich mit den Daten des Medizincontrollings des Evanglischen Krankenhauses Göttingen-Weende (s. Tabelle 8).

Tabelle 7 Sturzhäufigkeit in den Sturzjahren 2013 und 2014

\begin{tabular}{|l|r|r|}
\hline \multicolumn{1}{|c|}{ Abteilung } & Sturzjahr 2013 & \multicolumn{2}{|c|}{ Sturzjahr 2014 } \\
\hline Geriatrie & 209 & 183 \\
\hline IM & 171 & 166 \\
\hline UCH & 59 & 58 \\
\hline ACH & 17 & 24 \\
\hline UR & 25 & 40 \\
\hline PCH & 0 & 9 \\
\hline HNO & 0 & 1 \\
\hline
\end{tabular}




\section{Summe}

481

481

*die Kontrollgruppe 2013 hat die gleiche Verteilung über die einzelnen Abteilungen wie im Sturzjahr 2013

Tabelle 8 Sturzhäufigkeiten in den behandelnden Abteilungen 2013 und 2014

\begin{tabular}{|l|r|r|}
\hline \multicolumn{1}{|c|}{ Fachabteilung } & Sturzjahr 2013 N (\%) & \multicolumn{1}{|c|}{ Sturzjahr 2014 N (\%) } \\
\hline Ohne Angabe & 0 & $1(0,1)$ \\
\hline PCH & $19(2,4)$ & $28(3,6)$ \\
\hline ACH & $17(2,1)$ & $18(2,3)$ \\
\hline URO & $25(3,1)$ & $43(5,5)$ \\
\hline UCH & $53(6,6)$ & $50(6,4)$ \\
\hline Geriatrie & $349(43,4)$ & $290(37,3)$ \\
\hline IM & $236(29,3)$ & $230(29,6)$ \\
\hline Pneu & $75(9,3)$ & $81(10,4)$ \\
\hline IMC & $16(2,0)$ & $15(1,9)$ \\
\hline Intensiv & $15(1,9)$ & $22(2,8)$ \\
\hline Summe & 805 & 778 \\
\hline
\end{tabular}

Daten des Medizincontrollings Evangelisches Krankenhaus Göttingen-Weende

Wie bereits in 4.3 erwähnt, wurden keine Stürze aus der Abteilung Pneumologie im Standort Lenglern ausgewertet. Außerdem wurden keine Stürze von Patienten jünger als 65 Jahre und pro Patient nur ein Sturz in die Auswertung mit einbezogen. So erklärt sich, wie es zu den Differenzen zwischen den Daten des Medizincontrollings und den Daten dieser Studie gekommen ist.

Der Anteil der weiblichen Patienten war in beiden Sturzjahren geringfügig größer als der Anteil männlicher Patienten, 58,8 \% (N=283) im Jahr 2013 und 52,8 \% (N=254) im Jahr 2014 (s. Tabelle 9).

Tabelle 9 Stichprobe nach Geschlechtszugehörigkeit

\begin{tabular}{|l|r|r|}
\hline Geschlecht & Sturzjahr 2013 N (\%)* & Sturzjahr 2014 N (\%) \\
\hline männlich & $198(41,2)$ & $227(47,2)$ \\
\hline weiblich & $283(58,8)$ & $254(52,8)$ \\
\hline Summe & 481 & 481 \\
\hline
\end{tabular}

*Kontrollgruppe 2013 hat die gleiche Verteilung 
Der Anteil der Patienten im Alter zwischen 75-85 Jahren war in beiden Sturzjahren am höchsten mit 42,8\% und 42\% (S. Tabelle 10). Der arithmetische Mittelwert für das Alter lag für das Sturzjahr 2013 bei 82,1 Jahren und für das Sturzjahr 2014 bei 82,0 Jahren (s. Tabelle 19 und Tabelle 20).

Tabelle 10 Stichprobe nach Alterszugehörigkeit

\begin{tabular}{|l|r|r|}
\hline \multicolumn{1}{|c|}{ Alter } & Sturzjahr 2013 N $(\%)^{*}$ & \multicolumn{1}{c|}{ Sturzjahr 2014 N (\%) } \\
\hline $65-74$ & $78(16,2)$ & $82(17,1)$ \\
\hline $75-85$ & $206(42,8)$ & $202(42,0)$ \\
\hline $85+$ & $197(40,1)$ & $197(41,0)$ \\
\hline Summe & 481 & 481 \\
\hline
\end{tabular}

*die Kontrollgruppe 2013 hat die gleiche Verteilung

Das höchste Durchschnittsalter der gestürzten weiblichen Patienten im Sturzjahr 2013 wies die Urologie mit 84,3 Jahren auf, allerdings mit nur 9 behandelten Patientinnen. Für die männlichen Patienten lag das höchste Durchschnittsalter bei 82,4 Jahren in der Allgemeinchirurgie, bei 8 behandelten Patienten. Die Mittelwerte für das Alter nach behandelnden Fachabteilungen und Geschlecht lagen zwischen 78,1 und 84,3 Jahren und waren damit relativ homogen verteilt (s. Tabelle 11).

Tabelle 11 Alter nach Geschlecht und behandelnder Abteilung 2013

\begin{tabular}{|c|c|c|c|c|c|c|c|c|}
\hline \multirow[b]{2}{*}{ Sturzjahr 2013* } & \multicolumn{8}{|c|}{ Alter } \\
\hline & & $\mathrm{N}$ & $\%$ & Mittelwert & Std & Median & Min & $\operatorname{Max}$ \\
\hline Geschlecht & Abteilung & & & & & & & \\
\hline \multirow[t]{5}{*}{ männlich } & Geriatrie & 82 & 17,0 & 81,2 & 6,71 & 82 & 69 & 94 \\
\hline & $\mathrm{IM}$ & 77 & 16,0 & 80,0 & 7,64 & 80 & 65 & 101 \\
\hline & $\mathrm{UCH}$ & 15 & 3,1 & 78,1 & 7,02 & 78 & 65 & 91 \\
\hline & $\mathrm{ACH}$ & 8 & 1,7 & 82,4 & 4,59 & 82,5 & 75 & 89 \\
\hline & UR & 16 & 3,3 & 79,5 & 6,1 & 79,5 & 68 & 88 \\
\hline \multirow[t]{5}{*}{ weiblich } & Geriatrie & 127 & 26,4 & 83,2 & 5,79 & 84 & 68 & 97 \\
\hline & IM & 94 & 19,5 & 84,2 & 7,57 & 86,5 & 65 & 98 \\
\hline & $\mathrm{UCH}$ & 44 & 9,2 & 81,9 & 7,7 & 82,5 & 65 & 89 \\
\hline & $\mathrm{ACH}$ & 9 & 1,9 & 79,8 & 5,04 & 79 & 72 & 96 \\
\hline & UR & 9 & 1,9 & 84,3 & 7,18 & 84 & 71 & 97 \\
\hline
\end{tabular}

*die Kontrollgruppe 2013 hat die gleiche Verteilung 
Die Altersverteilung für das Sturzjahr 2014 war etwas inhomogener mit dem höchsten Durchschnittsalter für weibliche Patienten von 86,5 Jahren in der Urologie und dem niedrigsten Durchschnittsalter von 75,9 Jahren für Patientinnen in der plastischen Chirurgie. Das höchste Durchschnittsalter männlicher Patienten war mit 82,7 Jahren in der Geriatrie zu finden (s. Tabelle 12).

Tabelle 12 Alter nach Geschlecht und behandelnder Abteilung 2014

\begin{tabular}{|c|c|c|c|c|c|c|c|c|}
\hline \multirow[t]{2}{*}{ Sturzjahr 2014} & \multicolumn{8}{|c|}{ Alter } \\
\hline & & $\mathrm{N}$ & $\%$ & Mittelwert & Std & Median & Min & Max \\
\hline Geschlecht & Abteilung & & & & & & & \\
\hline \multirow[t]{6}{*}{ männlich } & Geriatrie & 85 & 17,7 & 82,7 & 6,84 & 84 & 65 & 97 \\
\hline & $\mathrm{IM}$ & 85 & 17,7 & 80,0 & 7,48 & 81 & 65 & 94 \\
\hline & $\mathrm{UCH}$ & 17 & 3,5 & 80,3 & 7,81 & 79 & 66 & 91 \\
\hline & $\mathrm{ACH}$ & 9 & 1,9 & 79,5 & 5,64 & 80 & 69 & 88 \\
\hline & UR & 30 & 6,2 & 79,3 & 5,87 & 79 & 65 & 93 \\
\hline & $\mathrm{PCH}$ & 1 & 0,2 & 75 & & 75 & 75 & 75 \\
\hline \multirow[t]{7}{*}{ weiblich } & Geriatrie & 98 & 20,4 & 83,4 & 6,77 & 84 & 65 & 97 \\
\hline & $\mathrm{IM}$ & 81 & 16,8 & 82,7 & 6,69 & 83 & 66 & 95 \\
\hline & $\mathrm{UCH}$ & 41 & 8,5 & 82,6 & 9,24 & 83 & 65 & 97 \\
\hline & $\mathrm{ACH}$ & 15 & 3,1 & 84 & 7,48 & 84 & 69 & 95 \\
\hline & UR & 10 & 2,1 & 86,5 & 7,95 & 88,5 & 73 & 97 \\
\hline & $\mathrm{PCH}$ & 8 & 1,7 & 75,9 & 4,76 & 77 & 67 & 81 \\
\hline & HNO & 1 & 0,2 & 85 & & 85 & 85 & 85 \\
\hline
\end{tabular}

\subsection{Sturzinzidenz}

Stürze auf 1000 Behandlungstage sind eine wichtige Kennzahl zur Bestimmung der Inzidenz von Stürzen. Die meisten Stürze bezogen auf 1000 Behandlungstage ereigneten sich in der Geriatrie mit 10,5 Stürzen im Jahr 2013 und 8,9 Stürzen im Jahr 2014 (s. Tabelle 13). Die wenigsten Stürze bezogen auf 1000 Behandlungstage waren in der Abteilung der Allgemeinchirurgie zu finden. Da hier - wie bei allen Daten des Medizincontrollings - keine 
Angaben zum Patientenalter gemacht wurden, kann bei dieser Statistik kein ausschließlich geriatrisches Patientenkollektiv angenommen werden.

Tabelle 13 Stürze auf 1000 Behandlungstage

\begin{tabular}{|l|r|r|}
\hline Fachabteilung & Sturz auf 1000 Beh-tage* 2013 & Sturz auf 1000 Beh-tage* 2014 \\
\hline PCH & 4,44 & 5,69 \\
\hline ACH & 1,47 & 1,50 \\
\hline UR & 2,54 & 4,05 \\
\hline UCH & 1,91 & 1,76 \\
\hline Geriatrie & 10,48 & 8,90 \\
\hline IM & 6,68 & 6,05 \\
\hline Pneu & 3,01 & 3,28 \\
\hline Mittelwert & 4,36 & 4,46 \\
\hline
\end{tabular}

$*$ Beh-tage $=$ Behandlungstage

Die Inzidenz von Stürzen als Mittelwert aller behandelnden Abteilungen war über die Jahre 2003 bis 2014 relativ konstant und lag bei ca. 4 Stürzen auf 1000 Behandlungstage (s. Abbildung 1). 


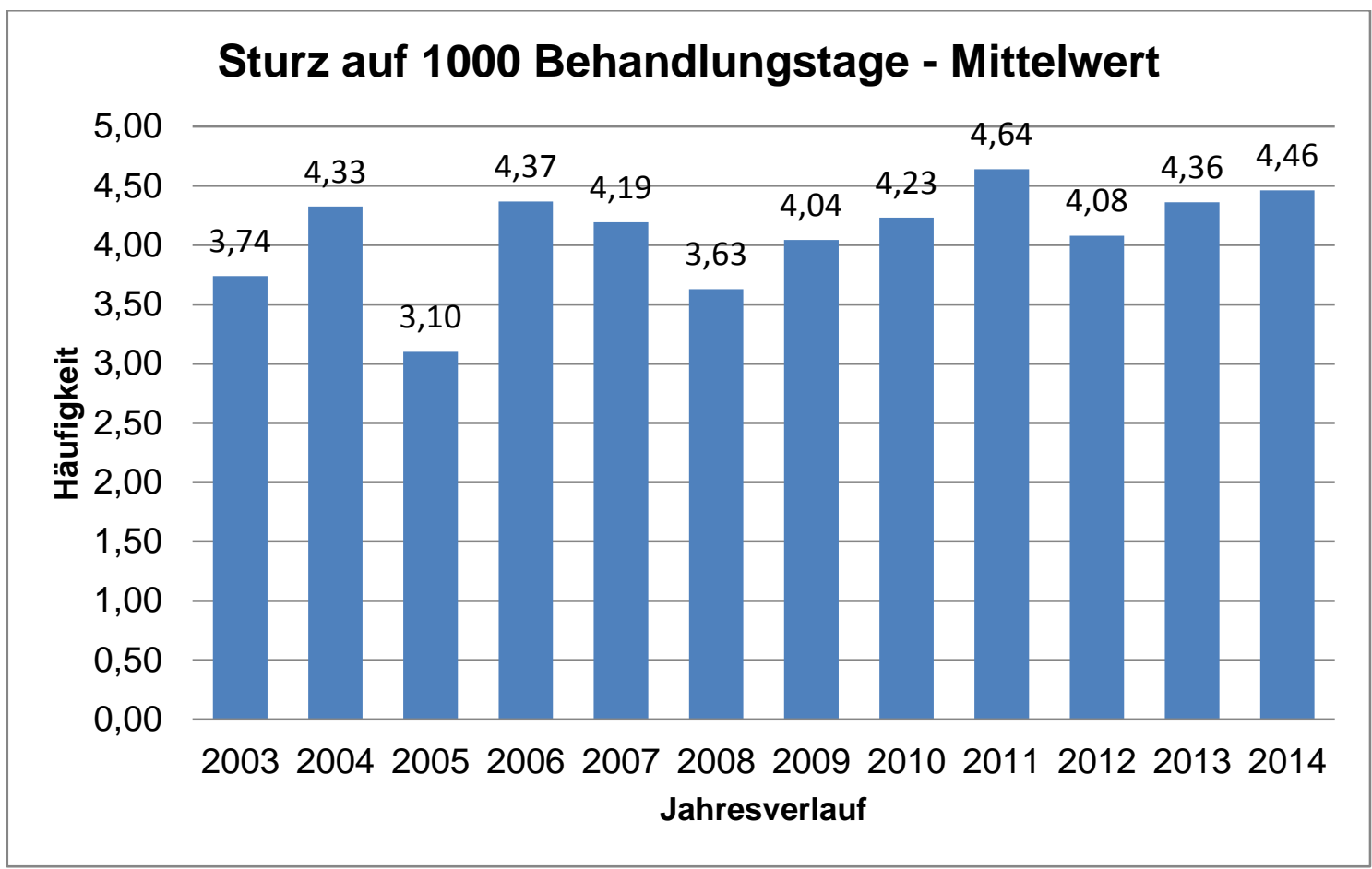

Abbildung 1 Stürze auf 1000 Behandlungstage

Die meisten Patienten (80,3\% (N=386) im Jahr 2013 und 76,3\% ( $\mathrm{N}=367)$ im Jahr 2014) stürzten lediglich einmal in ihrem Aufenthalt. Mehr als ein Sturz war somit bei 19,8\% der Gestürzten im Jahr 2013 und 23,7\% der gestürzten Patienten im Jahr 2014 zu finden. Akkumuliert ergaben sich 614 Stürze im Jahr 2013 und 637 Stürze im Jahr 2014 (s. Tabelle 14).

Tabelle 14 Sturzanzahl im Aufenthalt

\begin{tabular}{|l|r|r|r|}
\hline Sturzanzahl im Aufenthalt & Sturzjahr 2013 N (\%) & Sturzjahr 2014 N (\%) & $\begin{array}{c}\text { Summe der } \\
\text { Stürze }\end{array}$ \\
\hline 1 & $386(80,3)$ & $367(76,3)$ & 753 \\
\hline 2 & $68(14,1)$ & $79(16,4)$ & 294 \\
\hline 3 & $18(3,7)$ & $28(5,3)$ & 138 \\
\hline 4 & $7(1,5)$ & $7(1,5)$ & 56 \\
\hline 5 & $2(0,4)$ & 0 & 10 \\
\hline Summe & 481 & 481 & 1251 \\
\hline
\end{tabular}


Nachts ereigneten sich weniger Stürze als am Tag, 36,4\% (N=168) im Jahr 2013 und 40,8\% (N=192) im Jahr 2014 (s. Tabelle 15). Zu erwähnen ist, dass bei insgesamt 29 Patienten keine Angaben zum Sturzzeitpunkt gemacht wurden. Bei diesen Patienten wurde das Sturzprotokoll folglich als unvollständig gewertet (s. Tabelle 16).

Tabelle 15 Zeitpunkt des Sturzes

\begin{tabular}{|l|r|r|r|}
\hline Uhrzeit des Sturzes & Sturzjahr 2013 N (\%) & Sturzjahr 2014 N (\%) & \multicolumn{1}{c|}{ Summe } \\
\hline 06:00- 22:00 & $294(63,6)$ & $279(59,2)$ & $573(61,4)$ \\
\hline 22:00-06:00 & $168(36,4)$ & $192(40,8)$ & $360(38,6)$ \\
\hline Summe & 462 & 471 & 933 \\
\hline
\end{tabular}

29 fehlende Angaben zum Sturzzeitpunkt

\subsection{Sturzprotokolle}

Sturzprotokolle wurden in der Regel sehr sorgfältig ausgefüllt. Als unvollständig galt ein Sturzprotokoll, wenn eins der Hauptfelder nicht ausgefüllt wurde. Lediglich 11,4\% $(\mathrm{N}=110)$ bezogen auf die Gesamtzahl untersuchter Protokolle wurden unvollständig ausgefüllt (s. Tabelle 16). Ein Beispielprotokoll findet sich im Anhang.

Tabelle 16 Vollständigkeit der Sturzprotokolle

\begin{tabular}{|l|r|r|c|}
\hline Sturzprotokoll vollständig & Sturzjahr $2013 \mathrm{~N}(\%)$ & Sturzjahr $2014 \mathrm{~N}(\%)$ & \multicolumn{1}{c|}{ Summe } \\
\hline $\mathrm{Ja}$ & $417(86,7)$ & $435(90,4)$ & $852(88,6)$ \\
\hline Nein & $64(13,3)$ & $46(9,6)$ & $110(11,4)$ \\
\hline Summe & 481 & 481 & 962 \\
\hline
\end{tabular}

Um festzustellen, ob ein Unterschied zwischen Tag- und Nachtschicht im Ausfüllen der Sturzprotokolle besteht, wurde die Uhrzeit des Sturzes mit der Vollständigkeit der Sturzprotokolle in Zusammenhang gesetzt (s. Tabelle 17). Ein signifikanter Unterschied bestand allerdings nicht ( $\mathrm{p}=0,24$ errechnet mit dem Chi-Quadrat-Test). 
Tabelle 17 Zeitpunkt des Sturzes und Vollständigkeit der Sturzprotokolle

\begin{tabular}{|l|r|r|r|}
\hline \multirow{2}{*}{ Uhrzeit des Sturzes } & \multicolumn{2}{|c|}{ Sturzprotokoll vollständig N (\%) } & \multirow{2}{*}{ Summe } \\
\cline { 2 - 3 } & \multicolumn{1}{|c|}{ Ja } & \multicolumn{1}{c|}{ Nein } \\
\hline 06:00- 22:00 & $517(90,2)$ & $56(9,8)$ & $573(61,4)$ \\
\hline 22:00-06:00 & $330(92,5)$ & $27(7,5)$ & $360(38,6)$ \\
\hline Summe & $850(91,1)$ & $83(8,9)$ & 933 \\
\hline
\end{tabular}

In Abbildung 2 sind Sturzursachen des Jahres 2014 nach Häufigkeit in den jeweiligen behandelnden Abteilungen des EKW dargestellt. Gangunsicherheit $(\mathrm{N}=311$, lila) und Kraftlosigkeit $(\mathrm{N}=344$, blau) machten dabei den größten Anteil aus, wobei auch simultane Nennungen möglich waren.

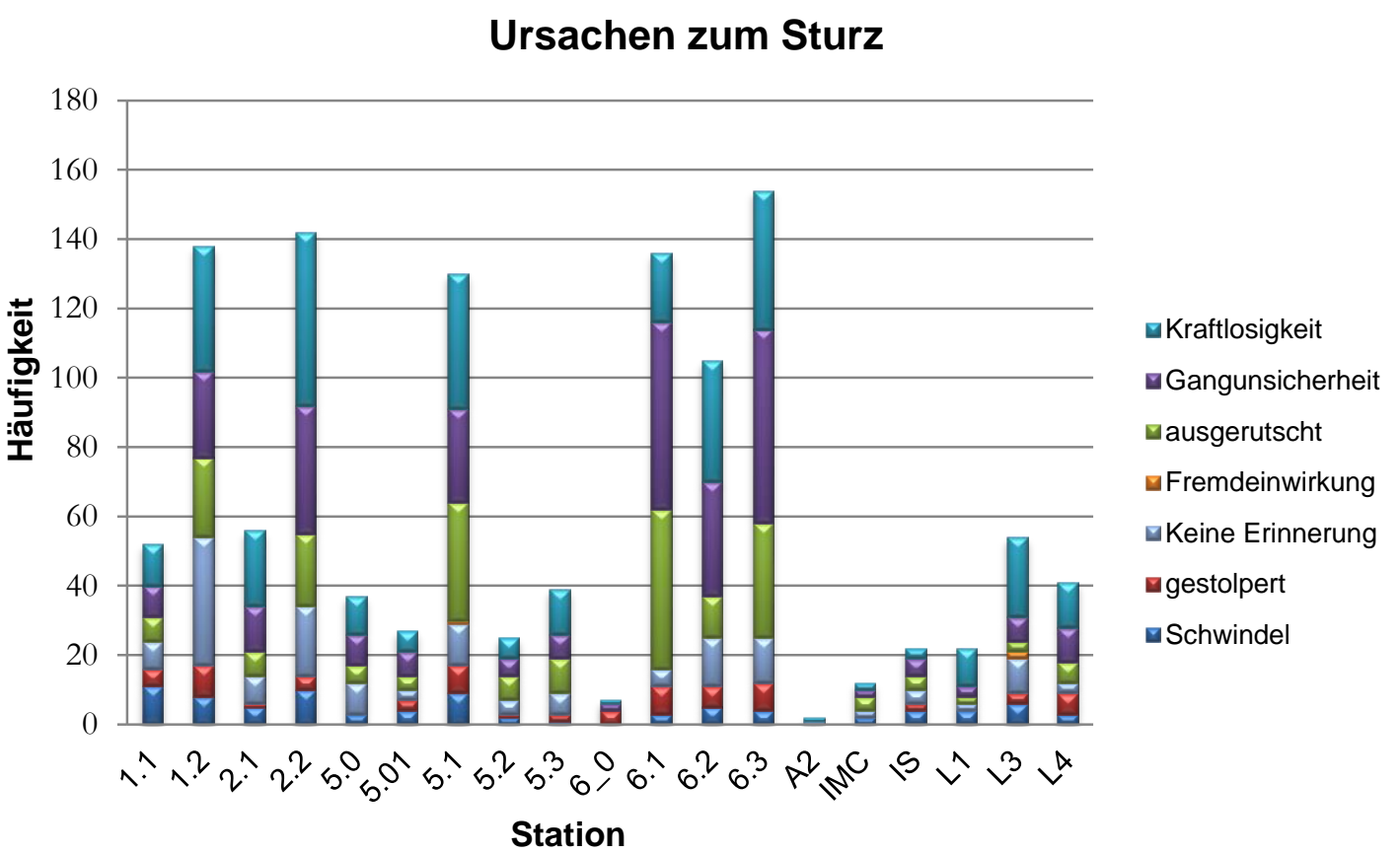

Abbildung 2 Sturzursachen nach behandelnder Abteilung 2014

\subsection{Sturzverletzungen}

Leichte- bis mittelschwere Verletzungen, wie Prellmarken oder Platzwunden, waren bei ca. 1/3 aller gestürzten Patienten zu finden und damit relativ häufig. Schwere Sturzverletzungen, also Frakturen, waren zum Glück selten. Nur 3,7\% (N=36) aller gestürzten Patienten verletzten sich schwer (s. Tabelle 18). Operations-bedürftig waren lediglich 17 der 36 schweren Sturzverletzungen. Zwei Patienten starben an den Folgen der Verletzungen. Dabei handelte es sich um eine Kalottenfraktur mit subarachnoidaler- und subduraler Blutung 
unter gerinnungshemmender Therapie sowie um eine mediale Schenkelhalsfraktur mit myokardialem Pumpversagen in der operativen Nachsorge.

Tabelle 18 Sturzverletzungen

\begin{tabular}{|c|c|c|c|}
\hline Sturzfolgen & $\begin{array}{l}\text { Sturzjahr } 2013 \quad N \\
(\%)\end{array}$ & $\begin{array}{l}\text { Sturzjahr } 2014 \quad N \\
(\%)\end{array}$ & Summe der Stürze \\
\hline $\begin{array}{l}\text { Leichte- bis mittelschwere Sturzverlet- } \\
\text { zung }\end{array}$ & $154(32,0)$ & $142(29,5)$ & $296(30,8)$ \\
\hline Schwere Sturzverletzung & $19(4,0)$ & $17(3,5)$ & $36(3,7)$ \\
\hline Schwere Sturzverletzung mit $O P^{*}$ & $6(1,3)$ & $11(2,3)$ & $17(1,8)$ \\
\hline Summe & $173(36,0)$ & $159(33,1)$ & $332(34,5)$ \\
\hline
\end{tabular}

*Zeile nicht in Gesamtsumme der Sturzverletzungen miteinberechnet

\subsection{Einflussfaktoren für Stürze}

In diesem Abschnitt der Ergebnisdokumentation werden zunächst Mittelwertunterschiede des gepaarten-t-Tests dargestellt. Diese konnten wie bereits in 3.6.2 erwähnt nur für kontinuierliche Variablen errechnet werden. Die Ergebnisse der multiplen logistischen Regression werden daran anschließend erläutert. Die Darstellung der Ergebnisse erfolgte zur Verbesserung der Übersichtlichkeit in Form mehrerer Tabellen, obwohl sich die Werte aus einem einzigen Regressionsmodell entnehmen ließen und gegenseitig bedingten. Außerdem muss erwähnt werden, dass nur die Variablen, die tatsächlich nach Vortest in das finale Regressionsmodell aufgenommen wurden, betrachtet wurden. Alle anderen Einflussvariablen sind in Tabelle 35 im Anhang zusammengefasst. Auch die relativen und absoluten Häufigkeiten der Einflussvariablen des Sturzjahres 2014, für das keine Kontrollgruppe ausgewertet wurde, sind in Tabelle 37 und Tabelle 38 im Anhang dokumentiert. Da nur in der geriatrischen Abteilung geriatrische Assessments erhoben wurden, konnten diese nicht in das Regressionsmodell eingefügt werden, weshalb lediglich nicht adjustierte Odds-Ratios errechnet wurden.

\subsubsection{Mittelwertunterschiede}

Da nach den Merkmalen Alter, Geschlecht und Abteilungszugehörigkeit zugeordnet wurde, besteht kein Unterschied im durchschnittlichen Alter für die Patienten des Sturzjahres 2013 und die Kontrollpatienten des Jahres 2013 (M=82,1, p=1). Irrtumswahrscheinlichkei- 
ten kleiner als $5 \%(\mathrm{P}<0,05)$ wurden als statistisch signifikante Differenzen gewertet. Beide Gruppen unterschieden sich signifikant im Barthel-Index (s. Tabelle 19): Während der Index bei der Sturzgruppe 38,8 (Standardabweichung $(\mathrm{SD})=19,3$ ) betrug, lag er bei der Kontrollgruppe bei 43,8 (SD 23,5). Die Differenz betrug 5,0 (95\%-KI: 0,9 - 9,21; $\mathrm{p}=$ 0,02). Ähnliches ergab sich beim Tinetti-Test. Auch hier konnte ein signifikanter Unterschied gefunden werden (95\%-KI: 0,53-3,56; $\mathrm{p}=0,01)$, da aber beide Mittelwerte (8,8 (SD 7,72) für die Kontrollgruppe und 6,5 (SD 2,02) für die Sturzgruppe) weit unter 20 Punkten lagen, bestand für beide Gruppen nach dem Tinetti-Test ein deutlich erhöhtes Sturzrisiko. Da Mittelwertunterschiede für nichtparametrische Daten nur eine eingeschränkte Gültigkeit besitzen, wurden die geriatrischen Assessments mit dem Wilcoxon-Rangsummen-Test auf statistische signifikante Verteilungsunterschiede geprüft. Dabei bestätigten sich die bereits bei den Mittelwertunterschieden errechneten Ergebnisse (Barthel-Index: $p=0,03$; TinettiTest: $\mathrm{p}=0,05)$. Es zeigte sich darüber hinaus ein statistisch signifikanter Unterschied für den Timed-Up-and-Go-Test $\mathrm{p}=0,04$.

Ebenfalls signifikant waren: gemessene Körpertemperatur am Tag des Sturzes sowie Hämoglobin- und Natriumkonzentration im Aufnahmelabor. Auch hier handelte es sich zwar um signifikante Unterschiede, die sich jedoch im klinischen Normbereich befanden. Im Abschnitt 4.5.2 erfolgte daher eine Einteilung unter klinischen Gesichtspunkten, um unter anderem genauere Angaben zum Einfluss dieser Variablen auf das Sturzrisiko zu machen. Anders verhielt es sich bei den Laborwerten CRP und Leukozyten Konzentration: Es ergaben sich gemittelte CRP- Werte von 3,8 mg/l (SD 5,62) in der Kontrollgruppe, gegenüber 5,09 mg/1 (SD 6,83) in der Sturzgruppe. Die Differenz betrug 1,3 (95\%-KI: 0,5-2,09; $\mathrm{p}=0,001)$ und wäre somit sowohl nach statistischen als auch unter klinischen Gesichtspunkten signifikant, da die Grenze für einen Entzündungsprozess bei einem CRP-Wert $>5$ $\mathrm{mg} / 1$ und einer Leukozyten Konzentration $>10.000 / \mu$ l gezogen wird.

Tabelle 19 Mittelwertunterschiede Sturzjahr 2013 und Kontrollen 2013

\begin{tabular}{|c|c|c|c|c|c|c|c|c|c|}
\hline \multirow[t]{2}{*}{ Einflussvariablen } & \multicolumn{3}{|c|}{ Kontrollen 2013} & \multicolumn{6}{|c|}{ Sturzjahr 2013} \\
\hline & $\mathrm{N}$ & MW & SD & $\mathrm{N}$ & M & SD & DIFF & $(95 \mathrm{KI})$ & $p$ \\
\hline Alter & 481 & 82,1 & 7,01 & 481 & 82,1 & 7,01 & 0 & 0 & 1 \\
\hline BMI & 285 & 26,8 & 5,45 & 274 & 25,9 & 5,43 & 0,9 & $\begin{array}{r}(-0,6- \\
1,4)\end{array}$ & 0,42 \\
\hline MMST & 71 & 22,4 & 5,76 & 117 & 21,1 & 5,82 & 1,3 & $\begin{array}{c}(-3,2- \\
1,64)\end{array}$ & 0,5 \\
\hline Barthel-Index & 209 & 43,8 & 23,51 & 205 & 38,8 & 19,32 & 5,0 & $\begin{array}{r}(1,3- \\
9,5)\end{array}$ & 0,01 \\
\hline Tinetti-Test & 183 & 8,8 & 7,72 & 156 & 6,7 & 6,46 & 2,1 & $\begin{array}{r}(0,02- \\
3,29)\end{array}$ & 0,05 \\
\hline Timed-Up-and-Go & 83 & 25,4 & 14,32 & 50 & 30 & 13 & 4,5 & $(-11,3-$ & 0,73 \\
\hline
\end{tabular}




\begin{tabular}{|c|c|c|c|c|c|c|c|c|c|}
\hline & & & & & & & & $15,8)$ & \\
\hline BD-Systolisch & 450 & 128,6 & 21,84 & 444 & 131,2 & 21,83 & 2,6 & $\begin{array}{l}(0,3- \\
5,3)\end{array}$ & 0,08 \\
\hline BD-Diastolisch & 450 & 71,8 & 10,29 & 444 & 72,7 & 11,57 & 0,9 & $\begin{array}{r}(-0,3- \\
2,7)\end{array}$ & 0,12 \\
\hline Herzfrequenz & 433 & 75,7 & 12,03 & 423 & 76,3 & 13,26 & 0,6 & $\begin{array}{r}(-0,72- \\
2,9)\end{array}$ & 0,22 \\
\hline Temperatur & 355 & 36,5 & 0,48 & 393 & 36,6 & 0,53 & 0,1 & $\begin{array}{r}(0,05- \\
0,2)\end{array}$ & 0,001 \\
\hline Blutzucker & 56 & 152,6 & 58,06 & 115 & 160,5 & 77,8 & 7,9 & $\begin{array}{r}(-47,2- \\
71,2)\end{array}$ & 0,67 \\
\hline Hämoglobin & 481 & 11,8 & 2,09 & 481 & 11,4 & 2,05 & 0,4 & $\begin{array}{r}(0,1- \\
0,6)\end{array}$ & 0,01 \\
\hline Kreatinin & 481 & 1,2 & 0,78 & 480 & 1,3 & 0,84 & 0,1 & $\begin{array}{r}(-0,04- \\
0,1) \\
\end{array}$ & 0,25 \\
\hline Natrium & 481 & 136,9 & 5,1 & 480 & 136,1 & 5,38 & 0,8 & $\begin{array}{r}(0,1- \\
1,5)\end{array}$ & 0,02 \\
\hline Kalium & 481 & 4,1 & 0,6 & 480 & 4,1 & 0,62 & 0,0 & $\begin{array}{l}(-0,1- \\
0,05)\end{array}$ & 0,43 \\
\hline Kalzium & 469 & 2,3 & 0,19 & 475 & 2,3 & 0,18 & 0,0 & $\begin{array}{r}(-0,03- \\
0,02)\end{array}$ & 0,26 \\
\hline CRP & 476 & 3,8 & 5,62 & 476 & 5,1 & 6,83 & 1,3 & $\begin{array}{r}(0,4- \\
2,1) \\
\end{array}$ & 0,002 \\
\hline Leukozyten & 481 & 9,4 & 4,35 & 480 & 10,2 & 5,71 & 0,8 & $\begin{array}{r}(0,1- \\
1,5)\end{array}$ & 0,02 \\
\hline
\end{tabular}

$\mathrm{MW}=$ Mittelwert, $\mathrm{SD}=$ Standardabweichung, $\mathrm{DIFF}=$ Differenz, $(95-\mathrm{KI})=95 \%-$ Konfidenzintervall, $\mathrm{p}=\mathrm{p}-\mathrm{Wert}$

Auf die Mittelwertunterschiede zwischen dem Sturzjahr 2013 und dem Sturzjahr 2014 soll nur kurz eingegangen werden, da sich die Gruppen anders zusammensetzten und somit nur bedingt vergleichbar waren. Zu erwähnen ist, dass sich die Gruppen im Altersdurschnitt stark ähnelten. Die Differenz betrug 0,11 bei $M=82,1$ im Sturzjahr 2013 und M=82,0 im Sturzjahr 2014. Gleiches galt für den MMST mit M=21,1 im Sturzjahr 2013 und M=21,3 im Sturzjahr 2014, allerdings, wie bereits erwähnt, nur mit Erfassungen in der geriatrischen Abteilung. Außerdem wiesen die behandelten Patienten im Sturzjahr 2014 im Gegensatz zu den Patienten im Sturzjahr 2013 im Aufnahmelabor in der gemittelten CRP und der Leukozyten Konzentration keine Zeichen für Entzündungsprozesse auf (s. Tabelle 20).

Tabelle 20 Mittelwertunterschiede Sturzjahr 2013 und Sturzjahr 2014

\begin{tabular}{|l|c|c|c|c|c|c|c|c|c|}
\hline \multirow{2}{*}{ Einflussvariablen } & \multicolumn{3}{|c|}{ Sturzjahr 2014} & \multicolumn{7}{|c|}{ Sturzjahr 2013} \\
\cline { 2 - 11 } & N & MW & \multicolumn{1}{c|}{ SD } & N & M & \multicolumn{1}{c|}{ SD } & DIFF & $(95 \mathrm{KI})$ & \multicolumn{1}{c|}{ p } \\
\hline Alter & 481 & 82,0 & 7,27 & 481 & 82,1 & 7,01 & 0,1 & $(-0,80-1,01)$ & 0,81 \\
\hline BMI & 264 & 25,7 & 5,41 & 274 & 25,9 & 5,43 & 0,2 & $(-0,65-1,19)$ & 0,57 \\
\hline
\end{tabular}




\begin{tabular}{|l|r|r|r|r|r|r|r|r|r|}
\hline MMST & 82 & 21,3 & 6,06 & 117 & 21,1 & 5,82 & 0,2 & $(-1,53-1,86)$ & 0,84 \\
\hline Barthel-Index & 181 & 41,5 & 18,11 & 205 & 38,8 & 19,32 & 2,7 & $(-1,02-6,47)$ & 0,15 \\
\hline Tinetti-Test & 139 & 8,5 & 6,64 & 156 & 6,7 & 6,46 & 1,7 & $(0,24-3,25)$ & 0,02 \\
\hline Timed-Up-and-Go & 43 & 34,0 & 16,2 & 50 & 30 & 13 & 4,0 & $(-2,11-10,15)$ & 0,19 \\
\hline BD-Systolisch & 459 & 127,8 & 21,9 & 444 & 131,2 & 21,83 & 3,4 & $(0,52-6,24)$ & 0,02 \\
\hline BD-Diastolisch & 459 & 71,7 & 10,91 & 444 & 72,7 & 11,57 & 1,0 & $(-0,48-2,45)$ & 0,19 \\
\hline Herzfrequenz & 439 & 77,3 & 12,43 & 423 & 76,3 & 13,26 & 1,0 & $(-0,68-2,76)$ & 0,24 \\
\hline Temperatur & 406 & 36,6 & 0,48 & 393 & 36,6 & 0,53 & 0,0 & $(-0,04-0,12)$ & 0,33 \\
\hline Blutzucker & 91 & 157,0 & 57,61 & 115 & 160,5 & 77,8 & 3,5 & $(-15,1-22,16)$ & 0,7 \\
\hline Hämoglobin & 481 & 11,5 & 2,03 & 481 & 11,4 & 2,05 & 0,1 & $(-0,15-0,36)$ & 0,41 \\
\hline Kreatinin & 481 & 1,4 & 1,07 & 480 & 1,3 & 0,84 & 0,1 & $(-0,01-0,23)$ & 0,06 \\
\hline Natrium & 481 & 136,8 & 5,36 & 480 & 136,1 & 5,38 & 0,7 & $(0,05-1,4)$ & 0,04 \\
\hline Kalium & 481 & 4,1 & 0,62 & 480 & 4,1 & 0,62 & 0,0 & $(-0,03-0,13)$ & 0,2 \\
\hline Kalzium & 455 & 2,2 & 0,19 & 475 & 2,3 & 0,18 & 0,1 & $(0,002-0,05)$ & 0,03 \\
\hline CRP & 466 & 4,8 & 7,04 & 476 & 5,1 & 6,83 & 0,3 & $(-0,63-1,14)$ & 0,57 \\
\hline Leukozyten & 481 & 9,4 & 4,01 & 480 & 10,2 & 5,71 & 0,8 & $(0,11-1,36)$ & 0,02 \\
\hline
\end{tabular}

\subsubsection{Einflussfaktoren auf das Sturzrisiko}

Einen signifikanten Einfluss unter den psychoaktiven Medikamenten auf das Sturzrisiko zeigten: lang wirksame Benzodiazepine, SNRI, Z-Substanzen und sedierende Neuroleptika. Das Risiko, im stationären Aufenthalt zu stürzen, war unter Einnahme von lang wirksamen Benzodiazepinen mehr als dreimal (OR 3,49) so hoch (s. Tabelle 21). Bromazepam (N=4) und Nitrazepam ( $\mathrm{N}=3$ ) wurden dabei am häufigsten verordnet und ausschließlich in der Sturzgruppe eingenommen (s. Tabelle 33). Die Einnahme von SNRI erhöhte das Risiko für einen Sturz um mehr als das doppelte (OR 2,51), bei deutlich höheren Verordnungszahlen $5,6 \%(\mathrm{~N}=27)$ in der Sturzgruppe und 2,9\% $(\mathrm{N}=14)$ in der Kontrollgruppe. Bei den weiteren psychoaktiven Medikamenten zeigte sich nur im univariaten Modell ein moderater Einfluss.

Tabelle 21 Einfluss psychoaktiver Medikamente auf das Sturzrisiko

\begin{tabular}{|l|r|r|r|r|r|r|}
\hline $\begin{array}{c}\text { Psychoaktive Medi- } \\
\text { kation }\end{array}$ & N & $\%$ & univariate OR (95 KI) & $p$ & adjustierte OR (95 KI) & $p$ \\
\hline Benzodiazepine kurz- und mittellang wirksam & \\
\hline Sturz & 68 & 14,1 & $1,36(0,92-2,0)$ & 0,12 & & \\
\hline Kein Sturz & 52 & 10,8 & & & & \\
\hline
\end{tabular}




\begin{tabular}{|c|c|c|c|c|c|c|}
\hline Sturz & 14 & 1,9 & $2,85(1,02-7,99)$ & 0,04 & $3,49(1,16-10,523)$ & 0,03 \\
\hline Kein Sturz & 5 & 1,0 & & & & \\
\hline \multicolumn{7}{|c|}{ Neuroleptika sedierend } \\
\hline Sturz & 44 & 9,2 & $1,76(1,07-2,91)$ & 0,03 & $1,87(1,08-3,23)$ & 0,03 \\
\hline Kein Sturz & 26 & 5,4 & & & & \\
\hline \multicolumn{7}{|l|}{ SNRI } \\
\hline Sturz & 27 & 5,6 & $1,98(1,03-3,83)$ & 0,04 & $2,51(1,23-5,12)$ & 0,02 \\
\hline Kein Sturz & 14 & 2,9 & & & & \\
\hline \multicolumn{7}{|l|}{ SSRI } \\
\hline Sturz & 50 & 10,4 & $1,39(0,89-2,17)$ & 0,14 & & \\
\hline Kein Sturz & 37 & 7,7 & & & & \\
\hline \multicolumn{7}{|c|}{ Tetrazyklische Antidepressiva } \\
\hline Sturz & 83 & 17,2 & $1,44(1,01-2,05)$ & 0,05 & & \\
\hline Kein Sturz & 61 & 12,7 & & & & \\
\hline \multicolumn{7}{|l|}{ Z-Substanz } \\
\hline Sturz & 55 & 11,4 & $1,75(1,12-2,75)$ & 0,01 & $2,29(1,38-3,59)$ & 0,001 \\
\hline Kein Sturz & 33 & 6,9 & & & & \\
\hline
\end{tabular}

Unter den nicht psychoaktiven Medikamenten erhöhten lediglich ACE-Hemmer und Sartane (OR 1,42) leicht das Sturzrisiko (s. Tabelle 22). Einen signifikanten jedoch das Sturzrisiko reduzierenden Einfluss hatten Aldosteronrezeptor-Antagonisten (OR 0,54), unter denen fast ausschließlich Spironolacton N=31 in der Sturzgruppe und N=50 in Kontrollgruppe eingenommen wurde. Antiarrhythmika, die nach Kriterien der PRISCUS-Liste nicht empfohlen werden, hier nur das Medikament Digoxin, hatten erstaunlicherweise ebenfalls einen das Sturzrisiko reduzierenden signifikanten Einfluss (OR 0,32). Antiarrhythmika, die nach den Kriterien der PRISCUS-Liste empfohlen wurden, hatten insgesamt keinen Einfluss auf das Sturzrisiko $(p=0,39)$. Allerdings wurde Amiodaron in der Sturzgruppe $\mathrm{N}=20$ wesentlich häufiger verschrieben als in der Kontrollgruppe $\mathrm{N}=5$ (s. Tabelle 33). Opiate zeigten nur in der univariaten Analyse einen leichten statistisch signifikanten Einfluss und wurden im multivariaten Regressionsverfahren aus dem Modell entfernt.

Tabelle 22 Einfluss nicht psychoaktiver Medikamente auf das Sturzrisiko

\begin{tabular}{|c|c|c|c|c|c|c|}
\hline $\begin{array}{l}\text { Nicht Psychoaktive } \\
\text { Medikation }\end{array}$ & $\mathbf{N}$ & $\%$ & univariate OR (95KI) & $\mathbf{p}$ & adjustierte OR (95KI) & $\mathbf{p}$ \\
\hline \multicolumn{7}{|c|}{ ACE-Hemmer/Sartane } \\
\hline Sturz & 308 & 64,0 & $1,33(1,03-1,73)$ & 0,03 & $1,42(1,07-1,89)$ & 0,03 \\
\hline Kein Sturz & 275 & 57,2 & & & & \\
\hline
\end{tabular}




\begin{tabular}{|c|c|c|c|c|c|c|}
\hline Sturz & 33 & 6,9 & $0,58(0,37-0,92)$ & 0,02 & $0,54(0,33-0,88)$ & 0,03 \\
\hline Kein Sturz & 54 & 11,2 & & & & \\
\hline \multicolumn{7}{|c|}{ Antihypertensiva nach PRISCUS-Liste nicht empfohlen } \\
\hline Sturz & 3 & 0,6 & $0,42(0,11-1,65)$ & 0,2 & & \\
\hline Kein Sturz & 7 & 1,5 & & & & \\
\hline \multicolumn{7}{|c|}{ Antiarrhythmika nach PRISCUS-Liste nicht empfohlen } \\
\hline Sturz & 4 & 0,4 & $0,22(0,08-0,68)$ & 0,004 & $0,32(0,11-0,99)$ & 0,04 \\
\hline Kein Sturz & 17 & 3,5 & & & & \\
\hline \multicolumn{7}{|l|}{ Opiate } \\
\hline Sturz & 151 & 31,4 & $0,85(0,65-1,12)$ & 0,24 & & \\
\hline Kein Sturz & 168 & 34,9 & & & & \\
\hline
\end{tabular}

Unter den Laborwerten zeigten lediglich die Hyponatriämie (OR 1,52) und die Leukozytose (OR 1,39) einen schwachen signifikanten Einfluss (s. Tabelle 23). Die Leukozytose als Zeichen einer Entzündung war bereits in 4.5 .1 als ein Risikofaktor für Stürze identifiziert worden und bestätigte sich nun auch in der multivariaten Analyse.

Tabelle 23 Einfluss verschiedener Laborwerte auf das Sturzrisiko

\begin{tabular}{|c|c|c|c|c|c|c|}
\hline Laborwerte* & $\mathbf{N}$ & $\%$ & univariate OR (95KI) & $\mathbf{p}$ & adjustierte OR (95KI) & $\mathbf{p}$ \\
\hline \multicolumn{7}{|l|}{ Anämie } \\
\hline Sturz & 329 & 68,4 & $1,33(1,02-1,73)$ & 0,04 & & \\
\hline kein Sturz & 298 & 62,0 & & & & \\
\hline \multicolumn{7}{|l|}{ Hyponatriämie } \\
\hline Sturz & 200 & 41,6 & $1,49(1,15-1,95)$ & 0,003 & $1,52(1,15-2,03)$ & 0,003 \\
\hline Kein Sturz & 155 & 32,2 & & & & \\
\hline \multicolumn{7}{|l|}{ Leukozytopenie } \\
\hline Sturz & 18 & 3,7 & $1,83(0,84-4,01)$ & 0,12 & & \\
\hline Kein Sturz & 10 & 2,1 & & & & \\
\hline \multicolumn{7}{|l|}{ Leukozytose } \\
\hline Sturz & 188 & 39,1 & $1,40(1,08-1,83)$ & 0,01 & $1,39(1,05-1,87)$ & 0,02 \\
\hline Kein Sturz & 151 & 31,4 & & & & \\
\hline \multicolumn{7}{|l|}{$C R P>5 m g / l$} \\
\hline Sturz & 153 & 31,8 & $1,29(0,91-1,84)$ & 0,02 & & \\
\hline Kein Sturz & 120 & 25,0 & & & & \\
\hline
\end{tabular}

*im Aufnahmenlabor

Die Nebendiagnose Delir wies ein fast vierfach erhöhtes Risiko (OR 3,74), im Aufenthalt zu stürzen auf und war somit die stärkste das Sturzrisiko erhöhende Variable im Modell (s. 
Tabelle 24). Ebenfalls einen deutlichen Einfluss zeigten die Nebendiagnosen Exsikkose und Parkinsonsyndrom. Bei der Einteilung galten die in 4.5.3 beschriebenen Kriterien.

Tabelle 24 Einfluss der Nebendiagnosen auf das Sturzrisiko

\begin{tabular}{|c|c|c|c|c|c|c|}
\hline Nebendiagnosen & $\mathrm{N}$ & $\%$ & univariate OR (95KI) & $\mathrm{p}$ & adjustierte OR (95KI) & $\mathrm{p}$ \\
\hline \multicolumn{7}{|l|}{ Parkinsonsyndrom } \\
\hline Sturz & 36 & 7,5 & $2,08(1,16-3,72)$ & 0,01 & $2,38(1,27-4,46)$ & 0,02 \\
\hline Kein Sturz & 18 & 3,7 & & & & \\
\hline \multicolumn{7}{|l|}{ Demenz } \\
\hline Sturz & 95 & 19,8 & $1,29(0,93-1,80)$ & 0,13 & & \\
\hline Kein Sturz & 77 & 16,0 & & & & \\
\hline \multicolumn{7}{|c|}{ Ischämischer Schlaganfall } \\
\hline Sturz & 92 & 19,1 & $1,25(0,89-1,73)$ & 0,2 & & \\
\hline Kein Sturz & 77 & 16,0 & & & & \\
\hline \multicolumn{7}{|l|}{ Exsikkose } \\
\hline Sturz & 108 & 22,5 & $1,92(1,37-2,7)$ & 0,0001 & $1,85(1,28-2,67)$ & 0,0002 \\
\hline Kein Sturz & 63 & 13 & & & & \\
\hline \multicolumn{7}{|l|}{ Delir } \\
\hline Sturz & 81 & 16,8 & $4,03(2,49-6,53)$ & 0,0001 & $3,74(2,26-6,21)$ & 0,0001 \\
\hline Kein Sturz & 23 & 4,8 & & & & \\
\hline
\end{tabular}

Die Hauptdiagnosegruppen nach ICD-10, die signifikanten Einfluss auf das Sturzrisiko zeigten, waren Neubildungen (OR 1,85) mit einem erhöhten Risiko und Krankheiten des Verdauungssystems (OR 0,49) mit einem erniedrigten Risiko (s. Tabelle 25) zu stürzen. Jedoch gilt anzumerken, dass natürlich eine Vielzahl von Diagnosen in einer Hauptgruppe zusammengefasst wurden und somit keine Aussage über eine einzelne Hauptdiagnose getroffen werden kann.

Im Anschluss an die multiple logistische Regressionsanalyse wurde der HosmerLemeshow-Test durchgeführt. Mit einem p-Wert von 0,89 zeigte sich eine sehr gute Modellanpassung an die vorliegenden Daten.

Tabelle 25 Einfluss der Hauptdiagnose auf das Sturzrisiko 


\begin{tabular}{|c|c|c|c|c|c|c|}
\hline Sturz & 53 & 11,0 & $1,68(1,07-2,65)$ & 0,02 & $1,85(1,13-3,01)$ & 0,03 \\
\hline Kein Sturz & 33 & 6,9 & & & & \\
\hline \multicolumn{7}{|c|}{ K00-K93 Krankheiten des Verdauungssystems } \\
\hline Sturz & 32 & 6,6 & $0,54(0,34-0,85)$ & 0,01 & $0,49(0,29-0,81)$ & 0,003 \\
\hline Kein Sturz & 56 & 11,6 & & & & \\
\hline \multicolumn{7}{|c|}{ M00-M99 Krankheiten des Muskel-Skelett-Systems und des Bindegewebes } \\
\hline Sturz & 38 & 7,9 & $0,69(0,45-1,07)$ & 0,1 & & \\
\hline Kein Sturz & 53 & 11,0 & & & & \\
\hline \multicolumn{7}{|c|}{ N00-N99 Krankheiten des Urogenitalsystems } \\
\hline Sturz & 50 & 10,4 & $1,48(0,94-2,32)$ & 0,09 & & \\
\hline Kein Sturz & 35 & 7,3 & & & & \\
\hline \multicolumn{7}{|c|}{ S00-T98 Verletzungen, Vergiftungen und bestimmte andere Folgen äußerer Ursachen } \\
\hline Sturz & 97 & 20,2 & $1,25(0,90-1,73)$ & 0,18 & & \\
\hline Kein Sturz & 81 & 16,8 & & & & \\
\hline
\end{tabular}

Für den BMI, die Vitalparameter am Sturztag und die geriatrischen Assessments war es aufgrund zu weniger Beobachtungen nicht möglich, adjustierte Odds-Ratios zu berechnen, da sonst die Modellgüte stark reduziert worden wäre. Daher beschränken sich die folgenden Angaben nur auf univariate Odds-Ratios und sind nicht für den Einfluss anderer Variablen kontrolliert. Der BMI wurde lediglich bei $\mathrm{N}=274$ Patienten in der Sturzgruppe und $\mathrm{N}=285$ Patienten in der Kontrollgruppe berechnet. Ein signifikanter Zusammenhang mit einem erhöhten Sturzrisiko bestand für einen BMI $<17,5$ (s. Tabelle 26). Untergewichtige Patienten schienen somit gefährdeter für Stürze zu sein als norm- oder übergewichtige Patienten.

Tabelle 26 Einfluss des BMI auf das Sturzrisiko

\begin{tabular}{|c|c|c|c|c|}
\hline BMI & $\mathbf{N}$ & $\%$ & univariate OR (95KI) & $\mathbf{p}$ \\
\hline \multicolumn{5}{|l|}{ BMI 25-30 } \\
\hline Sturz & 98 & 35,8 & $1,03(0,72-1,46)$ & 0,87 \\
\hline Kein Sturz & 100 & 35,1 & & \\
\hline \multicolumn{5}{|l|}{$B M I>30$} \\
\hline Sturz & 48 & 17,5 & $0,70(0,47-1,07)$ & 0,1 \\
\hline
\end{tabular}




\begin{tabular}{|l|r|r|r|r|}
\hline Kein Sturz & 66 & 23,2 & & \\
\hline$B M I<17,5$ & 20 & 7,3 & $2,72(1,18-6,31)$ & 0,02 \\
\hline Sturz & 8 & 2,8 & & \\
\hline Kein Sturz & & & \\
\hline
\end{tabular}

Der BMI wurde in 559 der Fälle erhoben (N=274) im Sturzjahr 2013, (N=285) bei den Kontrollen 2013

Ein erhöhter Blutdruck von systolisch über $140 \mathrm{mmHg}$ und diastolisch über $90 \mathrm{mmHg}$, zeigte sich in der univariaten Analyse als ein Risikofaktor für Stürze $(p=0,02)$ (s. Tabelle 27).

Tabelle 27 Einfluss der Vitalparameter auf das Sturzrisiko

\begin{tabular}{|c|c|c|c|c|}
\hline $\begin{array}{l}\text { Vitalparameter am Sturztag/ Tag } \\
\text { 5. nach Aufnahme }\end{array}$ & $\mathbf{N}$ & $\%$ & univariate OR (95KI) & $\mathbf{p}$ \\
\hline \multicolumn{5}{|l|}{ Blutdruck > 140/90 } \\
\hline Sturz & 14 & 3,2 & $3,63(1,18-11,15)$ & 0,02 \\
\hline Kein Sturz & 4 & 0,9 & & \\
\hline \multicolumn{5}{|l|}{ Blutdruck <90/70 } \\
\hline Sturz & 2 & 0,4 & $0,5(0,04-5,6)$ & 1 \\
\hline Kein Sturz & 1 & 0,2 & & \\
\hline \multicolumn{5}{|l|}{ Temperatur $>37,5$} \\
\hline Sturz & 12 & 3,1 & $1,09(0,46-2,54)$ & 0,84 \\
\hline Kein Sturz & 10 & 2,8 & & \\
\hline \multicolumn{5}{|l|}{ Herzfrequenz >100 } \\
\hline Sturz & 18 & 4,3 & $1,56(0,74-3,28)$ & 0,24 \\
\hline Kein Sturz & 12 & 2,8 & & \\
\hline \multicolumn{5}{|l|}{ Herzfrequenz $<60$} \\
\hline Sturz & 18 & 4,3 & $1,23(0,62-2,49)$ & 0,54 \\
\hline Kein Sturz & 15 & 3,5 & & \\
\hline
\end{tabular}

Blutdruck Beobachtungen gesamt N=894: Stürze 2013 N=444, Kontrollen 2013 N=450; Temperatur Beobachtungen gesamt N=748: Stürze 2013 N=393, Kontrollen 2013 N=355; Herzfrequenz Beobachtungen gesamt N=856: Stürze 2013 $\mathrm{N}=423$, Kontrollen $2013 \mathrm{~N}=433$

Die Betrachtung der geriatrischen Assessments kann nur unter Berücksichtigung der jeweiligen Beobachtungszahlen erfolgen. Der Timed-Up-and-Go-Test wurde beispielsweise in der Sturzgruppe 2013 lediglich bei N=50 Patienten durchgeführt. In der Kontrollgruppe waren es immerhin N=83. Beide Gruppen wurden zu großen Teilen (Sturzjahr 2013 96\% und Kontrollen 2013 87,5\%) mit diesem Test als langsam bewertet (s. Tabelle 28). Punktwerte im Tinetti-Test von $<20$ Punkten zeigten einen signifikanten Zusammenhang $\mathrm{p}=0,05$ 
mit dem Sturzrisiko. Allerdings gilt anzumerken, dass bei den Mittelwertunterschieden zwar ein signifikanter Unterschied gezeigt werden konnte, beide Gruppen im Mittel jedoch relativ niedrige Punktwerte aufwiesen. Der MMST zeigte in keinem seiner Wertebereiche einen statistisch signifikanten Einfluss auf das Sturzrisiko.

Die Geriatrischen Assessments im Sturzjahr 2014 wurden ebenfalls ausschließlich in der geriatrischen Abteilung erhoben. Eine Zusammenfassung zum Abschneiden der Sturzgruppe 2014 in den einzelnen Tests befindet sich in Tabelle 37 im Anhang dieser Arbeit. Die Sturzjahre 2013 und 2014 zeigten in der Häufigkeitsausprägung der einzelnen Testergebnisse eine deutliche Ähnlichkeit. Die Patienten im Sturzjahr 2014 schnitten jedoch im Barthel-Index und im Tinetti-Test besser ab. Im Sturzjahr 2014 waren 29,1\% (N=53) gegenüber 43,1\% (N=89) im Sturzjahr 2013 im Barthel-Index als pflegebedürftig eingestuft worden. Im Tinetti-Test lagen 2014 91,4\% (N=127) der gestürzten Patienten unter 20 Punkten. Bereits bei den Mittelwerten war ein signifikanter Unterschied $(p=0,02)$ aufgefallen, der sich nun bestätigte. Somit kann nach den Kriterien des Barthel-Index und des Tinetti-Tests der Sturzgruppe 2014 ein etwas besserer physischer Zustand bescheinigt werden als der Sturzgruppe 2013.

Tabelle 28 Einfluss der geriatrischen Assessments auf das Sturzrisiko

\begin{tabular}{|c|c|c|c|c|}
\hline Geriatrische Assessments & $\mathbf{N}$ & $\%$ & univariate OR (95KI) & $\mathbf{p}$ \\
\hline \multicolumn{5}{|l|}{ Timed-Up-and-Go schnell } \\
\hline Sturz & 1 & 2 & $0,54(0,06-5,38)$ & 0 \\
\hline Kein Sturz & 3 & 2,6 & & \\
\hline \multicolumn{5}{|l|}{ Timed-Up-and-Go intermediate } \\
\hline Sturz & 1 & 2 & $0,83(0,07-9,36)$ & 0 \\
\hline Kein Sturz & 2 & 2,4 & & \\
\hline \multicolumn{5}{|l|}{ Timed-Up-and-Go langsam } \\
\hline Sturz & 48 & 96 & $3,28(0,69-15.66)$ & 0,12 \\
\hline Kein Sturz & 73 & 87,5 & & \\
\hline
\end{tabular}




\begin{tabular}{|c|c|c|c|c|}
\hline Sturz & 89 & 43,4 & $1,34(0,9-1,99)$ & 0,14 \\
\hline Kein Sturz & 76 & 36,4 & & \\
\hline \multicolumn{5}{|c|}{ Barthel-Index hilfsbedürftig } \\
\hline Sturz & 117 & 56,0 & $0,94(0,64-1,40)$ & 0,78 \\
\hline Kein Sturz & 112 & 54,6 & & \\
\hline \multicolumn{5}{|c|}{ Barthel-Index punktuell pflegebedürftig } \\
\hline Sturz & 12 & 5,9 & $0,5(0,24-1,04)$ & 0,06 \\
\hline Kein Sturz & 23 & 11,0 & & \\
\hline \multicolumn{5}{|c|}{ Tinetti-Test $<20$} \\
\hline Sturz & 148 & 94,9 & $2,27(0,97-5,3)$ & 0,05 \\
\hline Kein Sturz & 163 & 89,1 & & \\
\hline \multicolumn{5}{|c|}{ MMST- schwere Demenz } \\
\hline Sturz & 5 & 4,3 & $1,02(1-1,08)$ & 0,15 \\
\hline Kein Sturz & 0 & 0 & & \\
\hline \multicolumn{5}{|c|}{ MMST- moderate Demenz } \\
\hline Sturz & 47 & 40,2 & $1,03(0,6-1,9)$ & 0,9 \\
\hline Kein Sturz & 28 & 39,4 & & \\
\hline \multicolumn{5}{|c|}{ MMST- leichte Demenz } \\
\hline Sturz & 33 & 28,2 & $1,24(0,63-2,46)$ & 0,52 \\
\hline Kein Sturz & 17 & 23,9 & & \\
\hline \multicolumn{5}{|c|}{ MMST- keine Demenz } \\
\hline Sturz & 25 & 21,4 & $0,8(0,4-1,6)$ & 0,52 \\
\hline Kein Sturz & 18 & 25,4 & & \\
\hline
\end{tabular}

Timed-Up-and-Go gesamt: N=133; Sturzjahr 2013 N=50 Kontrollen 2013 N=83 ; Barthel-Index gesamt: N=414; Sturzjahr 2013 N=205 Kontrollen 2013 N=209 ; Tinetti-Test gesamt: N=339; Sturzjahr 2013 N=148 Kontrollen N=163 ; MMST gesamt: N=188; Sturzjahr 2013 N=117 Kontrollen N=71

\subsubsection{Einfluss von Menge und Dosis ausgewählter Medikamente auf das Sturzrisiko}

Zunächst sollte erfasst werden, wie viele Präparate mit psychoaktiver Wirkung ein Patient vor seinem Sturz erhalten hatte - unabhängig davon, um welches Präparat es sich genau handelte. Der Wilcoxon-Rangsummen-Test zeigte dann, dass sich die beiden Gruppen bezüglich der eingenommenen Anzahl psychoaktiver Medikamente signifikant unterschieden $(\mathrm{p}<0,001)$. Die Stärke des mit dem Kontingenz-Koeffizienten nach Pearson errechneten Zusammenhangs betrug 0,16, was eher für einen schwachen Zusammenhang spricht. Bemerkenswert war, dass 5,2\% (N=25) der Patienten in der Sturzgruppe gegenüber 1,8\% $(\mathrm{N}=9)$ in der Kontrollgruppe drei Medikamente mit psychoaktiver Wirkung eingenommen hatten (s. Tabelle 29). Ein Patient erhielt sogar fünf verschiedene Präparate gleichzeitig. 
An die beschriebene Thematik anschließend gibt Tabelle 34 im Anhang Auskunft über mögliche Doppelmedikationen von psychoaktiven Medikamenten. Die häufigste Kombination war ein SSRI mit einem tetrazyklischem Antidepressivum (fast ausschließlich Mirtazapin). Im Sturzjahr 2013 erhielten 3,1\% (N=15) der Patienten und bei den Kontrollen 2013 2,2\% ( $=11)$ Patienten eine solche Medikation. Im Sturzjahr 14 war die Kombination aus einem Benzodiazepin mit einem tetrazyklischen Antidepressivum mit 5,6\% $(\mathrm{N}=27)$ die häufigste Kombination (s. Tabelle 37). Die einzigen beiden Kombinationen, die nach der univariaten Analyse einen signifikanten Zusammenhang mit dem Sturzrisiko anzeigten, waren die Kombination eines sedierenden Neuroleptikums mit einem tetrazyklischem Antidepressivum $(\mathrm{p}=0,01)$ und ein sedierendes Neuroleptikum mit einem Benzodiazepin $(\mathrm{p}=0,03)$. Kombinationen von zwei Benzodiazepinen oder von einem Benzodiazepin und eine Z-Substanz waren sehr selten. So erhielten 0,8\% (N=4) der Patienten im Sturzjahr 2013 und 0\% (N=0) bei den Kontrollen 2013 gleichzeitig zwei Benzodiazepin-Präparate. Ein Benzodiazepin und eine Z-Substanz wurde 1,7\% $(\mathrm{N}=8)$ der Patienten im Sturzjahr 2013 und 0,4\% (N=2) der Kontrollpatienten verabreicht.

Tabelle 29 Anzahl eingenommener Psychopharmaka im Jahr 2013

\begin{tabular}{|l|r|r|r|r|r|r|r|}
\hline $\begin{array}{l}\text { Anzahl } \\
\text { psychoaktiver } \begin{array}{r}\text { Medikamente } \\
2013\end{array}\end{array}$ & \multicolumn{1}{c|}{0} & \multicolumn{1}{c|}{1} & \multicolumn{1}{c|}{3} & \multicolumn{1}{c|}{5} & Summe \\
\hline Sturz & $172(35,8)$ & $192(39,9)$ & $84(17,5)$ & $25(5,2)$ & $7(1,5)$ & $1(0,2)$ & 481 \\
\hline Kein Sturz & $233(48,4)$ & $163(33,9)$ & $69(14,4)$ & $9(1,8)$ & $7(1,5)$ & 0 & 481 \\
\hline Summe & 405 & 355 & 153 & 34 & 14 & 1 & 962 \\
\hline
\end{tabular}

Angaben N (\%)

Um die erhaltenen Benzodiazepin-und Z-Substanz Dosen vergleichbar zu machen, wurden Diazepam Äquivalenzdosen berechnet. Es zeigte sich kein signifikanter Unterschied bezüglich der eingenommenen Dosis von Benzodiazepinen $(p=0,08)$. Auch der Kontingenzkoeffizient nach Pearson zeigte mit einem Wert von 0,13 nur einen schwachen Zusammenhang für Dosissteigerung und Sturzrisiko an. Auffällig erscheint jedoch, dass höhere Dosen von mehr als 10mg Diazepam Äquivalent fast nur Patienten in der Sturzgruppe erhielten, auch wenn die absoluten Häufigkeiten eher gering ausfielen (s. Tabelle 30).

Tabelle 30 Benzodiazepin-Dosis als Diazepam-Äquivalente 


\begin{tabular}{|l|r|r|}
\hline Diazepam $0 \mathrm{mg}$ & $402(83,6)$ & $429(89,2)$ \\
\hline Diazepam 2,5 mg & $3(0,6)$ & $2(0,4)$ \\
\hline Diazepam $5 \mathrm{mg}$ & $12(2,6)$ & $13(2,7)$ \\
\hline Diazepam 7,5 mg & $1(0,2)$ & $1(0,2)$ \\
\hline Diazepam 10 mg & $46(9,6)$ & $34(7,1)$ \\
\hline Diazepam 15 mg & $8(1,7)$ & $2(0,4)$ \\
\hline Diazepam 20 mg & $5(1,0)$ & 0 \\
\hline Diazepam 25 mg & $1(0,2)$ & 0 \\
\hline Diazepam 27,5 mg & $1(0,2)$ & 0 \\
\hline Diazepam 50 mg & $2(0,4)$ & 0 \\
\hline Summe & 481 & 481 \\
\hline
\end{tabular}

Ein signifikanter Unterschied bezüglich der verabreichten Dosis zeigte sich bei den ZSubstanzen $(\mathrm{p}=0,02)$. Der Zusammenhang zwischen Dosissteigerung und Sturzrisiko war mit einem Kontingenzkoeffizienten nach Pearson von 0,08 jedoch ziemlich gering. Diese Inkongruenz der beiden Werte war darauf zurückzuführen, dass in beiden Gruppen keine große Variabilität in der Dosis verordneter Z-Substanzen bestand, dadurch blieb der Kontingenzkoeffizient niedrig. Im Dosisbereich $<5 \mathrm{mg}$ Diazepam finden sich jedoch höhere Einnahmezahlen im Sturzjahr 2013, so erklärt sich der signifikante p-Wert im WilcoxonTest (s. Tabelle 31).

Tabelle 31 Z-Substanz-Dosis als Diazepam-Äquivalente

\begin{tabular}{|l|r|r|}
\hline Z-Substanzen als Diazepam-Äquivalents Dosis & Sturzjahr 2013 N (\%) & Kontrollen 2013 N (\%) \\
\hline Diazepam 0 mg & $427(88,7)$ & $448(93,1)$ \\
\hline Diazepam 1,25 mg & $1(0,2)$ & 0 \\
\hline Diazepam 2,5 mg & $22(4,6)$ & $12(2,5)$ \\
\hline Diazepam 5 mg & $31(6,4)$ & $21(4,4)$ \\
\hline Diazepam 7,5 mg & 0 & 0 \\
\hline Diazepam 10 mg & 0 & 0 \\
\hline Summe & 481 & 481 \\
\hline
\end{tabular}

Wie bei den Benzodiazepinen und Z-Substanzen wurden auch für die verordneten Opiate Morphin Äquivalentdosen errechnet (s. Tabelle 32). Dabei zeigte sich kein signifikanter 
Unterschied $(p=0,3)$ bezüglich der verabreichten Morphindosen in beiden Gruppen. Der Kontingenzkoeffizient zeigte mit 0,18 ebenfalls nur einen schwachen Zusammenhang zwischen Dosissteigerung und Sturzrisiko an. Ein Dosisbezug für Opiate und Stürze konnte in Zusammenschau beider Werte somit nicht gezeigt werden.

Tabelle 32 Opiat-Dosis als Morphin-Äquivalente

\begin{tabular}{|l|r|r|}
\hline \multicolumn{1}{|c|}{ Opiate als Morphin-Äquivalente } & Sturz 2013 N (\%) & Kontrollen 2013 N (\%) \\
\hline Morphin 0 mg & $333(69,2)$ & $317(65,9)$ \\
\hline Morphin $5 \mathrm{mg}$ & $4(0,8)$ & $3(0,6)$ \\
\hline Morphin 7,5 mg & $8(1,7)$ & $3(0,6)$ \\
\hline Morphin $10 \mathrm{mg}$ & $18(3,7)$ & $17(3,5)$ \\
\hline Morphin $15 \mathrm{mg}$ & $72(15,0)$ & $105(21,8)$ \\
\hline Morphin $20 \mathrm{mg}$ & $11(2,3)$ & $9(1,9)$ \\
\hline Morphin $22,75 \mathrm{mg}$ & $1(0,2)$ & 0 \\
\hline Morphin $30 \mathrm{mg}$ & $20(4,2)$ & $8(1,7)$ \\
\hline Morphin $35 \mathrm{mg}$ & 0 & 0 \\
\hline Morphin $40 \mathrm{mg}$ & 0 & $1(0,2)$ \\
\hline
\end{tabular}




\begin{tabular}{|l|r|r|}
\hline Morphin $45 \mathrm{mg}$ & $1(0,2)$ & 0 \\
\hline Morphin $60 \mathrm{mg}$ & $4(0,8)$ & $10(2,1)$ \\
\hline Morphin $65 \mathrm{mg}$ & 0 & $1(0,2)$ \\
\hline Morphin $70 \mathrm{mg}$ & 0 & $1(0,2)$ \\
\hline Morphin $120 \mathrm{mg}$ & $1(0,2)$ & $3(0,6)$ \\
\hline Morphin $130 \mathrm{mg}$ & $3(0,6)$ & $1(0,2)$ \\
\hline Morphin $135 \mathrm{mg}$ & 0 & $2(0,4)$ \\
\hline Morphin $180 \mathrm{mg}$ & $2(0,4)$ & 0 \\
\hline Morphin $195 \mathrm{mg}$ & $2(0,4)$ & 0 \\
\hline Morphin $300 \mathrm{mg}$ & $1(0,2)$ & 0 \\
\hline Summe & 481 & 481 \\
\hline
\end{tabular}




\section{Diskussion}

\subsection{Zusammenfassung der Ergebnisse}

Im Evangelischen Krankenhaus Göttingen Weende stürzten im Jahr 2013805 und im Jahr 2014778 Patienten. Die meisten Stürze fanden sich in der geriatrischen Abteilung. Frauen stürzten häufiger als Männer. Der Anteil an Sturzpatienten lag in der Altersgruppe der 75bis 85-Jährigen am höchsten. Das mittlere Alter der gestürzten Patienten betrug 82 Jahre. Die Sturzinzidenz lag sowohl im Jahr 2013 als auch 2014 bei ca. 4 Stürzen pro 1000 Patiententage. In der geriatrischen Abteilung lag die Inzidenz bei 9,1 Stürzen pro 1000 Patiententagen, was der höchsten Inzidenzrate entsprach. Die Mehrheit der Patienten stürzte zu Tageszeit zwischen 6 und 22 Uhr. Mehrfachstürze fanden sich bei ca. $20 \%$ der Patienten. Die Sturzprotokolle als Dokumentationsinstrument für Stürze wurden in 90\% der Fälle vollständig ausgefüllt. Leichte Verletzungen konnten bei ca. 1/3 der Stürze beobachtet werden. Schwere Verletzungen fanden sich glücklicherweise nur bei 3,7\% aller Stürze. Zwei Patienten starben an den Folgen ihres Sturzes. Der Einfluss einzelner Variablen auf das Sturzrisiko wurde zunächst mit dem Chi-Square- bzw. Fisher's Exact-Test auf statistische Signifikanz geprüft. Variablen mit einem statistisch signifikanten Einfluss und ausreichender Beobachtungszahl wurden dann in ein finales Regressionsmodell überführt. So konnte eine Beeinflussung der Ergebnisse durch Störvariablen/Confounder reduziert werden. Signifikanten Einfluss auf das Sturzrisiko zeigten unter den psychoaktiven Medikamenten langwirksame Benzodiazepine $(\mathrm{OR}=3,49 ; \mathrm{KI}=1,16-10,52)$, SNRI $(\mathrm{OR}=2,57 ; \mathrm{KI}=1,23$ 5,12), Z-Substanzen $(\mathrm{OR}=2,29 ; \mathrm{KI}=1,38-3,59)$ und sedierende Neuroleptika $(\mathrm{OR}=1,87$; $K I=1,08-3,23)$. Leichten signifikanten Einfluss zeigten außerdem ACE-Hemmer und Sartane $(\mathrm{OR}=1,42 ; \mathrm{KI}=1,07-1,89)$. Digoxin $(\mathrm{OR}=0,32 ; \mathrm{KI}=0,11-0,99)$ und Aldosteronrezeptor-Antagonisten $(\mathrm{OR}=0,54 ; \mathrm{KI}=0,33-0,88)$ zeigten einen signifikant negativen, also das Sturzrisiko reduzierenden Einfluss. Morphinmedikation hatte keinen signifikanten Einfluss auf das Sturzrisiko. Eine Hyponatriämie und Leukozytose im Aufnahmelabor erhöhten ebenfalls das Risiko, während des Aufenthalt zu stürzen. Von den Nebendiagnosen hatten das Parkinson-Syndrom, Delir und Exsikkose einen signifikanten Einfluss. Patienten mit einem Delir hatten in dieser Studie das höchste Risiko zu stürzen. Bei den Hauptdiagnosen waren Neubildungen C00-D48 mit einem erhöhten und Krankheiten des Verdauungssystems K00-K93 mit einem erniedrigten Risiko für Stürze assoziiert. Allgemein zeigte sich 
eine gute Modellanpassung an die Daten ( $\mathrm{p}=0,89$; Hosmer-Lemeshow-Test). Für die Vitalparameter am Sturztag, den BMI sowie die geriatrischen Assessments konnten aufgrund fehlender Dokumentation in den Akten lediglich univariate OR berechnet werden. Dabei ergab sich für einen erhöhten Blutdruck vor dem Sturz, einen BMI unter 17,5 und Werte von unter 20 Punkten im Tinetti-Test ein signifikanter Einfluss auf die Erhöhung des Sturzrisikos. Sturz- und Kontrollgruppe unterschieden sich signifikant in der Anzahl gleichzeitig eingenommener psychoaktiver Medikamente. Ein signifikanter Unterschied zeigte sich auch bei der verabreichten Dosis von Z-Substanzen.

\subsection{Confounding in Fall-Kontrollstudien}

Fall-Kontrollstudien sind eine Form der retrospektiven Beobachtungsstudien (Grimes und Schulz 2005). Deren Ergebnisse sollten im Idealfall durch interne Validität gekennzeichnet sein. Das bedeutet, dass die Ausprägungen der abhängigen Variable durch die unabhängige Variable erklärt werden können (Wacholder 1995; Newman 2013). Allerdings wird diese Beziehung mitunter durch einen sogenannten Confounder, auch Störvariable oder Störfaktor genannt, beeinflusst. Eine Variante von Confounding das Confounding by Indication gilt in Beobachtungsstudien als größte Limitation (Psaty et al. 1999; Skelly et al. 2012). Wird beispielsweise ein Benzodiazepin aufgrund eines Verwirrtheitszustandes (Indikation) verabreicht, so kann es sein, dass der Verwirrtheitszustand und nicht die Medikation mit einem Benzodiazepin den Effekt auf das Sturzereignis beeinflusst. Oft wird zwischen den sehr ähnlichen Begriffen Mediator und Confounder unterschieden (MacKinnon 2000). Bei der sogenannten Mediatorvariablen wird ein kausaler Effekt zwischen der abhängigen und der unabhängigen Variablen durch den Mediator vermittelt. Oft wird hierbei das Beispiel angeführt, bei dem Koffein Lungenkrebs verursacht, der Effekt jedoch auf die Zigarette zum Kaffee zurückzuführen ist (Tang et al. 2010; Guertin et al. 2016). Im Rahmen von Beobachtungsstudien spricht man jedoch eher von Confoundern als von Mediatoren, bei denen ein kausaler Effekt nicht zwingend erforderlich sein muss. Es ist sogar möglich, dass mehrere Confounder gleichzeitig einen Effekt auf die unabhängige Variable haben (Cleophas und Zwinderman 2007). Es ist somit erforderlich für wichtige Confounder zu kontrollieren. „Matching“ (Zuordnen) nach wichtigen personenbezogenen Parametern, wie Alter und Geschlecht, sowie die Anwendung verschiedener Regressionsverfahren sind dabei die wichtigsten Optionen (Greenland 2008; Rose und van der Laan 2009). In der hier vorliegenden Studie wurden die Patienten der Kontrollgruppe nach den Parametern Alter, Geschlecht und Abteilungszugehörigkeit den Patienten der Sturzgruppe zugeordnet. Das Alter kann als wichtige Störvariable fungieren, da ältere Patienten ohnehin anfälliger für 
Stürze sind und die Zunahme von Multimorbidität im Alter, sowie die damit einhergehende Polypharmazie ebenfalls Risikofaktoren für Stürze darstellen (Chiu et al. 2015; Richardson et al. 2015). Geschlechtsspezifische Unterschiede, wie unterschiedliche Gebrechlichkeit von Mann und Frau im Alter, könnten die Ergebnisse ebenfalls beeinflussen (Hitcho et al. 2004; Kinne und Klewer 2016). Abteilungszugehörigkeit ist kein typischer „Matchingparameter“. Jedoch spielen abteilungsspezifische Krankheitsbilder eine Rolle im Sturzgeschehen und können somit zumindest teilweise kontrolliert werden (Greenland 2008). Allerdings kann auch das Matching an sich zu Bias (Verzerrungen) führen, weshalb die Matching-Variablen zumindest in die finale statistische Analyse miteinbezogen werden sollten (Greenland 2008; Pearce 2016). Nicht kontrollierte, also nicht berücksichtige Confounder, sollten anschließend in den Limitationen der Studie herausgestellt werden (Skelly et al. 2012).

\subsection{Stärken und Schwächen}

Eine Stärke der Studie war die große Zahl von 1583 in die Studie eingeschlossener Stürze, von denen 962 für die Auswertung genutzt wurden. Die Daten der Sturzpatienten wurden über einen klar definierten Zeitraum von zwei Jahren erhoben. Dabei wurden detaillierte Informationen über viele verschiedene Sturzvariablen gesammelt. Die Medikation wurde in einem Zeitintervall von 24 Stunden vor dem Sturzereignis erfasst. So konnte ein Bezug zum Sturzzeitpunkt hergestellt werden.

In anderen Studien dieses Projekts war es zu Problemen mit Doppelerfassungen gekommen, daher wurde für jeden Patienten kontrolliert, ob es bei Verlegungen in andere Abteilungen und der damit verbundenen Neuvergabe der Patientennummer zu neuen Stürzen gekommen war. So konnte verhindert werden, dass ein erneuter Sturz eines Patienten auf einer anderen Station dazu führte, dass er scheinbar als neuer Sturzpatient erfasst wurde. Um Verzerrungen der Ergebnisse durch wichtige Störfaktoren wie Alter, Geschlecht und Abteilungszugehörigkeit zu vermeiden, wurden die Patienten in der Kontrollgruppe nach genau diesen Parametern den Sturzpatienten zugeordnet.

Da pro Patient nur ein Sturz in die Auswertung einbezogen und der Sturz mit der negativeren Sturzfolge ausgewählt wurde, könnte die Häufigkeit von Sturzverletzungen leicht überschätzt werden. Für die Sturzgruppe des Jahres 2014 wurde keine Kontrollgruppe erhoben, somit können für diesen Zeitraum keine Aussagen zum Sturzrisiko einzelner Variablen getroffen werden. 
Zudem konnten viele Variablen aufgrund mangelhafter oder fehlender Dokumentation in den Arztbriefen und Patientenakten nur teilweise erhoben werden. Risikobestimmungen für diese Variablen sind somit nur in Form von univariaten Odds-Ratios möglich und somit sehr anfällig für Störeffekte. Das bedeutet, dass die Ergebnisse unter dem Einfluss einer Störvariable stehen, die eigentlich für den beobachteten Effekt verantwortlich ist. Es lässt sich somit nur eine Tendenz beobachten. Dies galt insbesondere auch für die geriatrischen Assessments, die nur in der Geriatrie ausreichend dokumentiert wurden. Einige in der Vorbereitung der Arbeit als wichtig eingestufte Variablen, wie beispielweise die Polyneuropathie, konnten aus diesem Grund gar nicht erhoben werden und könnten so eine Rolle als Störvariable spielen.

Ferner wurde die eingenommene Anzahl an Psychopharmaka erfasst und zwischen Sturzund Kontrollgruppe verglichen. Dabei konnte lediglich ein signifikanter Unterschied gezeigt werden. Ab welcher Anzahl eingenommener Psychopharmaka Patienten tatsächlich gefährdet sind zu stürzen und welche Kombination am risikoreichsten ist, konnte mit der angewendeten Methodik nicht gezeigt werden. Genauso verhält es sich beim Vergleich der berechneten Äquivalenzdosen für Benzodiazepine, Z-Substanzen und Opiate.

Darüber hinaus wurden nicht alle eingenommenen Medikamente erfasst. So entsteht ein weiterer möglicher Störeffekt, da Substanzklassen wie Diuretika oder NSAR nicht berücksichtig wurden, obwohl sie in einigen Studien einen Effekt auf das Sturzrisiko hatten (Leipzig et al. 1999b; Frels et al. 2002; Walker et al. 2005).

Letztlich kann auch die Generalisierbarkeit der Ergebnisse hinterfragt werden, da die Erhebung der Stürze ausschließlich im Evangelischen Krankenhaus Göttingen-Weende durchgeführt wurde. Faktoren wie Personalschlüssel, Ausstattung der Patientenzimmer, Beleuchtung, verschiedene Bodenbeläge und Verordnungsgewohnheiten innerhalb der Abteilungen können sich bei Krankenhäusern unterscheiden. Erhebungen zu Sturzrisikofaktoren sollten daher in weiteren regionalen Krankenhäusern durchgeführt werden und mit den Ergebnissen dieser Studie verglichen werden. 


\subsection{Medikamente und medizinische Diagnosen als Risikofaktoren für Stürze, eine kritische Auseinandersetzung im Kontext unerwünschter Arzneimittelnebenwirkungen- und Interaktionen}

In diesem Kapitel sollen die Medikamente, die als Risikofaktor für Stürze identifiziert wurden, mit bisherigen Studienergebnisse verglichen und im Kontext ihrer Indikation, ihrer unerwünschten Nebenwirkungen und möglicher Arzneimittelinteraktionen kritisch beleuchtet werden. Anschließend soll auf das Problem der Polypharmazie im Alter eingegangen und eine Mehrfachmedikation mit psychoaktiven Medikamenten als eigenständiger Risikofaktor etabliert werden.

\subsubsection{Benzodiazepine und Z-Substanzen}

Schlafstörungen finden sich sehr häufig bei alten Menschen (Foley et al. 1995; Leitlinie Nicht erholsamer Schlaf 2017). Im Krankenhaus ist mehr als jeder dritte ältere Patient betroffen (Isaia et al. 2011). Unruhezustände und Agitation sind weitere sehr häufig anzutreffende Symptome bei geriatrischen hospitalisierten Patienten (Fong et al. 2009). Zur Therapie werden oft Medikamente eingesetzt, die das Risiko für Stürze erhöhen können. Zur pharmakologischen Therapie von Schlafstörungen werden Benzodiazepine, ZSubstanzen, Antidepressiva, sedierende Neuroleptika und pflanzliche Präparate empfohlen (Leitlinie Nicht erholsamer Schlaf 2017). Bei Unruhezuständen, wie dem Delir, kommen Neuroleptika und Benzodiazepine zum Einsatz (Lorenzl et al. 2012).

Langwirksame Benzodiazepine hatten in der hier vorliegenden Studie den größten Einfluss $(\mathrm{OR}=3,49)$ aller verabreichten Medikamente auf das Sturzrisiko, allerdings bei geringen Verordnungshäufigkeiten (s. Tabelle 21). Auch in zahlreichen anderen Studien wurde für diese Substanzgruppe ein erhöhtes Sturzrisiko nachgewiesen (Leipzig et al. 1999a; Wang et al. 2001a; Cumming und Le Couteur 2003; Hartikainen et al. 2007). Langsamere Anflutung und langer Wirkungsspiegel sind typisch für diese Substanzklasse und erklären das höhere Abhängigkeitspotenzial (Llorente et al. 2000). Bei abrupter Beendigung der Einnahme kann es zu Entzugssymptomen kommen. Diese treten jedoch schneller bei den kurzwirksamen Substanzen auf (Pétursson 1994). Darüber hinaus treten paradoxe Reaktionen bei der Behandlung mit dieser Substanzklasse auf. Ca. 1\% aller mit Benzodiazepinen behandelten Patienten entwickeln Symptome wie Aggressivität, motorische Unruhe und Logorrhö. Gerade ältere Patienten erscheinen gefährdet (Mancuso et al. 2004). Die häufigsten unerwünschten Nebenwirkungen, die vermutlich das Sturzrisiko erhöhen, sind Sedierung, Mus- 
kelrelaxation und Konzentrationsstörungen. (Uzun et al. 2010). Kurzwirksame Benzodiazepine waren mit 68 Verordnungen $(14,2 \%)$ nach Mirtazapin die an den zweithäufigsten verordneten psychoaktiven Medikamente in dieser Studie (s. Tabelle 33). Ein signifikanter Einfluss auf das Sturzrisiko konnte nicht gezeigt werden $(\mathrm{p}=0,12)$. Viele andere Studien wiesen hingegen für die kurzwirksamen Präparate eine signifikante Assoziation mit Stürzen nach (Leipzig et al. 1999a; Passaro et al. 2000; Wang et al. 2001a; Leipzig et al. 1999a; Walker et al. 2005; Shuto et al. 2010). Die kurzwirksamen Präparate werden in der kurzfristigen Behandlung der Insomnie auch bei älteren Menschen empfohlen (Leitlinie Nicht erholsamer Schlaf 2017). Da Insomnie jedoch meist chronisch auftritt, sollte das Konzept der kurzfristigen Behandlung generell überdacht werden.

Auch die zahlreichen Medikamentenlisten, die sich damit beschäftigen, welche Medikamente bei älteren Menschen vermieden oder eingesetzt werden sollten, sind bei den Benzodiazepinen inkonsistent. Beers Criteria und FORTA-Klassifikation lehnen die Behandlung mit Benzodiazepinen bei älteren Menschen ab. Die Ausnahme stellen epileptische Anfälle dar, bei denen Benzodiazepine im akuten Anfall nach wie vor das Medikament der 1. Wahl sind. Die PRISCUS -Liste hingegen gestattet den Einsatz von kurz- und mittellang wirksamen Benzodiazepinen in geringer Dosis. Valide vergleichende Studien zur Häufigkeit von unerwünschten Nebenwirkungen bei der Behandlung älterer Patienten mit BenzodiazepinPräparaten unter dem Aspekt der Halbwertszeit sind nicht existent und sollten in Zukunft durchgeführt werden.

Bezüglich der Inzidenz von unerwünschten Nebenwirkungen erscheinen insbesondere Kombinationen von Benzodiazepinen mit anderen psychoaktiven Medikamenten, insbesondere Z-Substanzen, Antidepressiva oder Neuroleptika problematisch. Die komplexen Interaktionsmechanismen im ZNS aufgrund der Pharmakodynamik und Pharmakokinetik sowie des Einflusses auf den Metabolismus anderer Medikamente sind dann nicht mehr zu überblicken. Die häufigsten Kombinationen innerhalb der Sturzgruppe waren SSRI mit tetrazyklischen Antidepressiva (15 Verordnungen, 3,1\%) und tetrazyklische Antidepressiva mit Benzodiazepinen 14 Verordnungen, 2,9\%, s. Tabelle 34). Bedenklich erscheint auch eine Doppelmedikation mit zwei Benzodiazepinen oder ein Benzodiazepin mit einer ZSubstanz, da es so zu einer Wirkungsverstärkung am GABA-A-Rezeptor kommen kann (Gunja 2013). Diese Kombinationen waren glücklicherweise nur selten zu finden (Benzodiazepin und Benzodiazepin in Sturzgruppe $\mathrm{N}=4,0,83 \%$ und $\mathrm{N}=0,0 \%$ in der Kontrollgruppe; Benzodiazepin und Z-Substanz N=8, 1,66\% in der Sturzgruppe und N=2, 0,42\% in der Kontrollgruppe, s. Tabelle 34). 
Neben den lang wirksamen Benzodiazepinen konnte auch für die bisher eher weniger gut untersuchten Z-Substanzen ein erhöhtes Sturzrisiko $(\mathrm{OR}=2,29 ; \mathrm{p}=0,01)$ nachgewiesen werden. Z-Substanzen galten - aufgrund der pharmakologischen Unterschiede - lange als die bessere Alternative zu Benzodiazepinen. In klinischen Studien zeigten sich allerdings ähnliche Probleme in Bezug auf kognitive- und motorische Einschränkungen (Gunja 2013). Auch das Risiko für Stürze konnte bereits in einigen Studien belegt werden (Rhalimi et al. 2009; Cashin und Yang 2011; Chang et al. 2011). Auch für die Dosis verabreichter ZSubstanzen konnte gezeigt werden, dass höhere Dosen signifikant häufiger in der Sturzgruppe verabreicht wurden $(\mathrm{p}=0,02)$. Zolpidem ist fast vollständig an Plasmaproteine gebunden und wird über die Leber mit Cytochrom-Enzymen, vor allem Cyp3A4, eliminiert. Ältere Menschen zeigen bei gleicher verabreichter Dosis im Vergleich zu jüngeren höhere Plasma-Spiegel und eine verlängerte Halbwertszeit (Barkin 2007; Gunja 2013). Unerwünschte Nebenwirkungen wie Amnesie oder visuelle Halluzinationen sind insbesondere bei höheren Dosierungen eine mögliche Folge und könnten Stürze begünstigen (Gunja 2013).

\subsubsection{Antidepressiva und Neuroleptika}

Mirtazapin, ein tetrazyklisches Antidepressivum, gilt als nebenwirkungsarm und wird nach den Kriterien der PRISCUS -Liste zur Behandlung von Schlafstörungen und Depressionen im Alter empfohlen (Holt et al. 2010; Alam et al. 2013). Mirtazapin war das am meisten verordnete psychoaktive Medikament sowohl in der Sturz- als auch in der Kontrollgruppe. Eine Assoziation mit Stürzen konnte, nach Kontrolle für verschiedene Störvariablen, in der adjustierten Analyse nicht nachgewiesen werden. Bei der Sturzgruppe im Jahr 2014 fanden sich deutlich mehr Verordnungen gegenüber dem Jahr 2013. Leider wurde keine Kontrollgruppe für das Sturzjahr 2014 ausgewertet, sodass keine valide Aussage zu möglichen Unterschieden bei der Verordnung getroffen werden kann.

Ferner ließ sich für Antidepressiva der Klasse SNRI ein signifikant höheres Sturzrisiko nachweisen. Dies deckt sich mit vergleichbaren Studienergebnissen (Woolcott et al. 2009; Park et al. 2015). SSRI und SNRI sind sich von ihrem Nebenwirkungsprofil sehr ähnlich. Als unerwünschte Nebenwirkungen werden unter anderem Übelkeit, Obstipation, Diarrhoe, Hyponatriämie, Blutungsneigung im GI-Trakt, sexuelle Funktionsstörungen, psychotische Reaktionen und Schlafstörungen genannt (Ferguson 2001, Benkert und Regen 2014). Mit Ausnahme der seltenen psychotischen Reaktionen und der Hyponatriämie fanden sich keine Symptome, die unmittelbar mit Stürzen in Verbindung stehen (s. Tabelle 22 und 23). 
Darüber hinaus sind die multiplen pharmakologischen Wechselwirkungen problematisch. Wichtige hepatische Cytochrom-Enzyme beim Stoffwechsel von Antidepressiva und Neuroleptika sind die Klassen CYP3A4, CYP2D6, CYP2C9 und CYP2C19 (Messiha 1993; Lane 1996; Urichuk et al. 2008; Sansone und Sansone 2014). Da Beta-Blocker wie Metoprolol und Carvedilol ebenfalls über diese Enzyme abgebaut werden, kann es zu einer verminderten Ausscheidung mit Wirkungsverstärkung und daraus resultierenden Problemen bei der Blutdruckregulation kommen.

Die Datenlage zum Sturzrisiko bei der Einnahme von Neuroleptika ist, wie eingangs beschrieben, inkonsistent (Leipzig et al. 1999a; Krauss et al. 2005; Hartikainen et al. 2007; Chiu et al. 2015). Einige Studien wiesen ein erhöhtes Sturzrisiko nach. Dabei wurde allerdings nicht zwischen typischen oder atypischen Neuroleptika bzw. dem antipsychotischem Potenzial der einzelnen Präparate unterschieden (Kallin et al. 2004; Chiu et al. 2015). In der PRISCUS -Liste werden hochpotent, antipsychotisch wirksame Präparate eher abgelehnt und sedierende, niedrigpotent antipsychotisch wirksame Präparate wie beispielsweise Melperon empfohlen (Holt et al. 2010).

Neuroleptika werden im stationären Rahmen zur Behandlung von Delir und psychotischen Syndromen z.B. bei Demenz eingesetzt (Wehling 2012; Lorenzl et al. 2012, Leitlinie Demenzen 2016). Immer häufiger finden sie Anwendung ohne die zugelassenen Indikationen als sogenannter „Off-Label-Use“ ( Alexander et al. 2011; Nissen et al. 2014). Eine Zulassung zur kurzfristigen Behandlung bei Schlafstörungen existiert nur für Melperon und Pipamperon (Carton et al. 2015; Leitlinie Nicht erholsamer Schlaf 2017).

In dieser Studie fand sich ein erhöhtes Sturzrisiko für sedierende Neuroleptika. Für die klassischen hochpotent antipsychotisch wirksamen Präparate fand sich keine Assoziation mit Stürzen. Mögliche Ursachen für die Erhöhung des Sturzrisikos unter Therapie mit sedierenden Neuroleptika könnten ähnlich wie bei den Antidepressiva diverse Nebenwirkungen und Arzneimittelinteraktionen sein. Zu den Nebenwirkungen zählen Hypotension, Müdigkeit, anticholinerge Effekte, Transaminasenanstieg und Hyponatriämie (Nissen et al. 2014). Insbesondere anticholinerge Wirkungen, Hypotension und Hyponatriämie könnten im Zusammenhang mit Stürzen von Bedeutung sein. Interaktionen finden sich bei einer Vielzahl von Medikamenten. Explizit werden SSRI, SNRI, Antikonvulsiva wie Carbamazepin und Antihypertensiva genannt (ZumBrunnen und Jann 1998; Urichuk et al. 2008). Aufgrund der geringen Anzahl von Studien zum Sturzrisiko unter Einnahme von Neuroleptika sollten zukünftig weitere Untersuchungen der verschiedenen Substanzklassen durchgeführt werden. 


\subsubsection{Herz- und Kreislauf-Medikation}

Aus der Gruppe der Antihypertensiva zeigten ACE-Hemmer und Sartane einen signifikanten Einfluss auf das Sturzrisiko $(\mathrm{OR}=1,42 ; \mathrm{p}=0,03)$. Beide Substanzklassen gelten als nebenwirkungsarm und weder ACE-Hemmer noch Sartane sind bislang mit Stürzen in Verbindung gebracht worden. Jedoch könnte ein als unerwünschte Nebenwirkung beschriebener Blutdruckabfall unter maximal ausdosierter Therapie mit ACE-Hemmern Stürze begünstigen. Eventuell sind die Ergebnisse auch durch Störfaktoren beeinflusst. Sowohl in der Therapie des arteriellen Blutdrucks und der Herzinsuffizienz werden ACE-Hemmer als First-Line-Medikamente eingesetzt (Leitlinie Bluthochdruck 2014 und Herzinsuffizienz 2016). Ein erhöhter Blutdruck zeigte sich in der univariaten Analyse als Risikofaktor. Auch Gangavati et al. konnten in ihrer Studie zeigen, dass ein unkontrolliert erhöhter Blutdruck signifikant mit Stürzen assoziiert war (Gangavati et al. 2011). Allerdings ist auch anzumerken, dass ACE-Hemmer den zerebralen Blutfluss steigern und so im Falle einer orthostatischen Hypotension eher protektiv wirken müssten (Lipsitz et al. 2005). Confounding durch die Einnahme mehrerer Antihypertensiva ist möglich (Tinetti et al. 2014).

Ein signifikant protektiver Effekt konnte für Aldosteronrezeptor-Antagonisten und Digoxin gezeigt werden. Spironolacton und Eplerenon werden zur Behandlung der Herzinsuffizienz eingesetzt (Leitlinie Herzinsuffizienz 2016) Die Diagnose Herzinsuffizienz fand sich bei ca. jedem dritten Patienten, sowohl in Sturz als auch in der Kontrollgruppe und ist somit ein häufiges Krankheitsbild im Alter (s. Tabelle 35). Eine Verbindung mit Stürzen wurde nicht beobachtet. Symptome der Herzinsuffizienz wie Dyspnoe, eingeschränkte Belastungsfähigkeit, Schwäche, Erschöpfbarkeit, Müdigkeit, Gedächtnisstörungen, Verwirrtheitszustände stellen allerdings mögliche Risikofaktoren für Stürze dar (Oliver et al. 1997; Hendrich et al. 2003, Hill et al. 2007). Eine positive Beeinflussung der Symptome durch Aldosteronrezeptor-Antagonisten scheint möglich. Allerdings könnten auch andere Diuretika, die in dieser Studie nicht untersucht wurden, mögliche Störfaktoren darstellen und die Ergebnisse beeinflusst haben.

Ein zu den bisherigen Studienergebnissen konträrer Befund fand sich für das Herzglykosid Digoxin. Digoxin findet Anwendung bei der chronischen Herzinsuffizenz mit Vorhofflimmern (Leitlinie Herzinsuffizienz 2016). Digoxin reduziert bei ambulanter Anwendung die Hospitalisierungsrate, zeigte in amerikanischen Studien jedoch auch eine erhöhte Mortalität (The Digitalis Investigation Group 1997). Daher werden Verschreibungen inzwischen kritisch betrachtet (Vamos et al. 2015; Ziff et al. 2015). Leipzig et al. (1999b) fanden für Digoxin-Präparate eine leichte Assoziation mit Stürzen (Leipzig et al. 1999b). Allerdings 
ist festzustellen, dass dabei vorwiegend Studien aus dem ambulanten Bereich einbezogen werden. Das erforderliche Dosis-Monitoring ist im Krankenhaus engmaschiger möglich, was zur Sicherheit in der Anwendung beiträgt. Auch in den verschiedenen PIM-Listen besteht Uneinigkeit. Die PRISCUS-Liste lehnt den Einsatz von Digoxin streng ab und empfiehlt andere Antiarrhythmika. Die FORTA-Klassifikation hingegen teilt Digoxin der Kategorie B zu. Die Wirksamkeit ist demnach erwiesen, es bestehen jedoch Einwände bezüglich der Sicherheit. Da sich das Anwendungsgebiet für Digoxin immer weiter einschränkt, könnte sich eine zukünftige Evaluation schwierig gestalten.

\subsubsection{Medizinische Diagnosen}

Da die Hyponatriämie unter Einnahme von Antidepressiva und Neuroleptika gehäuft auftritt, soll an dieser Stelle auf ihre Bedeutung bei Stürzen eingegangen werden. Eine Hyponatriämie konnte in mehreren Studien im Zusammenhang mit Stürzen nachgewiesen werden (Renneboog et al. 2006; Moret et al. 2008; Gankam Kengne et al. 2008)). Auch in der vorliegenden Untersuchung war eine Hyponatriämie im Aufnahmelabor signifikant häufiger mit Stürzen assoziiert, als ein normaler Natriumspiegel im Plasma. Die Hyponatriämie ist im Alter ein häufiger Zustand, da die Fähigkeit der Niere, den Urin zu konzentrieren und Natrium zurück zu resorbieren, nachlässt. Hinzu kommt die häufige Therapie mit Diuretika, Laxanzien oder Psychopharmaka (Morley 2015). Durch Einnahme von SNRI, SSRI oder Neuroleptika kommt es sekundär vermutlich zum Syndrom der inadäquaten ADH-Sekretion (Moret et al. 2008). Durch die erhöhte ADH-Sekretion kommt es zur Wasserretention und Absinken des Natriumspiegels. Im Verlauf entwickelt sich durch die Wasserausscheidung der Niere wieder eine Euvolämie bei bestehender Hyponatriämie. Vergesslichkeit, eingeschränkte Aufmerksamkeit, orthostatische Dysregulation, Kopfschmerzen, Lethargie, Krämpfe, Delir, Erbrechen, zerebrale Krämpfe und Ateminsuffizienz sind Symptome, die im Rahmen einer Hyponatriämie auftreten können. Viele dieser Symptome sind bekannte Risikofaktoren für Stürze (Salgado et al. 1994; Hill et al. 2007; Morley 2007; Moret et al. 2008; Jansen et al. 2015). Renneboog et al. konnten zeigen, dass Patienten mit Hyponatriämie signifikant häufiger eine verminderte Aufmerksamkeit und motorische Defizite aufwiesen. Die Testergebnisse bei Hyponatriämie waren vergleichbar mit einem moderaten Alkoholkonsum (Renneboog et al. 2006).

Wichtig ist es, die in dieser Studie ebenfalls signifikant mit Stürzen in Verbindung stehende Exsikkose $(\mathrm{OR}=1,85 ; \mathrm{p}=0,0002)$ in diesen Kontext einzubeziehen. Exsikkose ist eine häufige klinische Diagnose, deren Ätiologie vielfältig sein kann. Es existieren iso-, hypo- und hypertone Formen mit einhergehender Hypo- oder Hypernatriämie. Überwiegend finden 
sich allerdings Formen mit Hyponatriämie. Die Exsikkose ist außerdem eine häufige Ursache für das Delir (Vasilevskis et al. 2012; Morley 2015; Mazur et al. 2016). Interessanterweise hatten Delir, Exsikkose und Hyponatriämie unabhängig voneinander, also kontrolliert für gegenseitiges Confounding, ein erhöhtes Sturzrisiko.

Auch eine Leukozytose im Aufnahmelabor wurde signifikant häufiger $(O R=1,40 ; p=0,02)$ bei Sturzpatienten gefunden (s. Tabelle 23). Die Leukozytose spielt in der Akutdiagnostik einer Infektion bei älteren Patienten eher eine untergeordnete Rolle (Mouton et al. 2001; Rowe und Juthani-Mehta 2013). Typische Infekt Symptome fehlen oft, was einen verzögerten Behandlungsbeginn mit steigender Morbidität und Letalität zur Folge hat (Werner und Kuntsche 2000). Unspezifische Symptome wie Schwäche und Somnolenz könnten Stürze begünstigen (Gavazzi und Krause 2002; Rowe und Juthani-Mehta 2013; Hepper et al. 2013). Allerdings könnte auch eine begonnene Antibiotikatherapie mit den vielfältigen Arzneimittelinteraktionen einen möglichen Störfaktor darstellen (Joos 1998; Russo et al. 2002). Kurioserweise konnte ein signifikant niedrigeres Sturzrisiko bei Patienten mit Infektionen des Verdauungstrakts in der Hauptdiagnose gefunden werden (s. Tabelle 25). Eine erhöhte pflegerische Zuwendung aufgrund gesteigerter Stuhlfrequenz im Rahmen der Infektion könnte die Verringerung des Sturzrisikos bewirken.

Bösartige Neubildungen zeigten als einzige Hauptdiagnose eine signifikante Verbindung zum Sturzrisiko. Onkologische Erkrankungen sind eine typische Erkrankung des geriatrischen Patienten (Hansen 1998). Zwar werden Stürze oft im Zusammenhang mit onkologischen Diagnosen erwähnt, die Prävalenz für Stürze unter onkologischen geriatrischen Patienten liegt in den meisten Studien jedoch nur gering über der für nicht onkologische geriatrische Patienten (Goodridge und Marr 2002; Allan-Gibbs 2010; Wildes et al. 2015). Symptome wie Fatigue, Schmerz und Depression finden sich häufig bei onkologischen Patienten (Rao und Cohen 2004). Erschöpfung, Müdigkeit und Kraftlosigkeit im Rahmen des Fatigue-Syndroms könnten Stürze begünstigen. Die in der Tumortherapie eingesetzten Chemotherapeutika könnten mit ihren vielfältigen unerwünschten Nebenwirkungen und Arzneimittelinteraktionen in diesem Zusammenhang einen möglichen nicht kontrollierten Störfaktor darstellen (van Leeuwen et al. 2011).

\subsubsection{Polypharmazie}

Polypharmazie und die daraus resultierenden Interaktionsmechanismen stellen einen eigenen Risikofaktor für Stürze dar ( Hammond und Wilson 2013; Chiu et al. 2015; Park et al. 2015; Richardson et al. 2015). Generell gilt, dass ab einer Medikation mit mehr als 4 ver- 
schiedenen Arzneimitteln unerwünschte Arzneimittelnebenwirkungen signifikant häufiger auftreten (Carbonin et al. 1991; Vora et al. 2011). Wie bereits aufgeführt, nimmt mehr als die Hälfte aller stationären geriatrischen Patienten mehr als 5 Medikamente ein (Nobili et al. 2011; Rambhade et al. 2012). Medikamente, die als potenziell sicher eingestuft werden, könnten durch Interaktionen in ihrer Pharmakokinetik-und dynamik beeinflusst werden und zu einem erhöhten Risiko für UAW und Stürze führen. Mitunter ist sogar eine vitale Gefährdung möglich (Weiner et al. 1998; Rambhade et al. 2012; Mosshammer et al. 2016).

In dieser Studie konnte gezeigt werden, dass in der Sturzgruppe signifikant mehr Psychopharmaka eingenommen wurden $(\mathrm{p}<0,001)$. Polymedikation mit psychoaktiven Medikamenten stellt einen in der Literatur bisher wenig beachteten Risikofaktor für Stürze dar (Kukreja et al. 2013). Der Einsatz von mehreren Substanzen z.B. SSRI und Benzodiazepine bei der Behandlung von Depressionen oder Schlafstörungen wird kontrovers diskutiert und es sollte gerade bei älteren Patienten genau abgewogen werden, in wie weit die Patienten von einer solchen Therapie überhaupt profitieren (Furukawa et al. 2001; Dunlop und Davis 2008). Interventionsstudien mit Dosisreduktionsmaßnahmen und systematischem Absetzen von Medikamenten, die auch das Sturzrisiko mit einbeziehen, sollten in Zukunft vermehrt durchgeführt werden. Dabei sollte der Fokus nicht nur auf Stürze, sondern auch auf Vigilanz und Mobilitätsparameter gelegt werden.

Zusammenfassend lässt sich festhalten, dass zahlreiche Medikamente eine Assoziation mit Stürzen aufwiesen. Die gegenwärtige Verschreibungspraktik sollte im Hinblick auf Polymedikation und Arzneimittelinteraktionen hinterfragt und in weiterführenden Studien unter den Aspekten Nebenwirkungen und Stürze untersucht werden. Dabei könnte auch auf Therapiekonflikte, die bei einer leitliniengerechten Behandlung multimorbider geriatrischer Patienten entstehen, eingegangen werden. Alternative Therapieoptionen wie die Schlafrestriktion zur Verbesserung der Schlafhygiene oder psychoedukative Maßnahmen sollten in randomisierten kontrollierten Studien getestet werden. Derartige alternative Maßnahmen erfordern natürlich auch zusätzliche personelle Ressourcen, die mit Blick auf die derzeitige gesundheitspolitische Diskussion um einen erhöhten Pflegebedarf schwierig zu realisieren wären. Als Lösung könnten, wie auf Intensivstationen als Sitzwachen bereits etabliert, auch studentische „Sturzwachen“ bei agitierten Patienten auf Normalstationen eingesetzt werden.

Bei klarer Indikationsstellung in der kurzfristigen Behandlung von Delir oder Epilepsie und in der langfristigen Behandlung der Depression werden einige psychoaktive Medikamente weiterhin ihren Platz haben. Dabei sollten die individuelle Medikamentendosis, Dosisan- 
passung im Verlauf, Komorbiditäten und Arzneimittelwechselwirkungen streng beachtet werden. Arzneimittel-Apps, wie das bereits im Evangelischen Krankenhaus GöttingenWeende eingesetzte RpDoc der RpDoc Solutions GmbH, die den Anwender vor möglichen Arzneimittelinteraktionen warnen, sollten weiter optimiert werden, um den Einsatz im klinischen Alltag praktikabler zu machen.

\subsection{Wie können Sturzpatienten identifiziert werden und sind geriatrische Assessments zur Sturzrisikoerfassung sinnvoll?}

Um Stürze im Krankenhaus zu reduzieren und gefährdete Patienten rechtzeitig zu erfassen, sind verschiedene Sturzrisikoerfassungssysteme, sogenannte Sturzrisikoassessments, entwickelt worden. Ein solches Assessment soll einfach durchzuführen und auf Basis möglichst einfach zu erhebender Variablen schnell eine Einstufung der Patienten ermöglichen. In Abhängigkeit von der ermittelten Risikoklasse sollen dann individuelle Sturzvermeidungsmaßnahmen ergriffen werden. Die am häufigsten angewandten Scores sind das Stratify Fall Risk Assessment Tool und die Morse-Fall Scale. Beide Scores beinhalten sehr ähnliche Variablen: Sturz in der Vorgeschichte, Mobilitätseinschränkung und Orientierung finden sich in beiden Assessments. Bei der Morse-Fall Scale kommt noch Multimorbidät und beim Stratify Tool Agitation und Toilettenfrequenz hinzu. Auch im Evangelischen Krankenhaus Göttingen-Weende existiert ein Sturzrisikoerfassungsbogen. Dieser bezieht die gleichen erwähnten Variablen ein. Viele Risikoscores sind nicht validiert und haben Probleme bezüglich ihrer Inter-Rater-Reliablität. Bei Validierung des Stratify Tools fanden sich eine relativ niedrige Sensitivität und Spezfität (Oliver et al. 2008). Bei der Morse-Fall Scale verhielt es sich ähnlich (Oliver et al. 2004; Schwendimann et al. 2006a; Chow et al. 2007). Die Morse-Fall Scale scheint aufgrund der höheren Spezifität eher geeignet zu sein, nicht gefährdete Patienten zu identifizieren. Problematisch sind vor allem die geringen Werte im positiv prädiktiven Wert, mit Schwankungen zwischen 12 und $30 \%$. Aufgrund dieser Beobachtungen wird von der Anwendung von den Risikoassessments abgeraten (Oliver et al. 2004; Schwendimann et al. 2006b; Chow et al. 2007; Oliver et al. 2008). Allerdings bestehen auch Zweifel bei der Wirksamkeit von getroffenen Sturzvermeidungsmaßnahmen. Schwendimann et al. konnten in ihrer über 4 Jahre durchgeführten Studie mit Implementierung eines Sturzvermeidungsprogramms keinen Effekt auf Sturzfrequenz und die Reduzierung von Sturzverletzungen beobachten (Schwendimann et al. 2006b). In einer anderen randomisierten kontrollierten Studie, konnte zwar gezeigt werden, dass Interventionen wie 
Mobilitäts- und Orientierungstraining die Patienten physisch verbesserten, ein Rückgang der Sturzfrequenz und der Verletzungsrate wurde aber nicht beobachtet (Lord et al. 2005). Der Erfolg von Sturzvermeidungsscreening und Vermeidungsmaßnahmen erscheint vor diesem Hintergrund fraglich (Oliver et al. 2000; Lundin-Olsson et al. 2003; Lord et al. 2005; Park 2018).

In der hier vorliegenden Studie wurde kein Sturzrisikoassessment-Werkzeug validiert. Sofern durchgeführt und dokumentiert, wurden die in der geriatrischen Abteilung erhobenen Assessments in der Sturz- und Kontrollgruppe ausgewertet. Geriatrische Assessments liefern in ihrer Zusammenschau, im Gegensatz zum traditionellen Fokus auf Krankheitssymptomen und Krankengeschichten, ein mehrdimensionales Bild des Patienten. So können psychologische, soziale, kognitive und funktionelle Einschränkungen bei der optimalen und individuellen Behandlungsplanung Berücksichtigung finden (Rosen und Reuben 2011). Wie bereits eingangs erwähnt, weisen die meisten Assessments keine hohe Sensitivität in der Vorhersage von Stürzen auf und sind deshalb zur Risikoeinstufung einzelner Patienten eher ungeeignet. Die relativ guten Werte bezüglich der Spezifität könnten zumindest geeignet sein, festzustellen, welchem Patienten keine Hilfs- und Sturzvermeidungsmaßnahmen zukommen müssen. Unsere Ergebnisse bestätigen die beschriebene Problematik. Kein Assessment mit Ausnahme des Tinetti-Tests (POMA) zeigte in den verschiedenen Wertebereichen signifikante Assoziation mit Stürzen. Dies ist konform mit bisherigen Studienergebnissen (Trueblood et al. 2001; Köpke und Meyer 2006; Kegelmeyer et al. 2007; Barry et al. 2014). Allerdings muss angemerkt werden, dass unterschiedliche Cut-Off-Werte Einfluss auf Sensitivität und Spezifität haben und somit die Studienergebnisse beeinflussen. Eine Vergleichbarkeit von Studien wird dadurch erschwert (Köpke und Meyer 2006). Darüber hinaus ist die zu vergebende Punktzahl für die einzelnen Teilbereiche von der Interpretation der den Test durchführenden Person abhängig. Unterschiedliche Personen vergeben eventuell andere Punktzahlen (Inter-Rater-Reliabilität).

Ein weiteres Problem sind die teilweise sehr geringen Beobachtungszahlen. Der Timed-Upand-Go-Test wurde lediglich bei 50 Personen in der Sturzgruppe und 83 Patienten in der Kontrollgruppe durchgeführt. Signifikante Unterschiede wurden nicht beobachtet. Ein möglicher Grund für die geringe Zahl an Tests könnte sein, dass nur relativ rüstige Patienten für diesen Test ausgewählt worden sind. Die am stärksten gefährdeten mobilitätseingeschränkten Patienten wurden eventuell gar nicht berücksichtigt. Allerdings gelang es auch anderen Studien nicht, eine Verbindung mit Stürzen herzustellen (Beauchet et al. 2011; Stone et al. 2012; Barry et al. 2014). 
Außerdem ist in der hier vorliegenden Studie nicht genau bekannt, zu welchem Zeitpunkt des Klinikaufenthalts die Assessments durchgeführt worden sind. Der Tinetti/Poma-Test ist Grundlage für die physiotherapeutische Therapieplanung und wird daher routinemäßig bei Aufnahme erhoben. Auch der Barthel-Index ist wichtig zur Verlaufsbeobachtung in der geriatrischen Rehabilitation und wird daher einmal zu Anfang des Aufenthalts und einmal am Ende durchgeführt. Zwischenzeitliche Änderungen des Gesundheitszustands wurden dann eventuell gar nicht erfasst. Es stellt sich auch die Frage nach der Änderungssensitivität der Assessment-Untersuchungen, inwieweit die Tests überhaupt in der Lage sind, Änderungen des Gesundheitszustandes zu erfassen (Pardasaney et al. 2012).

Ferner konnte gezeigt werden, dass nicht nur Mobilitätseinschränkungen für ein erhöhtes Sturzrisiko verantwortlich sind, sondern auch die kognitiven Fähigkeiten in der multidimensionalen Genese von Stürzen eine wichtige Rolle einnehmen. Während andere Untersuchungen gezeigt haben, dass niedrige Punktzahlen im MMST mit einem erhöhten Sturzrisiko verbunden waren, fand sich dafür in der hier vorliegenden Studie kein Hinweis (Ramirez et al. 2010). Da der MMST im Rahmen der Demenzdiagnostik eingesetzt wird und Demenz als Diagnose auch keinen Einfluss $(\mathrm{OR}=1,29 ; \mathrm{p}=0,13)$ auf das Sturzrisiko zeigte, ist dies wenig überraschend (Foy et al. 1995; Zhang et al. 2016). Da der Test für die Diagnostik von Demenzerkrankungen entwickelt wurde, ist er nicht dafür validiert, sensitiv kognitive Einschränkungen unter der Therapie mit psychoaktiven Substanzen zu erfassen. Gegebenenfalls könnte hierfür ein neuer Test entwickelt und validiert werden.

Es gilt also festzuhalten, dass die meisten Assessments eher ungeeignet sind, sturzgefährdete Patienten zu identifizieren. Dies liegt wohl zum einen an der Eindimensionalität einiger Assessments und zum anderen an den vielfältigen intrinsischen und extrinsischen Ursachen für Stürze. So existieren vielfältige Störgrößen, die die Ergebnisse bei dem für die Fragestellung nicht optimal gewählten Studiendesign einer Fall-Kontrollstudie beeinflusst haben könnten. Zur Prüfung der Validität sollten eher prospektive Studien durchgeführt werden. Die geriatrischen Assessments behalten natürlich trotzdem ihren Stellenwert in der ganzheitlichen Beurteilung der Patienten. Generell sollten allgemeine Sturzvermeidungsmaßnahmen gefördert werden. Dazu zählt wie bereits beschrieben der Ausbau des Pflegeschlüssels und das Schaffen einer patientenfreundlichen Umgebung, um Verletzungsrisiken zu reduzieren. Generell sollten auch physiotherapeutische Maßnahmen zur Verbesserung der Mobilität im Alter gefördert werden, um so die Partizipation am gesellschaftlichen Leben zu erhalten. 


\subsection{Schlussfolgerung}

Die vorliegende Studie liefert Informationen über Sturzrisikofaktoren in einem deutschen Krankenhaus der Grund- und Regelversorgung. Auf Basis von Sturzprotokollen konnten Zahlen zur Inzidenz und Häufigkeit von Stürzen sowie sturzassoziierten Verletzungen ermittelt werden. Durch Nutzung ausgewählter statistischer Methoden und unter Kontrolle für wichtige Störfaktoren konnten Risikofaktoren für Stürze ermittelt werden. Die meisten Stürze fanden sich erwartungsgemäß in der geriatrischen Abteilung. Die meisten der bereits in bisherigen Studien ermittelten Risikofaktoren fanden sich auch in der hier vorliegenden Untersuchung. Teilweise wurden jedoch auch kontroverse Ergebnisse beobachtet. So fand sich eine Reduzierung des Sturzrisikos bei Behandlung mit dem PRISCUS-gelisteten Medikament Digoxin und den bei Herzinsuffizienz verabreichten AldosteronrezeptorAntagonisten (s. Tabelle 22). Eventuell könnte eine positive Beeinflussung der Herzinsuffizienz zu diesem Effekt beigetragen haben. Darüber hinaus bietet die Studie einen Dosisbezug für wichtige Medikamente wie Benzodiazepine, Z-Substanzen und Opiate (s. Tabelle 30-32). Allerdings konnten dabei nur bei den Z-Substanzen höhere Dosen mit einem erhöhten Sturzrisiko in Verbindung gebracht werden. An dieser Stelle würden sich weiterführende Studien anbieten. Insbesondere Polypharmazie und die gleichzeitige Gabe mehrerer Psychopharmaka erhöhten das Risiko für Stürze und Arzneimittelwechselwirkungen. Polypharmazie wurde bereits häufig mit Stürzen in Verbindung gebracht (Chiu et al. 2015; Richardson et al. 2015). Dass allerdings auch die - wie in der vorliegenden Studie belegt gleichzeitige Einnahme verschiedener ZNS-wirksamer Präparate das Sturzrisiko erhöht, konnte zwar vermutet werden, wurde aber in bisherigen Studien noch nicht als Risikofaktor identifiziert. Zukünftig sollten vermehrt, wie bereits in einer ersten Pilotstudie geschehen, klinische randomisierte kontrollierte Studien durchgeführt werden, die prüfen, welche Kombinationen risikoärmer sind und ob überhaupt ein klinischer Nutzen bei der Therapie mit mehreren Präparaten entsteht (Michalek et al. 2014). Alternative Therapiemaßnahmen könnten im Vergleich zur bisherigen medikamentösen Therapie bei Insomnie im Hinblick auf Stürze, kognitive Fähigkeiten und Mobilität untersucht werden. Zudem könnten Computer gestützte Systeme zur Vermeidung von Arzneimittelinteraktionen in der klinischen Praxis validiert werden. 


\section{$6 \quad$ Zusammenfassung}

Hintergrund: Stürze im Alter sind häufig. Sturzraten schwanken zwischen 1,6 und 17 Stürzen auf 1000 Behandlungstage. In den geriatrischen Abteilungen finden sich die höchsten Sturzraten. Bei der Genese von Stürzen existieren viele intrinsische und extrinsische Risikofaktoren. Zu den extrinsischen iatrogenen Risikofaktoren werden einige Medikamente, insbesondere Sedativa, gezählt. Durch Beeinflussung von Kognition und Mobilität erhöhen einige Substanzen das Risiko zu stürzen. Polypharmazie und die damit einhergehenden UAW spielen in diesem Zusammenhang ebenfalls eine wichtige Rolle. Krankenhausund länderspezifische Unterschiede in der Patientenversorgung machen eine eigene Evaluierung der Risikofaktoren erforderlich.

Fragestellung und Ziel: Sturzhäufigkeit und sturzassoziierte Risikofaktoren sollen in dieser Studie ermittelt werden. Darüber hinaus sollen auch Patienten-bezogene Parameter wie die geriatrischen Assessments im Kontext der Sturzprävention und Sturzrisikoerfassung beurteilt und evaluiert werden.

Methoden: Retrospektive Auswertung aller Sturzprotokolle der Jahre 2013 und 2014 von Patienten mit einem Alter $\geq 65$ Jahre. Kontrollgruppe zugeordnet nach den Variablen Alter, Geschlecht und Abteilungszugehörigkeit. Die Risikofaktoren der 481 gestürzten Patienten im Jahr 2013 und 481 Patienten der Kontrollgruppe 2013 wurden mit univariater- und multivariater logistischer Regressionsanalyse zur Bestimmung der Odds-Ratios ausgewertet. Die kollektiven Mittelwertunterschiede werden mit Hilfe eines t-Tests für abhängige Stichproben verglichen.

\section{Ergebnisse:}

Die Sturzinzidenz betrug 4,36 (2013) und 4,46 (2014) auf 1000 Behandlungstage. Es stürzten mehr Frauen (58,8\% 2013 und 52,8\% 2014) als Männer (41,2\% 2013 47,2\% 2014) Leichte Verletzungen waren mit 30,7\% relativ häufig, schwere Verletzungen mit 3,7\% und 1,7\% Operationsbedürftigkeit eher selten. Die Sturzprotokolle waren zu 88,6\% vollständig ausgefüllt worden. Lang wirksame Benzodiazepine hatten unter den Medikamenten den stärksten Einfluss auf das Sturzrisiko $(\mathrm{OR}=3,49 ; \mathrm{KI}=1,16-10,52)$. Signifikanten Einfluss zeigten außerdem SNRI (OR=2,57; KI=1,23-5,12), Z-Substanzen (OR=2,29; KI=1,383,59), sedierende Neuroleptika ( $\mathrm{OR}=1,87 ; \mathrm{KI}=1,08-3,23)$ und ACE-Hemmer/Sartane 
$(\mathrm{OR}=1,42 ; \mathrm{KI}=1,07-1,89)$. Digoxin $(\mathrm{OR}=0,32 ; \mathrm{KI}=0,11-0,99)$ und AldosteronrezeptorAntagonisten $(\mathrm{OR}=0,54 ; \mathrm{KI}=0,33-0,88)$ hatten einen signifikant Sturz-reduzierenden Einfluss. Unter den Laborwerten waren Hyponatriämie ( $\mathrm{OR}=1,52$; $\mathrm{KI}=1,15-2,02)$ und Leukozytose $(\mathrm{OR}=1,39 ; \mathrm{KI}=1,05-1,87)$ signifikant häufiger mit Stürzen assoziiert. Neubildungen $(\mathrm{OR}=1,35 ; \mathrm{KI}=1,13-3,01)$ waren als einzige Hauptdiagnose, Delir $(\mathrm{OR}=3,74 ; \mathrm{KI}=2,26$ 6,21), Exsikkose (OR=1,85; KI=1,28-2,67) und Parkinson-Syndrom $(\mathrm{OR}=2,38 ; \mathrm{KI}=1,27$ 4,46) als Nebendiagnosen signifikant häufiger in der Sturzgruppe zu finden. Krankheiten des Verdauungssystems (OR=0,49; KI=0,29-0,81) hatten einen das Sturzrisiko verringernden Einfluss. Allein bei den Z-Substanzen konnte ein signifikanter Dosisunterschied $(p=0,02)$ nachgewiesen werden. Sturz- und Kontrollgruppe unterschieden sich signifikant in der Anzahl eingenommener psychoaktiver Medikamente $(\mathrm{p}<0,001)$. Von den geriatrischen Assessments zeigte lediglich der Tinetti-Test (POMA) in univariater Analyse $(\mathrm{OR}=2,27 ; \mathrm{KI}=0,97-5,3)$ eine signifikante Assoziation mit Stürzen.

\section{Diskussion und Schlussfolgerung:}

In dieser Studie konnte für einige psychoaktive Medikamente eine signifikante Assoziation mit Stürzen nachgewiesen werden. Darunter fielen auch die bisher weniger gut untersuchten neueren Medikamente wie Z-Substanzen und Antidepressiva der Klasse SNRI. Für die gerade in der Geriatrie häufig verordneten tetrazyklischen Antidepressiva konnte keine Assoziation mit Stürzen nachgewiesen werden. Unter dem Aspekt von Arzneimittelnebenwirkungen sind viele ZNS-wirksame Medikamente insbesondere bei gleichzeitiger Anwendung kritisch zu betrachten. Durch ihre Wirkung auf die kognitiven Fähigkeiten und auch auf die Mobilität führen einige Medikamente zu einer Zunahme des Sturzrisikos. Es fanden sich auch im Vergleich mit bisherigen Studien kontroverse Ergebnisse. So konnte eine signifikant häufigere Verschreibung in der Kontrollgruppe für Digoxin und Aldosteronrezeptor-Antagonisten beobachtet werden. Gerade Digoxin wird in der Anwendung bei geriatrischen Patienten sehr kritisch betrachtet und wird in der PRISCUS-Liste nicht empfohlen. Darüber hinaus konnte eine signifikant häufigere Mehrfachmedikation mit Psychopharma$\mathrm{ka}$ in der Sturzgruppe beobachtet werden. Unter dem Aspekt der Polypharmazie im Alter und damit einhergehenden vielfältigen UAW und gegenseitigen Arzneimittelinteraktionen sind solche Therapieregime kritisch zu bewerten. In diesem Zusammenhang sollten alternative Maßnahmen bei der Behandlung von Depression und Insomnie im Vergleich zur medikamentösen Therapie im Hinblick auf Kognition und Mobilität, in randomisiertkontrollierten Studien verglichen werden. Die geriatrischen Assessments waren mit Ausnahme des Tinetti-Tests nicht in der Lage, sturzgefährdete Risikopatienten zu ermitteln. 


\section{$7 \quad$ Anhang}

Tabelle 33 Häufigkeiten der verordneten Medikamente

\begin{tabular}{|c|c|c|c|}
\hline Psychoaktive Medikamente & Sturzjahr $2013^{*}$ & Kontrollen $2013^{* *}$ & Sturzjahr $2014 *$ \\
\hline \multicolumn{4}{|c|}{ Kurz- und mittellang wirksame Benzodiazepine } \\
\hline Lorazepam & 40 & 13 & 42 \\
\hline Lormetazepam & 23 & 31 & 24 \\
\hline Alprazolam & 0 & 1 & 0 \\
\hline Brotizolam & 1 & 0 & 0 \\
\hline Temazepam & 0 & 1 & 0 \\
\hline Midazolam & 1 & 0 & 0 \\
\hline Oxazepam & 4 & 6 & 12 \\
\hline \multicolumn{4}{|l|}{ Langwirksame Benzodiazepine } \\
\hline Bromazepam & 4 & 0 & 8 \\
\hline Nitrazepam & 3 & 0 & 1 \\
\hline Tetrazepam & 1 & 0 & 0 \\
\hline Diazepam & 2 & 2 & 5 \\
\hline Flunitrazepam & 2 & 0 & 0 \\
\hline Clonazepam & 1 & 2 & 1 \\
\hline Dikaliumclorazepat & 1 & 1 & 1 \\
\hline \multicolumn{4}{|l|}{ Neuroleptika antipsychotisch } \\
\hline Quetiapin & 20 & 18 & 17 \\
\hline Haloperidol & 9 & 2 & 8 \\
\hline Risperidon & 6 & 10 & 10 \\
\hline Olanzapin & 5 & 1 & 4 \\
\hline Clozapin & 0 & 0 & 1 \\
\hline \multicolumn{4}{|l|}{ Neuroleptika sedierend } \\
\hline Melperon & 38 & 19 & 38 \\
\hline Prothipendyl & 6 & 7 & 7 \\
\hline Promethazin & 0 & 0 & 1 \\
\hline \multicolumn{4}{|l|}{ Neuroleptika andere } \\
\hline Tiaprid & 0 & 1 & 0 \\
\hline
\end{tabular}




\begin{tabular}{|c|c|c|c|}
\hline \multicolumn{4}{|l|}{ SNRI } \\
\hline Duloxetin & 14 & 10 & 22 \\
\hline Venlafaxin & 13 & 4 & 13 \\
\hline \multicolumn{4}{|l|}{ SSRI } \\
\hline Citalopram & 48 & 32 & 33 \\
\hline Escitalopram & 1 & 4 & 2 \\
\hline Fluoxetin & 1 & 1 & 3 \\
\hline Paroxetin & 0 & 0 & 1 \\
\hline \multicolumn{4}{|c|}{ Tetrazyklische Antidepressiva } \\
\hline Mirtazapin & 83 & 61 & 127 \\
\hline Maprotilin & 0 & 1 & 0 \\
\hline \multicolumn{4}{|c|}{ Trizyklische Antidepressiva } \\
\hline Amitriptylin & 8 & 8 & 16 \\
\hline Doxepin & 6 & 3 & 1 \\
\hline Trimipramin & 2 & 2 & 0 \\
\hline Opipramol & 3 & 1 & 6 \\
\hline \multicolumn{4}{|l|}{ Z-Substanz } \\
\hline Zopiclon & 21 & 19 & 19 \\
\hline Zolpidem & 34 & 14 & 38 \\
\hline \multicolumn{4}{|c|}{ andere Sedativa } \\
\hline Baldrian & 30 & 32 & 26 \\
\hline Agomelatin & 0 & 0 & 1 \\
\hline Melatonin & 0 & 0 & 1 \\
\hline \multicolumn{4}{|c|}{ Antikonvulsiva } \\
\hline Pregabalin & 22 & 19 & 18 \\
\hline Gabapentin & 10 & 8 & 7 \\
\hline Valproinsäure & 0 & 2 & 2 \\
\hline Lamotrigin & 2 & 1 & 4 \\
\hline Carbamazepin & 2 & 2 & 4 \\
\hline Levetiracetam & 0 & 10 & 10 \\
\hline Oxcarbazepin & 1 & 0 & 0 \\
\hline \multicolumn{4}{|c|}{ Antihypertensive Medikation } \\
\hline \multicolumn{4}{|c|}{ ACE-Hemmer/Sartane } \\
\hline Ramipril & 162 & 156 & 175 \\
\hline Enalapril & 51 & 37 & 40 \\
\hline Lisinopril & 11 & 20 & 18 \\
\hline Candesartan & 32 & 14 & 41 \\
\hline Eprosartan & 1 & 0 & 0 \\
\hline
\end{tabular}




\begin{tabular}{|c|c|c|c|}
\hline Irbesartan & 1 & 0 & 2 \\
\hline Losartan & 14 & 10 & 7 \\
\hline Olmesartan & 4 & 1 & 1 \\
\hline Telmisartan & 2 & 0 & 3 \\
\hline Valsartan & 30 & 37 & 22 \\
\hline \multicolumn{4}{|l|}{ B-Blocker } \\
\hline Metoprolol & 159 & 154 & 156 \\
\hline Bisoprolol & 103 & 96 & 116 \\
\hline Carvedilol & 2 & 2 & 6 \\
\hline Nebivolol & 33 & 34 & 27 \\
\hline Atenolol & 1 & 0 & 2 \\
\hline Propanolol & 2 & 1 & 3 \\
\hline Sotalol & 0 & 1 & 1 \\
\hline \multicolumn{4}{|c|}{ Kalziumantagonisten } \\
\hline Lercanidipin & 43 & 22 & 42 \\
\hline Amlodipin & 74 & 92 & 58 \\
\hline Nitrendipin & 2 & 1 & 2 \\
\hline Felodipin & 3 & 1 & 1 \\
\hline \multicolumn{4}{|c|}{ Alosteronrezeptorantagonisten } \\
\hline Spironolacton & 31 & 50 & 36 \\
\hline Eplerenon & 2 & 4 & 1 \\
\hline \multicolumn{4}{|c|}{ Alpha-2-Agonisten/Alpha-1-Antagonisten (nach PRISCUS-Liste fraglich) } \\
\hline Moxonidin & 21 & 18 & 12 \\
\hline Urapidil & 1 & 0 & 1 \\
\hline \multicolumn{4}{|c|}{ Antihypertensiva nach PRISCUS-Liste nicht empfohlen } \\
\hline Doxazosin & 2 & 6 & 4 \\
\hline Clonidin & 0 & 0 & 1 \\
\hline Isosorbidmononitrat & 0 & 1 & 0 \\
\hline Nifedipin & 1 & 0 & 0 \\
\hline \multicolumn{4}{|c|}{ Antiarrhythmika } \\
\hline \multicolumn{4}{|c|}{ Antiarrhythmika nach PRISCUS-Liste empfohlen } \\
\hline Digitoxin & 16 & 22 & 17 \\
\hline Amiodaron & 20 & 5 & 20 \\
\hline Diltiazem & 1 & 2 & 1 \\
\hline Verapamil & 3 & 4 & 2 \\
\hline \multicolumn{4}{|c|}{ Antiarrhythmika nach PRISCUS-Liste nicht empfohlen } \\
\hline Digoxin & 4 & 17 & 6 \\
\hline
\end{tabular}




\begin{tabular}{|l|r|r|r|}
\hline Buprenorphin & 1 & 0 & 0 \\
\hline Tilidin & 27 & 23 & 25 \\
\hline Fentanyl & 8 & 9 & 9 \\
\hline Oxycodon & 93 & 110 & 105 \\
\hline Hydromorphon & 13 & 10 & 16 \\
\hline Palladon & 0 & 2 & 0 \\
\hline Piritramid & 4 & 4 & 8 \\
\hline Tramadol & 3 & 5 & 7 \\
\hline Morphin & 2 & 5 & 3 \\
\hline
\end{tabular}

* Erhalt bis $24 \mathrm{~h}$ vor Sturz, ${ }^{* *}$ Erhalt bis $24 \mathrm{~h}$ vor Tag 5 nach Aufnahme.

Tabelle 34 Kombinationen eingenommener Psychopharmaka Sturzjahr 2013

\begin{tabular}{|c|c|c|c|c|}
\hline $\begin{array}{c}\text { Kombinationen Psychoaktiver } \\
\text { Medikamente }\end{array}$ & $\mathbf{N}$ & $\%$ & univariate OR (95KI) & $\mathbf{p}$ \\
\hline \multicolumn{5}{|c|}{ Benzodiazepin kurz- und mittellang und lang-wirksam } \\
\hline Sturz & 4 & 0,8 & $1,06(0,99-1,01)$ & 0,12 \\
\hline Kein Sturz & 0 & 0 & & \\
\hline \multicolumn{5}{|c|}{ Benzodiazepin und tetrazyklisches Antidepressivum } \\
\hline Sturz & 14 & 2,9 & $1,77(0,73-4,26)$ & 0,19 \\
\hline Kein Sturz & 8 & 1,7 & & \\
\hline \multicolumn{5}{|l|}{ Benzodiazepin und Z-Substanz } \\
\hline Sturz & 8 & 1,7 & $4,05(0,86-19,17)$ & 0,06 \\
\hline Kein Sturz & 2 & 0,4 & & \\
\hline \multicolumn{5}{|c|}{ tetrazyklisches Antidepressivum und Z-Substanz } \\
\hline Sturz & 11 & 2,3 & $1,38(0,55-3,47)$ & 0,48 \\
\hline Kein Sturz & 8 & 1,7 & & \\
\hline \multicolumn{5}{|c|}{ tetrazyklisches Antidepressivum und sedierendes Neuroleptikum } \\
\hline Sturz & 11 & 2,3 & $11,23(1,45-87,36)$ & 0,01 \\
\hline Kein Sturz & 1 & 0,2 & & \\
\hline \multicolumn{5}{|c|}{ Z-Substanz und sedierendes Neuroleptikum } \\
\hline Sturz & 2 & 0,4 & $1,04(0,24-104,86)$ & 0,5 \\
\hline Kein Sturz & 0 & 0 & & \\
\hline \multicolumn{5}{|c|}{ Benzodiazepin und sedierendes Neuroleptikum } \\
\hline Sturz & 11 & 2,3 & $3,72(1,03-13,45)$ & 0,03 \\
\hline Kein Sturz & 3 & 0,6 & & \\
\hline \multicolumn{5}{|l|}{ SSRI und Z-Substanz } \\
\hline Sturz & 3 & 0,6 & $1,5(0,25-9,04)$ & 1 \\
\hline
\end{tabular}




\begin{tabular}{|l|r|r|r|l|}
\hline Kein Sturz & 2 & 0,4 & & \\
\hline
\end{tabular}

\begin{tabular}{|c|c|c|c|c|}
\hline \multicolumn{5}{|c|}{ SSRI und Benzodiazepin } \\
\hline Sturz & 9 & 1,9 & $1,81(0,61-5,46)$ & 0,28 \\
\hline Kein Sturz & 5 & 1,0 & & \\
\hline \multicolumn{5}{|c|}{ SSRI und tetrazyklisches Antidepressivum } \\
\hline Sturz & 15 & 3,1 & $1,37(0,63-3,03)$ & 0,42 \\
\hline Kein Sturz & 11 & 2,3 & & \\
\hline \multicolumn{5}{|c|}{ SSRI und sedierendes Neuroleptikum } \\
\hline Sturz & 2 & 0,4 & $0,66(0,11-3,98)$ & 1 \\
\hline Kein Sturz & 3 & 0,6 & & \\
\hline \multicolumn{5}{|c|}{ SNRI und Z-Substanz } \\
\hline Sturz & 1 & 0,2 & $1,01(1-1,01)$ & 1 \\
\hline Kein Sturz & 0 & 0 & & \\
\hline \multicolumn{5}{|c|}{ SNRI und Benzodiazepin } \\
\hline Sturz & 3 & 0,6 & $1,50(0,25-9,04)$ & 1 \\
\hline Kein Sturz & 2 & 0,4 & & \\
\hline \multicolumn{5}{|c|}{ SNRI und tetrazyklisches Antidepressivum } \\
\hline Sturz & 8 & 1,7 & $2,01(0,60-6,74)$ & 0,25 \\
\hline Kein Sturz & 4 & 0,8 & & \\
\hline \multicolumn{5}{|c|}{ SNRI und sedierendes Neuroleptikum } \\
\hline Sturz & 3 & 0,6 & $1,01(1-1,01)$ & 1 \\
\hline Kein Sturz & 0 & 0 & & \\
\hline
\end{tabular}

Tabelle 35 Univariate Odds-Ratios aller Einflussvariablen 2013

\begin{tabular}{|c|c|c|c|c|}
\hline Einflussvariablen & $\mathbf{N}$ & $\%$ & univariate OR (95KI) & $\mathbf{p}$ \\
\hline \multicolumn{5}{|c|}{ Benzodiazepine kurz- und mittellang wirksam } \\
\hline Sturz & 68 & 14,1 & $1,36(0,92-2,0)$ & 0,12 \\
\hline Kein Sturz & 52 & 10,8 & & \\
\hline \multicolumn{5}{|c|}{ Benzodiazepine lang wirksam } \\
\hline Sturz & 14 & 1,9 & $2,85(1,02-7,99)$ & 0,04 \\
\hline Kein Sturz & 5 & 1,0 & & \\
\hline
\end{tabular}




\begin{tabular}{|l|r|r|r|r|}
\hline Sturz & 40 & 8,3 & $1,32(0,81-2,14)$ & 0,27 \\
\hline Kein Sturz & 31 & 6,4 & & \\
\hline
\end{tabular}

\begin{tabular}{|c|c|c|c|c|}
\hline \multicolumn{5}{|c|}{ Neuroleptika sedierend } \\
\hline Sturz & 44 & 9,2 & $1,76(1,07-2,91)$ & 0,03 \\
\hline Kein Sturz & 26 & 5,4 & & \\
\hline \multicolumn{5}{|c|}{ Neuroleptika andere } \\
\hline Sturz & 1 & 0,2 & 0 & 1 \\
\hline Kein Sturz & 0 & & & \\
\hline \multicolumn{5}{|l|}{ SNRI } \\
\hline Sturz & 27 & 5,6 & $1,98(1,03-3,83)$ & 0,04 \\
\hline Kein Sturz & 14 & 2,9 & & \\
\hline \multicolumn{5}{|l|}{ SSRI } \\
\hline Sturz & 50 & 10,4 & $1,39(0,89-2,17)$ & 0,14 \\
\hline Kein Sturz & 37 & 7,7 & & \\
\hline \multicolumn{5}{|c|}{ Trizyklische Antidepressiva } \\
\hline Sturz & 19 & 4,0 & $1,37(0,68-2,77)$ & 0,38 \\
\hline Kein Sturz & 14 & 2,9 & & \\
\hline \multicolumn{5}{|c|}{ Tetrazyklische Antidepressiva } \\
\hline Sturz & 83 & 17,3 & $1,44(1,01-2,05)$ & 0,05 \\
\hline Kein Sturz & 61 & 12,7 & & \\
\hline \multicolumn{5}{|c|}{ Z-Substanz } \\
\hline Sturz & 55 & 11,4 & $1,75(1,12-2,75)$ & 0,01 \\
\hline Kein Sturz & 33 & 6,9 & & \\
\hline \multicolumn{5}{|c|}{ andere Sedativa } \\
\hline Sturz & 30 & 6,2 & $0,93(0,56-1,56)$ & 0,79 \\
\hline Kein Sturz & 32 & 6,7 & & \\
\hline \multicolumn{5}{|c|}{ Antikonvulsiva } \\
\hline Sturz & 37 & 7,7 & $0,87(0,55-1,38)$ & 0,56 \\
\hline Kein Sturz & 42 & 8,7 & & \\
\hline \multicolumn{5}{|c|}{ ACE-Hemmer/Sartane } \\
\hline Sturz & 308 & 64,0 & $1,33(1,03-1,73)$ & 0,03 \\
\hline Kein Sturz & 275 & 57,2 & & \\
\hline \multicolumn{5}{|c|}{ Beta-Blocker } \\
\hline Sturz & 300 & 62,4 & $1,11(0,86-1,44)$ & 0,43 \\
\hline Kein Sturz & 288 & 59,9 & & \\
\hline \multicolumn{5}{|c|}{ Kalzium-Antagonisten } \\
\hline Sturz & 122 & 25,4 & $1,07(0,80-1,43)$ & 0,65 \\
\hline
\end{tabular}




\begin{tabular}{|c|c|c|c|c|}
\hline \multicolumn{5}{|c|}{ Aldosteronrezeptor-Antagonisten } \\
\hline Sturz & 33 & 6,9 & $0,58(0,37-0,92)$ & 0,02 \\
\hline Kein Sturz & 54 & 11,2 & & \\
\hline \multicolumn{5}{|c|}{ Alpha-2-Agonisten/Alpha-1-Antagonisten } \\
\hline Sturz & 22 & 4,6 & $1,23(0,65-2,33)$ & 0,52 \\
\hline Kein Sturz & 18 & 3,7 & & \\
\hline \multicolumn{5}{|c|}{ Antihypertensiva nach PRISCUS-Liste nicht empfohlen } \\
\hline Sturz & 3 & 0,6 & $0,42(0,11-1,65)$ & 0,2 \\
\hline Kein Sturz & 7 & 1,5 & & \\
\hline \multicolumn{5}{|c|}{ Antiarrhythmika nach PRISCUS-Liste empfohlen } \\
\hline Sturz & 40 & 8,3 & $1,23(0,76-1,99)$ & 0,39 \\
\hline Kein Sturz & 33 & 6,9 & & \\
\hline \multicolumn{5}{|c|}{ Antiarrhythmika nach PRISCUS-Liste nicht empfohlen } \\
\hline Sturz & 4 & 0,4 & $0,22(0,08-0,68)$ & 0,004 \\
\hline Kein Sturz & 17 & 3,5 & & \\
\hline \multicolumn{5}{|l|}{ Opiate } \\
\hline Sturz & 151 & 31,4 & $0,85(0,65-1,12)$ & 0,24 \\
\hline Kein Sturz & 168 & 34,9 & & \\
\hline \multicolumn{5}{|c|}{ Nebendiagnosen } \\
\hline \multicolumn{5}{|c|}{ Parkinsonsyndrom } \\
\hline Sturz & 36 & 7,5 & $2,08(1,16-3,72)$ & 0,01 \\
\hline Kein Sturz & 18 & 3,7 & & \\
\hline \multicolumn{5}{|c|}{ Diabetes mellitus } \\
\hline Sturz & 131 & 27,3 & $1,17(0,88-1,57)$ & 0,27 \\
\hline Kein Sturz & 116 & 24,2 & & \\
\hline \multicolumn{5}{|l|}{ KHK } \\
\hline Sturz & 133 & 27,7 & $1,03(0,77-1,37)$ & 0,82 \\
\hline Kein Sturz & 130 & 27,0 & & \\
\hline \multicolumn{5}{|c|}{ Herzinsuffizienz } \\
\hline Sturz & 148 & 30,8 & $1,09(0,83-1,44)$ & 0,53 \\
\hline Kein Sturz & 139 & 29,0 & & \\
\hline \multicolumn{5}{|l|}{ Demenz } \\
\hline Sturz & 95 & 19,8 & $1,29(0,93-1,80)$ & 0,13 \\
\hline Kein Sturz & 77 & 16,0 & & \\
\hline
\end{tabular}




\begin{tabular}{|l|r|r|r|r|}
\hline \multicolumn{6}{|l|}{ Ischämischer Schlaganfall } \\
\hline Sturz & 92 & 19,1 & $1,25(0,89-1,73)$ & 0,2 \\
\hline Kein Sturz & 77 & 16,0 & & \\
\hline
\end{tabular}

\begin{tabular}{|c|c|c|c|c|}
\hline \multicolumn{5}{|l|}{ Epilepsie } \\
\hline Sturz & 24 & 5,0 & $1,21(0,66-2,22)$ & 0,54 \\
\hline Kein Sturz & 20 & 4,2 & & \\
\hline \multicolumn{5}{|l|}{ Exsikkose } \\
\hline Sturz & 108 & 22,5 & $1,92(1,37-2,7)$ & 0,0001 \\
\hline Kein Sturz & 63 & 13,0 & & \\
\hline \multicolumn{5}{|l|}{ Delir } \\
\hline Sturz & 81 & 16,9 & $4,03(2,49-6,53)$ & 0,0001 \\
\hline Kein Sturz & 23 & 4,8 & & \\
\hline \multicolumn{5}{|c|}{ Art. Hypertonus } \\
\hline Sturz & 378 & 78,6 & $1,1(0,81-1,5)$ & 0,54 \\
\hline Kein Sturz & 370 & 76,9 & & \\
\hline \multicolumn{5}{|c|}{ Laborwerte bei Aufnahme } \\
\hline \multicolumn{5}{|l|}{ Anämie } \\
\hline Sturz & 329 & 68,4 & $1,33(1,02-1,73)$ & 0,04 \\
\hline kein Sturz & 298 & 62,0 & & \\
\hline \multicolumn{5}{|c|}{ Hyponatriämie } \\
\hline Sturz & 200 & 41,6 & $1,49(1,15-1,95)$ & 0,003 \\
\hline Kein Sturz & 155 & 32,2 & & \\
\hline \multicolumn{5}{|c|}{ Hypernatriämie } \\
\hline Sturz & 9 & 1,9 & $1,13(0,43-2,95)$ & 0,81 \\
\hline Kein Sturz & 8 & 1,7 & & \\
\hline \multicolumn{5}{|c|}{ Hypokaliämie } \\
\hline Sturz & 64 & 13,3 & $0,96(0,67-1,4)$ & 0,85 \\
\hline Kein Sturz & 66 & 13,7 & & \\
\hline \multicolumn{5}{|c|}{ Hyperkaliämie } \\
\hline Sturz & 9 & 1,9 & $2,27(0,7-7,43)$ & 0,28 \\
\hline Kein Sturz & 5 & 1,0 & & \\
\hline \multicolumn{5}{|c|}{ Hypokalziämie } \\
\hline Sturz & 160 & 33,3 & $1,01(0,78-1,33)$ & 0,89 \\
\hline Kein Sturz & 158 & 32,9 & & \\
\hline \multicolumn{5}{|c|}{ Hyperkalziämie } \\
\hline Sturz & 9 & 1,9 & $1,51(0,53-4,27)$ & 0,43 \\
\hline Kein Sturz & 6 & 1,3 & & \\
\hline
\end{tabular}




\begin{tabular}{|l|l|l|l|l|}
\hline \multicolumn{6}{|l|}{ Kreatinin erhöht } \\
\hline Sturz & 279 & 58,0 & $1,09(0,85-1,42)$ & 0,47 \\
\hline Kein Sturz & 268 & 55,7 & & \\
\hline
\end{tabular}

\begin{tabular}{|c|c|c|c|c|}
\hline \multicolumn{5}{|c|}{ Leukozytopenie } \\
\hline Sturz & 18 & 3,7 & $1,83(0,84-4,01)$ & 0,12 \\
\hline Kein Sturz & 10 & 2,1 & & \\
\hline \multicolumn{5}{|c|}{ Leukozytose } \\
\hline Sturz & 188 & 39,1 & $1,40(1,08-1,83)$ & 0,01 \\
\hline Kein Sturz & 151 & 31,4 & & \\
\hline \multicolumn{5}{|c|}{$C R P>5 \mathrm{mg} / \mathrm{l}$} \\
\hline Sturz & 153 & 31,8 & $1,29(0,91-1,84)$ & 0,02 \\
\hline Kein Sturz & 120 & 24,9 & & \\
\hline \multicolumn{5}{|c|}{ Vitalparameter am Sturztag/ Tag. 5 nach Aufnahme in der Kontrollgruppe } \\
\hline \multicolumn{5}{|c|}{ Blutdruck $>140 / 90$ * } \\
\hline Sturz & 14 & 3,2 & $3,63(1,18-11,15)$ & 0,02 \\
\hline Kein Sturz & 4 & 0,9 & & \\
\hline \multicolumn{5}{|c|}{ Blutdruck <90/70 } \\
\hline Sturz & 2 & 0,4 & $0,5(0,04-5,6)$ & 1 \\
\hline Kein Sturz & 1 & 0,2 & & \\
\hline \multicolumn{5}{|c|}{ Temperatur $>37,5$ * } \\
\hline Sturz & 12 & 3,1 & $1,09(0,46-2,54)$ & 0,84 \\
\hline Kein Sturz & 10 & 2,8 & & \\
\hline \multicolumn{5}{|c|}{ Herzfrequenz $>100$ * } \\
\hline Sturz & 18 & 4,3 & $1,56(0,74-3,28)$ & 0,24 \\
\hline Kein Sturz & 12 & 2,8 & & \\
\hline \multicolumn{5}{|c|}{ Herzfrequenz $<60$} \\
\hline Sturz & 18 & 4,3 & $1,23(0,62-2,49)$ & 0,54 \\
\hline Kein Sturz & 15 & 3,5 & & \\
\hline
\end{tabular}

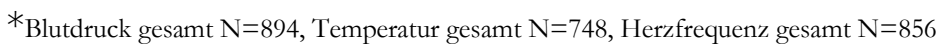

Tabelle 36 Hauptdiagnosen nach ICD-10 2013

\begin{tabular}{|l|r|r|r|r|}
\hline \multicolumn{1}{|l|}{ Hauptdiagnosen ICD-10 2013} & N & $\%$ & \multicolumn{2}{|c|}{ univariate OR (95KI) } \\
\hline A00-B99 Bestimmte infektiöse und parasitäre Krankheiten & \\
\hline Sturz & 20 & 4,2 & $1,26(0,65-2,46)$ & 0,49 \\
\hline Kein Sturz & 16 & 3,3 & & \\
\hline
\end{tabular}


C00-D48 Neubildungen

Sturz

Kein Sturz

\begin{tabular}{|l|l|l}
\hline 53 & \\
\hline 3 &
\end{tabular}

11,0

6,9

D50-D90 Krankheiten des Blutes und der blutbildenden Organe sowie bestimmte Störungen mit Beteiligung des Immunsystems

\begin{tabular}{|l|r|r|r|r|}
\hline Sturz & 6 & 1,3 & $1,20(0,36-3,97)$ & 0,76 \\
\hline Kein Sturz & 5 & 1,0 & & \\
\hline
\end{tabular}

E00-E90 Endokrine, Ernährungs- und Stoffwechselkrankheiten

\begin{tabular}{|l|r|r|r|r|}
\hline Sturz & 23 & 4,8 & $1,56(0,63-2,14)$ & 0,64 \\
\hline Kein Sturz & 20 & 4,2 & & \\
\hline
\end{tabular}

\section{F00-F99 Psychische und Verhaltensstörungen}

Sturz

Kein Sturz

\begin{tabular}{|l|l|}
\hline 5 & \\
5 &
\end{tabular}

1,0

1,0

$1(0,28-3,48)$

G00-G99 Krankheiten des Nervensystems

\begin{tabular}{|l|r|r|r|r|}
\hline Sturz & 16 & 3,3 & $1,15(0,55-2,38)$ & 0,71 \\
\hline Kein Sturz & 14 & 2,9 & & \\
\hline
\end{tabular}

H00-H59 Krankheiten des Auges und der Augenanhangsgebilde

\begin{tabular}{|l|r|r|r|r}
\hline Sturz & 0 & 0 & 0 & 0 \\
\hline Kein Sturz & 0 & 0 & & \\
\hline
\end{tabular}

H60-H95 Krankheiten des Ohres und des Warzenfortsatzes

\begin{tabular}{|l|r|r|r|r|}
\hline Sturz & 0 & 0 & 0 & 0 \\
\hline Kein Sturz & 0 & 0 & & \\
\hline
\end{tabular}

100-199 Krankheiten des Kreislaufsystems

\begin{tabular}{|l|r|r|r|r|}
\hline Sturz & 94 & 19,5 & $1,15(0,83-1,59)$ & 0,41 \\
\hline Kein Sturz & 84 & 17,8 & & \\
\hline
\end{tabular}

\section{J00-J99 Krankheiten des Atmungssystems}

\begin{tabular}{|c|c|c|c|c|}
\hline Sturz & 34 & 7,1 & $0,49(0,32-0,76)$ & 0,001 \\
\hline Kein Sturz & 64 & 13,1 & & \\
\hline \multicolumn{5}{|c|}{ K00-K93 Krankheiten des Verdauungssystems } \\
\hline Sturz & 32 & 6,6 & $0,54(0,34-0,85)$ & 0,01 \\
\hline Kein Sturz & 56 & 11,6 & & \\
\hline \multicolumn{5}{|c|}{ L00-L99 Krankheiten der Haut und der Unterhaut } \\
\hline Sturz & 3 & 0,6 & $3,10(0,31-29,04)$ & 0,62 \\
\hline Kein Sturz & 1 & 0,2 & & \\
\hline
\end{tabular}

M00-M99 Krankheiten des Muskel-Skelett-Systems und des Bindegewebes

\begin{tabular}{|l|r|r|r|r|}
\hline Sturz & 38 & 7,9 & $0,69(0,45-1,07)$ & 0,1 \\
\hline Kein Sturz & 53 & 11,0 & & \\
\hline N00-N99 Krankheiten des Urogenitalsystems \\
\hline
\end{tabular}




\begin{tabular}{|l|c|r|r|r|}
\hline Sturz & 50 & 10,4 & $1,48(0,94-2,32)$ & 0,09 \\
\hline Kein Sturz & 35 & 7,3 & & \\
\hline
\end{tabular}

\begin{tabular}{|c|c|c|c|c|}
\hline \multicolumn{5}{|c|}{ 000-099 Schwangerschaft, Geburt und Wochenbett } \\
\hline Sturz & 0 & 0 & 0 & 1 \\
\hline Kein Sturz & 0 & 0 & & \\
\hline \multicolumn{5}{|c|}{ P00-P96 Bestimmte Zustände, die ihren Ursprung in der Perinatalperiode haben } \\
\hline Sturz & 0 & 0 & 0 & 1 \\
\hline Kein Sturz & 0 & 0 & & \\
\hline \multicolumn{5}{|c|}{ Q00-Q99 Angeborene Fehlbildungen, Deformitäten und Chromosomenanomalien } \\
\hline Sturz & 0 & 0 & 0 & 1 \\
\hline Kein Sturz & 0 & 0 & & \\
\hline \multicolumn{5}{|c|}{ R00-R99 Symptome und abnorme klinische und Laborbefunde, die anderenorts nicht klassifiziert sind } \\
\hline Sturz & 10 & 2,1 & $0,9(0,38-2,16)$ & 0,83 \\
\hline Kein Sturz & 11 & 2,3 & & \\
\hline \multicolumn{5}{|c|}{ s00-T98 Verletzungen, Vergiftungen und bestimmte andere Folgen äußerer Ursachen } \\
\hline Sturz & 97 & 20,2 & $1,24(0,90-1,73)$ & 0,18 \\
\hline Kein Sturz & 81 & 16,8 & & \\
\hline \multicolumn{5}{|c|}{ U00-U99 Schlüsselnummern für besondere Zwecke } \\
\hline Sturz & 0 & 0 & & \\
\hline Kein Sturz & 1 & 0,2 & & \\
\hline \multicolumn{5}{|c|}{ v01-Y84 Äußere Ursachen von Morbidität und Mortalität } \\
\hline Sturz & 0 & 0 & 0 & 1 \\
\hline Kein Sturz & 0 & 0 & & \\
\hline \multicolumn{5}{|c|}{$\begin{array}{l}\text { Z00-Z99 Faktoren, die den Gesundheitszustand beeinflussen und zur Inanspruchnahme des Gesundheits- } \\
\text { wesens führen }\end{array}$} \\
\hline Sturz & 0 & 0 & & \\
\hline Kein Sturz & 1 & 0,2 & & \\
\hline
\end{tabular}

Tabelle 37 Einflussvariablen im Sturzjahr 2014

\begin{tabular}{|c|c|c|}
\hline Medikamente & $\mathrm{N}$ & $\%$ \\
\hline \multicolumn{3}{|c|}{ Benzodiazepine kurz- und mittellang wirksam } \\
\hline Sturz & 78 & 16,2 \\
\hline \multicolumn{3}{|l|}{ Benzodiazepine lang wirksam } \\
\hline Sturz & 16 & 3,3 \\
\hline
\end{tabular}




\section{Neuroleptika antipsychotisch}

\begin{tabular}{|l|r|r|}
\hline Sturz & 40 & 8,3 \\
\hline
\end{tabular}

Neuroleptika sedierend

Sturz

\section{Neuroleptika andere}

Sturz

SNRI

Sturz

SSRI

Sturz

TZA

Sturz

23

Tetrazyklische Antidepressiva

Sturz

\section{Z-Substanz}

Sturz

andere Sedativa

Sturz

Antikonvulsiva

Sturz

\section{ACE-Hemmer/Sartane}

Sturz

\section{B-Blocker}

Sturz

\section{Kalzium-Antagonisten}

Sturz

Aldosteronrezeptor-Antagonisten

Sturz

Alpha-2-Agonisten/ Alpha-1-Antagonisten

Sturz

\section{Antihypertensiva nach PRISCUS-Liste nicht empfohlen}

Sturz

Antiarrhythmika nach PRISCUS-Liste empfohlen

Sturz

\section{Antiarrhythmika nach PRISCUS-Liste nicht empfohlen}

Sturz

\section{Opiate}

Sturz 
Nebendiagnosen

Parkinsonsyndrom

Sturz

\section{Diabetes mellitus}

\begin{tabular}{|l|r|r|}
\hline Sturz & 119 & 24,7 \\
\hline KHK & 139 & 28,9 \\
\hline Sturz & \multicolumn{3}{|c|}{} \\
\hline
\end{tabular}

\section{Herzinsuffizienz}

Sturz

Demenz

Sturz

\section{Ischämischer Schlaganfall}

Sturz

Epilepsie

Sturz

Exsikkose

Sturz

Delir

Sturz

Art. Hypertonus

Sturz

Laborwerte bei Aufnahme

\section{Anämie}

Sturz

\section{Hyponatriämie}

Sturz

Hypernatriämie

Sturz

Hypokaliämie

Sturz

\section{Hyperkaliämie}

Sturz

\section{Hypokalziämie}

Sturz

Hyperkalziämie

Sturz 


\begin{tabular}{|l|r|r|}
\hline Sturz & 288 & 60,0 \\
\hline Leukozytopenie & 19 & 4,0 \\
\hline Sturz & 19 & \multicolumn{3}{|c|}{} \\
\hline
\end{tabular}

\begin{tabular}{|c|c|c|}
\hline \multicolumn{3}{|c|}{ Leukozytose } \\
\hline Sturz & 181 & 37,6 \\
\hline \multicolumn{3}{|c|}{$C R P>5 \mathrm{mg} / \mathrm{l}$} \\
\hline Sturz & 137 & 28,5 \\
\hline \multicolumn{3}{|c|}{ Vitalparameter Tag des Sturzes } \\
\hline \multicolumn{3}{|c|}{ Blutdruck > 140/90* } \\
\hline Sturz & 7 & 1,5 \\
\hline
\end{tabular}

Blutdruck <90/60

Sturz

8

Temperatur $>37,5$ *

Sturz

Herzfrequenz $>100$ *

Sturz

9

Herzfrequenz $<60$

Sturz

BMI

BMI 25-30 *

Sturz

BMI $>30$

80

BMI $>30$

Sturz

50

$B M I<17,5$

Sturz

Geriatrische Assessments

Timed-Up-and Go schnell *

Sturz

Timed-Up-and Go intermediate

Sturz

4

Timed-Up-and Go langsam

Sturz

Barthel-Index pflegebedürftig *

Sturz

Barthel-Index hilfsbedürftig

Sturz 


\begin{tabular}{|l|r|r|}
\hline Sturz & 8 & 4,4 \\
\hline Tinetti-Test <20 Punkte * & \multicolumn{3}{|c|}{} \\
\hline Sturz & 127 & 91,4 \\
\hline
\end{tabular}

\begin{tabular}{|c|c|c|}
\hline \multicolumn{3}{|c|}{ MMST- schwere Demenz * } \\
\hline Sturz & 4 & 4,9 \\
\hline \multicolumn{3}{|c|}{ MMST- moderate Demenz } \\
\hline Sturz & 23 & 28,0 \\
\hline \multicolumn{3}{|c|}{ MMST- leichte Demenz } \\
\hline Sturz & 29 & 35,3 \\
\hline \multicolumn{3}{|c|}{ MMST- keine Demenz } \\
\hline Sturz & 18 & 21,9 \\
\hline \multicolumn{3}{|c|}{$P S R$ * } \\
\hline Sturz & 27 & 21,8 \\
\hline \multicolumn{3}{|c|}{$A S R$ * } \\
\hline Sturz & 59 & 59,0 \\
\hline \multicolumn{3}{|c|}{ OP vor 4 Wochen untere Extremität } \\
\hline Sturz & 38 & 7,9 \\
\hline \multicolumn{3}{|c|}{ OP vor 4 Wochen obere Extremität } \\
\hline Sturz & 13 & 2,7 \\
\hline \multicolumn{3}{|c|}{ Kombinationen verschiedener Psychoaktiver Medikamente } \\
\hline \multicolumn{3}{|c|}{ Benzodiazepin kurz- und mittellang und lang-wirksam } \\
\hline Sturz & 4 & 0,8 \\
\hline \multicolumn{3}{|c|}{ Benzodiazepin und tetrazyklisches Antidepressivum } \\
\hline Sturz & 27 & 5,6 \\
\hline \multicolumn{3}{|c|}{ Benzodiazepin und Z-Substanz } \\
\hline Sturz & 4 & 0,8 \\
\hline \multicolumn{3}{|c|}{ tetrazyklisches Antidepressivum und Z-Substanz } \\
\hline Sturz & 17 & 3,5 \\
\hline \multicolumn{3}{|c|}{ tetrazyklisches Antidepressivum und sedierendes Neuroleptikum } \\
\hline Sturz & 15 & 3,1 \\
\hline \multicolumn{3}{|c|}{ Z-Substanz und sedierendes Neuroleptikum } \\
\hline Sturz & 2 & 0,4 \\
\hline \multicolumn{3}{|c|}{ Benzodiazepin und sedierendes Neuroleptikum } \\
\hline Sturz & 18 & 3,7 \\
\hline \multicolumn{3}{|c|}{ SSRI und Z-Substanz } \\
\hline Sturz & 3 & 0,6 \\
\hline
\end{tabular}




\begin{tabular}{|l|r|r|}
\hline Sturz & 11 & 2,3 \\
\hline SSRI und tetrazyklisches Antidepressivum & 7 & 1,5 \\
\hline Sturz & 7 & \multicolumn{2}{|c|}{} \\
\hline
\end{tabular}

\begin{tabular}{|c|c|c|}
\hline \multicolumn{3}{|c|}{ SSRI und sedierendes Neuroleptikum } \\
\hline Sturz & 2 & 0,4 \\
\hline \multicolumn{3}{|c|}{ SNRI und Z-Substanz } \\
\hline Sturz & 4 & 0,8 \\
\hline \multicolumn{3}{|c|}{ SNRI und Benzodiazepin } \\
\hline Sturz & 8 & 1,7 \\
\hline \multicolumn{3}{|c|}{ SNRI und tetrazyklisches Antidepressivum } \\
\hline Sturz & 13 & 2,7 \\
\hline \multicolumn{3}{|c|}{ SNRI und sedierendes Neuroleptikum } \\
\hline Sturz & 2 & 0,4 \\
\hline
\end{tabular}

*BD gesamt: N=459; Temperatur gesamt: $\mathrm{N}=406$; HF gesamt $\mathrm{N}=439$; BMI gesamt N=264; Timed-Up-and-Go-Test gesamt $\mathrm{N}=43$; Barthel-Index gesamt $\mathrm{N}=182$; Tinetti-Test gesamt $\mathrm{N}=139$; MMST gesamt $\mathrm{N}=82$; PSR gesamt $\mathrm{N}=124$; ASR gesamt $\mathrm{N}=100$

Tabelle 38 Hauptdiagnosen nach ICD-10 im Sturzjahr 2014

\begin{tabular}{|c|c|c|}
\hline Hauptdiagnosen ICD-10 2014 & $\mathbf{N}$ & $\%$ \\
\hline \multicolumn{3}{|c|}{ A00-B99 Bestimmte infektiöse und parasitäre Krankheiten } \\
\hline Sturz & 21 & 4,4 \\
\hline \multicolumn{3}{|l|}{ C00-D48 Neubildungen } \\
\hline Sturz & 35 & 7,3 \\
\hline \multicolumn{3}{|c|}{$\begin{array}{l}\text { D50-D90 Krankheiten des Blutes und der blutbildenden Organe sowie bestimmte Störungen mit Beteili- } \\
\text { gung des Immunsystems }\end{array}$} \\
\hline Sturz & 5 & 1,0 \\
\hline \multicolumn{3}{|c|}{ E00-E90 Endokrine, Ernährungs- und Stoffwechselkrankheiten } \\
\hline Sturz & 20 & 4,2 \\
\hline \multicolumn{3}{|c|}{ F00-F99 Psychische und Verhaltensstörungen } \\
\hline Sturz & 8 & 1,7 \\
\hline \multicolumn{3}{|l|}{ G00-G99 Krankheiten des Nervensystems } \\
\hline Sturz & 10 & 2,1 \\
\hline \multicolumn{3}{|c|}{ H00-H59 Krankheiten des Auges und der Augenanhangsgebilde } \\
\hline Sturz & 0 & 0 \\
\hline \multicolumn{3}{|c|}{ H60-H95 Krankheiten des Ohres und des Warzenfortsatzes } \\
\hline Sturz & 0 & 0 \\
\hline
\end{tabular}




\begin{tabular}{|c|c|c|}
\hline Sturz & 86 & 17,9 \\
\hline \multicolumn{3}{|c|}{ J00-J99 Krankheiten des Atmungssystems } \\
\hline Sturz & 49 & 10,2 \\
\hline \multicolumn{3}{|c|}{ K00-K93 Krankheiten des Verdauungssystems } \\
\hline Sturz & 36 & 7,5 \\
\hline \multicolumn{3}{|c|}{ L00-L99 Krankheiten der Haut und der Unterhaut } \\
\hline Sturz & 4 & 0,8 \\
\hline \multicolumn{3}{|c|}{ M00-M99 Krankheiten des Muskel-Skelett-Systems und des Bindegewebes } \\
\hline Sturz & 37 & 7,7 \\
\hline \multicolumn{3}{|c|}{ N00-N99 Krankheiten des Urogenitalsystems } \\
\hline Sturz & 49 & 10,2 \\
\hline \multicolumn{3}{|c|}{ 000-099 Schwangerschaft, Geburt und Wochenbett } \\
\hline Sturz & 0 & 0 \\
\hline \multicolumn{3}{|c|}{ P00-P96 Bestimmte Zustände, die ihren Ursprung in der Perinatalperiode haben } \\
\hline Sturz & 0 & 0 \\
\hline \multicolumn{3}{|c|}{ Q00-Q99 Angeborene Fehlbildungen, Deformitäten und Chromosomenanomalien } \\
\hline Sturz & 0 & 0 \\
\hline \multicolumn{3}{|c|}{ R00-R99 Symptome und abnorme klinische und Laborbefunde, die anderenorts nicht klassifiziert sind } \\
\hline Sturz & 12 & 2,5 \\
\hline \multicolumn{3}{|c|}{ S00-T98 Verletzungen, Vergiftungen und bestimmte andere Folgen äußerer Ursachen } \\
\hline Sturz & 108 & 22,5 \\
\hline \multicolumn{3}{|c|}{ U00-U99 Schlüsselnummern für besondere Zwecke } \\
\hline Sturz & 0 & 0 \\
\hline \multicolumn{3}{|c|}{ V01-Y84 Äußere Ursachen von Morbidität und Mortalität } \\
\hline Sturz & 0 & 0 \\
\hline \multicolumn{3}{|c|}{$\begin{array}{l}\text { Z00-Z99 Faktoren, die den Gesundheitszustand beeinflussen und zur Inanspruchnahme des Gesundheits- } \\
\text { wesens führen }\end{array}$} \\
\hline Sturz & 0 & 0 \\
\hline
\end{tabular}


Tabelle 39 Opioid-Umrechnungstabelle

\begin{tabular}{|c|c|c|c|c|c|c|c|c|c|c|c|c|c|}
\hline Substanz & \multicolumn{11}{|c|}{ Dosierung in $\mathrm{mg}$} & \multicolumn{2}{|c|}{ Faktor } \\
\hline Tramadol (oral / rectal) & 150 & 300 & 450 & 600 & & & & & & & & 5 & \\
\hline Tilidin / Naloxon (oral) & 150 & 300 & 450 & 600 & & & & & & & & 5 & \\
\hline Dihydrocodein (oral) & 120 & 240 & 360 & & & & & & & & & 4 & \\
\hline MORPHIN (oral / rectal) & 30 & 60 & 90 & 120 & 150 & 180 & 210 & 240 & 300 & 600 & 900 & 1 & 3 \\
\hline Oxycodon (oral) & 20 & 40 & 60 & 80 & 100 & 120 & 140 & 160 & 200 & & & 0,65 & \\
\hline L-Methadon (oral) & 7,5 & \multicolumn{10}{|c|}{ individuelle Titration } & 0,25 & 0,75 \\
\hline Hydromorphon (oral) & 4 & 8 & 12 & 16 & 20 & 24 & 28 & 32 & 40 & 80 & 120 & 0,13 & \\
\hline Buprenorphin (s.I.) & 0,4 & 0,8 & 1,2 & 1,6 & 2,0 & 2,4 & 2,8 & 3,2 & 3,2 & 3,6 & 4,0 & 0,01 & \\
\hline Tramadol (s.c. / i.m. / i.v.) & 100 & 200 & 300 & 400 & 500 & & & & & & & & 10 \\
\hline MORPHIN (s.c. / i.m. / i.v.) & 10 & 20 & 30 & 40 & 50 & 60 & 70 & 80 & 100 & 200 & 300 & 0,33 & 1 \\
\hline Hydromorphon (i.v.) & 2 & 4 & 6 & 8 & & & & & & & & & 0,5 \\
\hline Oxycodon (s.c. / i.v.) & 7,5 & 15 & 22,5 & 30 & & & & & & & & & 7,5 \\
\hline Piritramid (i.v.) & 15 & 30 & 45 & 60 & & & & & & & & & 1,5 \\
\hline Pethidin (i.v.) & 75 & 150 & 225 & 300 & & & & & & & & & 7,5 \\
\hline Buprenorphin (i.v. / i.m. / i.v.) & 0,3 & 0,6 & 0,9 & 1,2 & 1,5 & 1,8 & 2,1 & 2,4 & 3,0 & & & & 0,03 \\
\hline Morphin (epidural) & 2,5 & 5,0 & 7,5 & $\overline{10,0}$ & 12,5 & 15,0 & 17,5 & 20,0 & 25,0 & 50,0 & 75,0 & 0,08 & 0,25 \\
\hline Morphin (intrathekal) & 0,25 & 0,5 & 0,75 & 1,0 & 1,25 & 1,5 & 1,75 & 2,0 & 2,5 & 5,0 & 7,5 & 0,01 & 0,03 \\
\hline Buprenorphin TDS $(\mu \mathrm{g} / \mathrm{h})$ & \multicolumn{2}{|c|}{35} & 52,6 & 70 & 87,5 & 105 & 120 & 140 & & & & & \\
\hline Fentanyl TTS (mg/24h) & - & 0,6 & - & 1,2 & - & 1,8 & - & 2,4 & 3,0 & - & 9,0 & 0,01 & 0,03 \\
\hline Fentanyl TTS $(\mu \mathrm{g} / \mathrm{h})$ & - & 25 & - & 50 & - & 75 & - & 100 & 125 & - & 375 & 0,4 & 1,25 \\
\hline
\end{tabular}




\section{amedes \\ integrated diagnostics

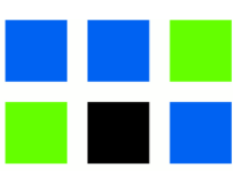

$\underline{\text { Referenzwerte Labor Evangelisches Krankenhaus-Weende }}$

Natrium:

$136-145 \mathrm{mmol} / \mathrm{L}$

Kalium:

$3,5-4,5 \mathrm{mmol} / \mathrm{L}$

Kalzium:

$2,15-2,55 \mathrm{mmol} / \mathrm{L}$

C-reaktives Protein:

$0-0,5 \mathrm{mg} / \mathrm{dL}$

Kreatinin:

$0,67-1,17 \mathrm{mg} / \mathrm{dL}$

Leukozyten:

3,98-10,04 G/L

Hämoglobin:

Frauen: $11,2-15,7 \mathrm{~g} / \mathrm{dL}$

Männer: 13,7-17,5 g/dL 


\section{Evangelisches Krankenhaus Göttingen-Weende gGmbH}

\section{Akademisches Lehrkrankenhaus der Georg-August-Universität Göttingen}

\section{Sturzprotokoll}

\section{Fallnummer: \\ Patient: \\ Geb.-Datum:}

\section{Pflegekraft:}

\begin{tabular}{|ll|ll|}
\hline \multicolumn{4}{|c|}{ Ursachen zum Sturz } \\
\hline Schwindel & $\square$ & Kraftlosigkeit & $\square$ \\
\hline gestolpert & $\square$ & Fremdeinwirkung & $\square$ \\
\hline ausgerutscht & $\square$ & keine Erinnerung & \} $\\
{\hline \text { Gangunsicherheit }} &{\square} &{ } &{ } \\
{\hline}$
\end{tabular}

\begin{tabular}{|l|c|}
\hline \multicolumn{2}{|c|}{ Ort? } \\
\hline Patienten-Zimmer & $\nabla$ \\
\hline Waschraum im Pat.-Zimmer & $\square$ \\
\hline Stationsflur & $\square$ \\
\hline Tagesraum & $\square$ \\
\hline Außerhalb des Gebäudes & $\square$ \\
\hline Andere Orte (z.B. Therapie) & $\square$ \\
\hline
\end{tabular}

\begin{tabular}{|lr|r|}
\hline Bettgitter angeordnet & ja $\square$ & nein $\square$ \\
\hline Bettgitter & oben $\square$ & unten $\square$ \\
\hline
\end{tabular}

\begin{tabular}{|ll|ll|}
\hline Sturzfolgen & & Lokalisation & \\
\hline Keine Folgen & $\square$ & Kopf & $\square$ \\
\hline Platzwunde & $\square$ & Stirn & $\square$ \\
\hline Schürfwunde & $\square$ & Schulter & $\square$ \\
\hline Prellmarke & $\square$ & Rücken & $\square$ \\
\hline Schmerz & $\square$ & Arm & $\square$ \\
\hline Fraktur & $\square$ & Hüfte & $\square$ \\
\hline & & Bein & $\square$ \\
\cline { 2 - 3 } & Thorax vorne & $\square$ \\
\cline { 2 - 3 } & Thorax seittich & $\square$ \\
\cline { 2 - 3 } & & & \\
\end{tabular}

\section{Station:}

Datum: 07.04.13
Uhrzeit: 11:10

\begin{tabular}{|l|c|}
\hline \multicolumn{2}{|c|}{ Hilfsmittel? } \\
\hline Keine Hilfsmittel & $\square$ \\
\hline Handstock / Unterarmstützen & $\square$ \\
\hline Hoher Gehwagen & $\square$ \\
\hline Vierrädrige Gehhilfe & $\square$ \\
\hline Trochanterschutz & $\square$ \\
\hline Wechseldruckmatratze & $\square$ \\
\hline Kein festes Schuhwerk & $\square$ \\
\hline Gehbock fest & $\square$ \\
\hline Gehbock flexibel & $\square$ \\
\hline
\end{tabular}

Pat. auf den Knien hockend vorm Tisch vorgefunden. Sie kann sich nicht erinnern, warum sie zu Boden geglitten/gefallen ist. Gibt Schmerzen in beiden Knien. Kann aber ohne Probleme stehen und laufen. Pat. ist adäquat.

\begin{tabular}{|l|l|ll|l|}
\hline \multicolumn{5}{|c|}{ Durchgeführte Maßnahmen } \\
\hline \multicolumn{2}{|c|}{ Erstmaßnahmen } & \multicolumn{3}{c|}{ nach Arztverordnung (AVO) } \\
\hline Lagerung nach Notwendigkeit & $\square$ & Röntgen & $\square$ & Infusionsgabe \\
\hline Blustillung $\square$ Verband \\
\hline RR und Pulsmessung & & CT: & $\square$ & Überleitung Intensivmedizin \\
\hline Blutzuckerkontrolle & $\square$ & Chirurgische Verrsorgung & $\square$ & \\
\hline
\end{tabular}

\begin{tabular}{|l|l|}
\hline Arztinformation & Arzt:
\end{tabular}




\section{$8 \quad$ Literaturverzeichnis}

Ackroyd-Stolarz S, Mackinnon NJ, Sketris I, Sabo B (2009): Potentially inappropriate prescribing of benzodiazepines for older adults and risk of falls during a hospital stay: a descriptive study. Can J Hosp Pharm $\underline{62}$ (4), 276-283

Alam A, Voronovich Z, Carley JA (2013): A review of therapeutic uses of mirtazapine in psychiatric and medical conditions. Prim Care Companion CNS Disord $\underline{15}$, pii: PCC.13r01525

Allan-Gibbs R (2010): Falls and hospitalized patients with cancer. A review of the literature. Clin J Oncol Nurs 14 (6), 784-792

Arnold I, Straube K, Himmel W, Heinemann S, Weiss V, Heyden L, Hummers-Pradier E, Nau R (2017): High prevalence of prescription of psychotropic drugs for older patients in a general hospital. BMC Pharmacol Toxicol 18, 76

Ashton H: Benzodiazepine abuse. In: Caan E, de Belleroche J (Hrsg.): Drink, drugs and dependence. Routledge, London 2002, 197-212

Ashton H (2005): The diagnosis and management of benzodiazepine dependence. Curr Opin Psychiatry 18 (3), 249-255

Babyak MA (2004): What you see may not be what you get: a brief, nontechnical introduction to overfitting in regression-type models. Psychosom Med $\underline{66}$ (3), 411-421

Bagley SC, White H, Golomb BA (2001): Logistic regression in the medical literature: standards for use and reporting, with particular attention to one medical domain. J Clin Epidemiol 4 (10), 979-985

Barkin RL (2007): Zolpidem extended-release. A single insomnia treatment option for sleep induction and sleep maintenance symptoms. Am J Ther 14 (3), 299-305

Barry E, Galvin R, Keogh C, Horgan F, Fahey T (2014): Is the Timed Up and Go test a useful predictor of risk of falls in community dwelling older adults. A systematic review and meta-analysis. BMC Geriatr 14, 14

Barry PJ, Gallagher P, Ryan C, O'mahony D (2007): START (screening tool to alert doctors to the right treatment)-an evidence-based screening tool to detect prescribing omissions in elderly patients. Age Ageing $\underline{36}$ (6), 632-638

Battegay E, Cheetham M, Holzer BM, Nowak A, Schmidt D, Rampini S (2017): Multimorbiditätsmanagement im Alltagsdilemma. Internist 58 (4), 344-353

Beauchet O, Fantino B, Allali G, Muir SW, Montero-Odasso M, Annweiler C (2011): Timed Up and Go test and risk of falls in older adults: a systematic review. J Nutr Health Aging 15 (10), 933-938 
Becker C, Rapp K (2011): Stürze in der Geriatrie. Diagnostik und Therapie. Internist $\underline{52}$ (8), 939-945

Benkert O, Regen F: Antidepressiva. In: Benkert O, Hippius H, Anghelescu I-G, Gründer G, Heiser P, Hiemke C, Himmerich H, Kiefer F, Lange-Asschenfeldt C, Müller MJ, et al. : Kompendium der Psychiatrischen Pharmakotherapie. 10. Auflage; Springer, Berlin Heidelberg 2014, 1-194

Benkert O, Steiger A: Medikamente zur Behandlung von Schlafstörungen. In: Benkert O, Hippius H, Anghelescu I-G, Gründer G, Heiser P, Hiemke C, Himmerich H, Kiefer F, Lange-Asschenfeldt C, Müller MJ, et al.: Kompendium der Psychiatrischen Pharmakotherapie. 10. Auflage; Springer, Berlin Heidelberg 2014, 512- 583

Berry SD, Miller RR (2008): Falls. Epidemiology, pathophysiology, and relationship to fracture. Curr Osteoporos Rep $\underline{6}$ (4), 149-154

Bischoff-Ferrari HA, Willett WC, Wong JB, Stuck AE, Staehelin HB, Orav EJ, Thoma A, Kiel DP, Henschkowski J (2009): Prevention of nonvertebral fractures with oral vitamin D and dose dependency: a meta-analysis of randomized controlled trials. Arch Intern Med $\underline{169}(6), 551-561$

Bischoff-Ferrari HA, Dawson-Hughes B, Orav EJ, Staehelin HB, Meyer, Otto W, Theiler R, Dick W, Willet WC, Egli A (2016): Monthly High-Dose Vitamin D Treatment for the Prevention of Functional Decline: A Randomized Clinical Trial. JAMA Intern Med 176 (2), 175-183

Blachman NL, Leipzig RM, Mazumdar M, Poeran J (2017): High-risk medications in hospitalized elderly adults. Are we making it easy to do the wrong thing? J Am Geriatr Soc $\underline{65}$ (3), 603-607

Böhm S: Pharmaka mit Wirkung auf das Nervensystem. In: Offermann S (Hrsg.): Pharmakologie und Toxikologie. Von den molekularen Grundlagen zur Pharmakotherapie. 2. Aufl. Berlin, Heidelberg 2016, 229-362

Bothwell LE, Greene JA, Podolsky SH, Jones DS (2016): Assessing the gold standardlessons from the history of RCTs. N Engl J Med $\underline{374}$ (22), 2175-2181

Bouillon K, Kivimaki M, Hamer M, Sabia S, Fransson EI, Singh-Manoux A, Gale CR, Batty GD (2013): Measures of frailty in population-based studies: an overview. BMC Geri$\operatorname{atr} \underline{13}, 64$

Bouldin EL, Andresen EM, Dunton NE, Simon M, Waters TM, Liu M, Daniels MJ, Mion LC, Shorr RI (2013): Falls among adult patients hospitalized in the United States: prevalence and trends. J Patient Saf $\underline{9}$ (1), 13-17

Boutin T, Kergoat MJ, Latour J, Massoud F, Kergoat H (2012): Vision in the global evaluation of older individuals hospitalized following a fall. J Am Med Dir Assoc 13 (2), 187.e159 
Boyd R, Stevens JA (2009): Falls and fear of falling. Burden, beliefs and behaviours. Age Ageing $\underline{38}$ (4), 423-428

Bueno-Cavanillas A, Padilla-Ruiz F, Jiménez-Moleón JJ, Peinado-Alonso CA, GálvezVargas R (2000): Risk factors in falls among the elderly according to extrinsic and intrinsic precipitating causes. Eur J Epidemiol 16 (9), 849-859

Bursac Z, Gauss CH, Williams DK, Hosmer DW (2008): Purposeful selection of variables in logistic regression. Source Code Biol Med 16 (12), 3-17

Cadenbach A: Tachyarrhythmien. In: Dietel M, Suttorp N, Zeitz M (Hrsg.): Harrisons Innere Medizin. Band 2., 18. Auflage; ABW Wissenschaftsverlag GmbH, Berlin 2012, 20152039

Campbell AJ, Robertson MC (2007): Rethinking individual and community fall prevention strategies: a meta-regression comparing single and multifactorial interventions. Age Ageing $\underline{36}(6), 656-662$

Carbonin P, Pahor M, Bernabei R, Sgadari A (1991): Is age an independent risk factor of adverse drug reactions in hospitalized medical patients? J Am Geriatr Soc $\underline{39}$ (11), 10931099

Carton L, Cottencin O, Lapeyre-Mestre M, Geoffroy PA, Favre J, Simon N, Nicolas S, Régis B, Benjamin R (2015): Off-Label Prescribing of Antipsychotics in Adults, Children and Elderly Individuals. A Systematic Review of Recent Prescription Trends. Curr Pharm Des 21 (23), 3280-3297

Cashin RP, Yang M (2011): Medications prescribed and occurrence of falls in general medicine inpatients. Can J Hosp Pharm $\underline{64}$ (5), 321-326

Chang CM, Chen MJ, Tsai CY, Ho LH, Hsieh HL, Chau YL, Liu CY (2011): Medical conditions and medications as risk factors of falls in the inpatient older people. A casecontrol study. Int J Geriatric Psychiatry 26 (6), 602-607

Chiu MH, Lee HD, Hwang HF, Wang SC, Lin MR (2015): Medication use and fall-risk assessment for falls in an acute care hospital. Geriatr Gerontol Int 15 (7), 856-863

Chow SK, Lai CK, Wong TK, Suen LK, Kong SK, Chan CK, Wong IY (2007): Evaluation of the Morse Fall Scale. Applicability in Chinese hospital populations. Int J Nurs Stud 44 (4), 556-565

Christenfeld NJ, Sloan RP, Carroll D, Greenland S (2004): Risk factors, confounding, and the illusion of statistical control. Psychosom Med $\underline{66}$ (6), 868-875

Cleophas TJ, Zwinderman AH (2007): Clinical trials: how to assess confounding and why so. Curr Clin Pharmacol 2 (2), 129-133

Concato J, Shah N, Horwitz RI (2000): Randomized, controlled trials, observational studies, and the hierarchy of research designs. N Engl J Med 342 (25), 1887-1892 
Cressie NA, Whitford HJ (1986): How to Use the Two Samplet-Test. Biom J 28 (2), 131148

Cumming RG, Le Couteur DG (2003): Benzodiazepines and risk of hip fractures in older people: a review of the evidence. CNS Drugs 17 (11), 825-837

Curcio CL, Gomez G, Reyes-Ortiz CA (2009): Activity restriction related to fear of falling among older people in the Colombian Andes mountains. Are functional or psychosocial risk factors more important? J Aging Health 21 (3), 460-479

Currie GM, Wheat JM, Kiat H (2011): Pharmacokinetic considerations for digoxin in older people. Open Cardiovasc Med J $\underline{5}, 130-135$

Darcourt G, Pringuey D, Sallière D, Lavoisy J (1999): The safety and tolerability of zolpidem--an update. J Psychopharmacol $\underline{13}$ (1), 81-93

Dennhardt R: Fieber und Hyperthermie. In: Dietel M, Suttorp N, Zeitz M (Hrsg.): Harrisons Innere Medizin. Band 1., 18. Auflage; ABW Wissenschaftsverlag GmbH, Berlin 2012, $156-160$

Divo MJ, Martinez CH, Mannino DM (2014): Ageing and the epidemiology of multimorbidity. Eur Respir J 44 (4), 1055-1068

Donald IP, Bulpitt CJ (1999): The prognosis of falls in elderly people living at home. Age Ageing 28 (2), 121-125

Dunlop BW, Davis PG (2008): Combination treatment with benzodiazepines and SSRIs for comorbid anxiety and depression. A review. Prim Care Companion J Clin Psychiatry 10 (3), 222-228

Dunne TJ, Gaboury I, Ashe MC (2014): Falls in hospital increase length of stay regardless of degree of harm. J Eval Clin Pract 20 (4), 396-400

Eckardt R, Steinhagen-Thiessen E, Kämpfe S, Buchmann N (2014): Polypharmazie und Arzneimitteltherapiesicherheit im Alter. Strategien zur Verbesserung. Z Gerontol Geriatr $\underline{47}$ (4), 293-301

ESC Guidelines for the diagnosis and treatment of acute and chronic heart failure. The Task Force for the diagnosis and treatment of acute and chronic heart failure of the European Society of Cardiology (ESC). Developed with the special contribution of the Heart Failure Association (HFA) of the ESC (2016). Eur Heart J 37 (27), 2129-2200

Evans D, Hodgkinson B, Lambert L, Wood J (2001): Falls risk factors in the hospital setting: a systematic review. Int J Nurs Pract $\underline{7}$ (1), 38-45

Evidence-based guideline for the management of high blood pressure in adults: report from the panel members appointed to the Eighth Joint National Committee (2014). JAMA $\underline{311}$ (5), 507-520 
Ferguson JM (2001): SSRI Antidepressant Medications. Adverse Effects and Tolerability. Prim Care Companion J Clin Psychiatry $\underline{3}$ (1), 22-27

Fernandez L, Cassagne-Pinel C (2001): Addiction aux benzodiazépines et symptomatologie anxieuse et dépressive chez les sujets âgés. Encephale 27 (5), 459-474

Ferrer A, Formiga F, Plana-Ripoll O, Tobella MA, Gil A, Pujol R (2012): Risk of falls in 85-year-olds is associated with functional and cognitive status: the Octabaix Study. Arch Gerontol Geriatr $\underline{54}$ (2), 352-356

Fischer ID, Krauss MJ, Dunagan WC, Birge S, Hitcho E, Johnson S, Costantinou E, Fraser VJ (2005): Patterns and predictors of inpatient falls and fall-related injuries in a large academic hospital. Infect Control Hosp Epidemiol 26 (10), 822-827

Foley DJ, Monjan AA, Brown SL, Simonsick EM, Wallace RB, Blazer DG (1995): Sleep complaints among elderly persons. An epidemiologic study of three communities. Sleep $\underline{18}$ (6), 425-432

Folstein MF, Folstein SE, McHugh PR (1975): "Mini-mental state". A practical method for grading the cognitive state of patients for the clinician. J Psychiatr Res 12 (3), 189-198 Fong TG, Tulebaev SR, Inouye SK (2009): Delirium in elderly adults: diagnosis, prevention and treatment. Nat Rev Neurol $\underline{5}$ (4), 210-220

Foy A., O'Connell D, Henry D, Kelly J, Cocking S, Halliday J (1995): Benzodiazepine use as a cause of cognitive impairment in elderly hospital inpatients. J Gerontol A Biol Sci Med Sci $\underline{50}(2), 99-106$

Frels C, Williams P, Narayanan S, Gariballa SE (2002): Iatrogenic causes of falls in hospitalised elderly patients: a case-control study. Postgrad Med J $\underline{78}$ (922), 487-489

Frerichmann U, Raschke MJ, Stöckle U, Wöhrmann S, Lohmann R (2007): Proximale Femurfrakturen im Alter. Krankenkassendaten von über 23 Mio. Versicherten--Teil 2. Unfallchirurg $\underline{110}$ (7), 610-616

Friedman SM, Munoz B, West SK, Rubin GS, Fried LP (2002): Falls and fear of falling. Which comes first? A longitudinal prediction model suggests strategies for primary and secondary prevention. J Am Geriatr Soc $\underline{50}$ (8), 1329-1335

Frohnhofen H, Michalek C, Wehling M (2011): Bewertung von Medikamenten in der Geriatrie mit der neuen FORTA-Klassifikation. Dtsch Med Wochenschr 136 (27), 1417-1421

Furukawa, TA, Streiner DL, Young LT (2001): Is antidepressant-benzodiazepine combination therapy clinically more useful? A meta-analytic study. J Affect Disord $\underline{65}$ (2), 173-177 Gales BJ, Menard SM (1995): Relationship between the administration of selected medications and falls in hospitalized elderly patients. Ann Pharmacother 29 (4), 354-358

Gangavati A, Hajjar I, Quach L, Jones RN, Kiely DK, Gagnon P, Lipsitz LA (2011): Hypertension, orthostatic hypotension, and the risk of falls in a community-dwelling elderly 
population. The maintenance of balance, independent living, intellect, and zest in the elderly of Boston study. J Am Geriatr Soc $\underline{59}$ (3), 383-389

Gankam Kengne F, Andres C, Sattar L, Melot C, Decaux G (2008): Mild hyponatremia and risk of fracture in the ambulatory elderly. QJM 101 (7), 583-588

Gates S, Smith La, Fisher JD, Lamb SE (2008): Systematic review of accuracy of screening instruments for predicting fall risk among independently living older adults. J Rehabil Res Dev $\underline{45}(8), 1105-1116$

Gavazzi G, Krause KH (2002): Ageing and infection. Lancet Infect Dis 2 (11), 659-666

Gogol M (2008): Das Delir im höheren Lebensalter. Z Gerontol Geriatr 41 (6), 431-439

Goodridge D, Marr H (2002): Factors associated with falls in an inpatient palliative care unit. An exploratory study. Int J Palliat Nurs $\underline{8}$ (11), 548-556

Graafmans WC, Ooms ME, Hofstee HM, Bezemer PD, Bouter LM, Lips P (1996): Falls in the elderly: a prospective study of risk factors and risk profiles. Am J Epidemiol $\underline{143}$ (11), 1129-1136

Graham J, Rockwood K, Beattie BL, Eastwood R, Gauthier S, Tuokko H, McDowell I (1997): Prevalence and severity of cognitive impairment with and without dementia in an elderly population. Lancet $\underline{349}$ (9068), 1793-1796

Greenland S: Introduction to regression models. In: Rothmann KJ, Greenland S (Hrsg.): Modern Epidemiology. 3. Auflage; Lippincott Williams \& Wilkins, Philadelphia 2008, 381 418

Gribbin J, Hubbard R, Gladman JR, Smith C, Lewis S (2010): Risk of falls associated with antihypertensive medication: population-based case-control study. Age Ageing $\underline{39}$ (5), 592597

Grimes DA, Schulz KF (2005): Compared to what? Finding controls for case-control studies. Lancet $\underline{365}$ (9468), 1429-1433

Guertin KA, Freedman ND, Neal D, Loftfield E, Graubard BI, Caporaso NE, Sinha R (2016): Coffee consumption and incidence of lung cancer in the NIH-AARP Diet and Health Study. Int J Epidemiol 45 (3), 929-939

Gulich M (2008): Sturzprävention bei älteren Menschen - eine Übersicht. Z Allg Med $\underline{84}$ (3), 116-119

Gunja N (2013): The clinical and forensic toxicology of Z-drugs. J Med Toxicol 9 (2), 155162

Hajak G, Müller WE, Wittchen HU, Pittrow D, Kirch W (2003): Abuse and dependence potential for the non-benzodiazepine hypnotics zolpidem and zopiclone. A review of case reports and epidemiological data. Addiction $\underline{98}$ (10), 1371-1378 
Ham AC, van Dijk SC, Swart KMA, Enneman AW, van der Zwaluw NL, BrouwerBrolsma EM, van Schoor NM, Zilikens MC; Lips P, De Groot LCPGM et al. (2017): Betablocker use and fall risk in older individuals: Original results from two studies with metaanalysis. Br J Clin Pharmacol $\underline{83}$ (10), 2292-2302

Hammond T, Wilson A (2013): Polypharmacy and falls in the elderly: a literature review. Nurs Midwifery Stud 2 (2), 171-175

Hansen J (1998): Common cancers in the elderly. Drugs Aging 13 (6), 467-478

Hartikainen S, Lonnroos E, Louhivuori K (2007): Medication as a risk factor for falls: critical systematic review. J Gerontol A Biol Sci Med Sci 62 (10), 1172-1181

Hauer K, Lamb SE, Jorstad EC, Todd C, Becker C (2006): Systematic review of definitions and methods of measuring falls in randomised controlled fall prevention trials. Age Ageing $\underline{35}(1), 5-10$

Heinemann S, Weiß V, Straube K, Nau R, Grimmsmann T, Himmel W, Hummers-Pradier E (2016): Understanding and reducing the prescription of hypnotics and sedatives at the interface of hospital care and general practice. A protocol for a mixed-methods study. BMJ Open $\underline{6}(8)$, e 011908

Heinrich S, Rapp K, Rissmann U, Becker C, König HH (2010): Cost of falls in old age: a systematic review. Osteoporos Int 21 (6), 891-902

Heinze C, Lahmann N, Dassen (2002): Sturzhäufigkeit in deutschen Kliniken. Gesundheitswesen $\underline{64}$ (11), 598-601

Hendrich AL, Ann L, Bender PS, Nyhuis A (2003): Validation of the Hendrich II Fall Risk Model: a large concurrent case/control study of hospitalized patients. Appl Nurs Res $\underline{16}$ (1), 9-21

Henschel F, Redaelli M, Siegel M, Stock S (2015): Correlation of Incident Potentially Inappropriate Medication Prescriptions and Hospitalization: An Analysis Based on the PRISCUS List. Drugs Real World Outcomes 2 (3), 249-259

Hepper HJ, Sieber C, Cornel S, Walger P, Bahrmann P, Singler K (2013): Infections in the elderly. Crit Care Clin 29 (3), 757-774

Herman T, Mirelman A, Giladi N, Schweiger A, Hausdorff, JM (2010): Executive control deficits as a prodrome to falls in healthy older adults. A prospective study linking thinking, walking, and falling. J Gerontol A Biol Sci Med Sci $\underline{65}$ (10), 1086-1092

Hignett S, Sands G, Griffiths P (2013): In-patient falls: what can we learn from incident reports? Age Ageing 42 (4), 527-531

Hill KD, Vu M, Walsh W (2007): Falls in the acute hospital setting--impact on resource utilisation. Aust Health Rev $\underline{31}$ (3), 471-477 
Hitcho EB, Krauss MJ, Birge S, Claiborne DW, Fischer I, Johnson S, Nast PA, Costatinou E, Fraser VJ (2004): Characteristics and circumstances of falls in a hospital setting: a prospective analysis. J Gen Intern Med 19 (7), 732-739

Holm KJ, Goa KL (2000): Zolpidem: an update of its pharmacology, therapeutic efficacy and tolerability in the treatment of insomnia. Drugs $\underline{59}$ (4), 865-889

Holt S, Schmiedl S, Thürmann PA (2010): Potentially inappropriate medications in the elderly: the PRISCUS list. Dtsch Arztebl Int 107 (31-32), 543-551

Homfeldt HG: Gesundheit und Krankheit im Alter. In: Kirsten A, Karl U (Hrsg.): Handbuch Soziale Arbeit und Alter. VS Verlag für Sozialwissenschaften, Wiesbaden 2010, 315320

Hosmer DW, Hosmer T, Le Cessie S, Lemeshow S (1997): A Comparison of Goodnessof-fit Tests for the Logistic Regression Model. Statist Med 16 (9), 965-980

Iaboni A, Flint AJ (2013): The complex interplay of depression and falls in older adults: a clinical review. Am J Geriatr Psychiatry 21 (5), 484-492

Icks A, Haastert B, Wildner M, Becker C, Meyer G (2008): Inzidenz von Hüftfrakturen in Deutschland - Auswertung der Krankenhausdiagnosestatistik 2004. Dtsch Med Wochenschr $\underline{133}$ (4), 125-128

Isaia G, Corsinovi L, Bo M, Santos-Pereira P, Michelis G, Aimonino N, Zanocchi M, (2011): Insomnia among hospitalized elderly patients. Prevalence, clinical characteristics and risk factors. Arch Gerontol Geriatr $\underline{52}$ (2), 133-137

Jansen S, Schoe J, van Rijn M, Abu-Hanna A, van Moll CEP, van der Velde N, Rooij, Sophia ED (2015): Factors associated with recognition and prioritization for falling, and the effect on fall incidence in community dwelling older adults. BMC Geriatr $\underline{15}, 169$ Joos AA (1998): Pharmakologische Interaktionen von Antibiotika und Psychopharmaka. Psychiatr Prax $\underline{25}$ (2), 57-60

Judd E, Calhoun DA (2012): Hypertension and orthostatic hypotension in older patients. J Hypertens $\underline{30}$ (1), 38-39

Kallin K, Gustafson Y, Sandman PO, Karlsson S (2004): Drugs and falls in older people in geriatric care settings. Aging Clin Exp Res $\underline{16}$ (4), 270-276

Katz IR, Ira R, Rupnow M, Kozma C, Schneider L (2004): Risperidone and Falls in Ambulatory Nursing Home Residents With Dementia and Psychosis or Agitation. Secondary Analysis of a Double-Blind, Placebo-Controlled Trial. Am J Geriatr Psychiatry 12 (5), 499_ 508

Kegelmeyer DA, Kloos AD, Thomas KM, Kostyk SK (2007): Reliability and validity of the Tinetti Mobility Test for individuals with Parkinson disease. Phys Ther $\underline{87}$ (10), 13691378 
Kellogg IWG (1987): The prevention of falls in later life. A report of the Kellogg International Work Group on the Prevention of Falls by the Elderly. Dan Med Bull 34 (4), 1-24

Kennedy WK, Jann MK, Kutscher EC (2013): Clinically significant drug interactions with atypical antipsychotics. CNS Drugs 27 (12), 1021-1048

Kinne V, Klewer J (2016): Sturzereignisse in einem Universitätsklinikum. HeilberufeScience $\underline{7}(1), 40-46$

Köpke S, Meyer G (2006): The Tinetti test. Babylon in geriatric assessment. Z Gerontol Geriatr $\underline{39}$ (4), 288-291

Krauss MJ, Evanoff B, Hitcho E, Ngugi KE, Dunagan WC, Fischer I, Birge S, Johnson S, Costantinou E, Fraser VJ (2005): A case-control study of patient, medication, and carerelated risk factors for inpatient falls. J Gen Intern Med $\underline{20}$ (2), 116-122

Krauss MJ, Nguyen SL, Dunagan WC, Birge S, Costantinou E, Johnson S, Caleca B, Fraser VJ (2007): Circumstances of patient falls and injuries in 9 hospitals in a midwestern healthcare system. Infect Control Hosp Epidemiol 28 (5), 544-550

Kuhn-Thiel AM, Weiß C, Wehling M (2014): Consensus validation of the FORTA (Fit fOR The Aged) List: a clinical tool for increasing the appropriateness of pharmacotherapy in the elderly. Drugs Aging $\underline{31}$ (2), 131-140

Kukreja S, Kalra G, Shah N, Shrivastava A (2013): Polypharmacy in psychiatry. A review. Mens Sana Monogr 11 (1), 82-99

Lahmann NA, Heinze C, Rommel A (2014): Stürze in deutschen Krankenhäusern und Pflegeheimen 2006-2013. Häufigkeiten, Verletzungen, Risikoeinschätzung und durchgeführte Prävention. Bundesgesundheitsblatt, Gesundheitsforschung, Gesundheitsschutz $\underline{57}$ (6), 650-659

Lamb SE, Jørstad-Stein EC, Hauer K, Becker C (2005): Development of a common outcome data set for fall injury prevention trials: the Prevention of Falls Network Europe consensus. J Am Geriatr Soc $\underline{53}$ (9), 1618-1622

Lamy PP (1988): Potential adverse effects of antihypertensive drugs in the elderly. J Hypertens $\underline{6}(1), 81-5$

Lane RM (1996): Pharmacokinetic drug interaction potential of selective serotonin reuptake inhibitors. Int Clin Psychopharmacol 11 (5), 31-61

Laroche ML, Charmes JP, Bouthier F, Merle L (2009): Inappropriate medications in the elderly. Clin Pharmacol Ther $\underline{85}$ (1), 94-97

Lavan A, Gallagher P (2016): Predicting risk of adverse drug reactions in older adults. Ther Adv Drug Saf $\underline{7}(1), 11-22$

Leipzig RM, Cumming RG, Tinetti ME (1999a): Drugs and falls in older people: a systematic review and meta-analysis: I. Psychotropic drugs. J Am Geriatr Soc $\underline{47}$ (1), 30-39 
Leipzig, RM, Cumming RG, Tinetti ME (1999b): Drugs and Falls in Older People: A Systematic Review and Meta-analysis: II. Cardiac and Analgesic Drugs. J Am Geriatr Soc 47 (1), 40-50

Leitlinie Bluthochdruck, s. Evidence-Based Guideline for the management of high blood pressure in adults: report from the panel members appointed to the Eighth Joint National Committee 2014

Leitlinie Herzinsuffizienz s. ESC Guidelines for the diagnosis and treatment of acute and chronic heart failure. The Task Force for the diagnosis and treatment of acute and chronic heart failure of the European Society of Cardiology (ESC). Developed with the special contribution of the Heart Failure Association (HFA) of the ESC 2016

Leitlinie Demenzen s. S3-Leitlinie Demenzen 2016

Leitlinie Multimedikation s. S2e-hausärztliche Leitlinie DEGAM 2014

Leitlinie Nicht erholsamer Schlaf/Schlafstörungen s. S3-Leitlinie Nicht erholsamer Schlaf/Schlafstörungen 2017

Letmaier M, Painold A, Holl AK, Vergin H, Engel R, Konstantinidis A, Kasper S, Grohmann R (2012): Hyponatraemia during psychopharmacological treatment: results of a drug surveillance programme. Int J Neuropsychopharmcol 15 (6), 739-748

Lim SC, Mamun K, Lim JKH (2014): Comparison between elderly inpatient fallers with and without dementia. Singapore Med J $\underline{55}$ (2), 67-71

Lipsitz LA, Gagnon M, Vyas M, Iloputaife I, Kiely DK, Sorond F, Serrador J, Cheng DM, Babikian V, Cupples LA (2005): Antihypertensive therapy increases cerebral blood flow and carotid distensibility in hypertensive elderly subjects. Hypertension $\underline{45}$ (2), 216221

Llorente MD, David D, Golden AG, Silverman MA (2000): Defining patterns of benzodiazepine use in older adults. J Geriatr Psychiatry Neurol 13 (3), 150-160

Lord SR, Tiedemann A, Chapman K, Munro B, Murray SM, Gerontology M, Ther GR, Sherrington $C$ (2005): The effect of an individualized fall prevention program on fall risk and falls in older people. A randomized, controlled trial. J Am Geriatr Soc $\underline{53}$ (8), 12961304

Lorenzl S, Fusgen I, Noachtar S (2012): Acute confusional States in the elderly--diagnosis and treatment. Dtsch Arztebl Int 109 (21), 391-400

Lübke N, Meinck M, von Renteln-Kruse W (2004): Der Barthel-Index in der Geriatrie. Eine Kontextanalyse zum Hamburger Einstufungsmanual. Z Gerontol Geriatr $\underline{37}$ (4), $316-326$ 
Lundin-Olsson L, Jensen J, Nyberg L, Gustafson Y (2003): Predicting falls in residential care by a risk assessment tool, staff judgement, and history of falls. Aging Clin Exp Res $\underline{15}$ (1), 51-59

MacKinnon D (2000): Equivalence of the Mediation, Confounding and Suppression Effect. Prevent Sci 1 (4), 173-181

Mahoney FI, Barthel DW (1965): Functional Evaluation: The Barthel Index. MD State Med J $\underline{14}, 61-65$

Maldonado G, Greenland S (1993): Simulation study of confounder-selection strategies. Am J Epidemiol 138 (11), 923-936

Mallet L, Spinewine A, Huang A (2007): The challenge of managing drug interactions in elderly people. Lancet $\underline{370}$ (9582), 185-191

Mancuso CE, Tanzi MG, Gabay M (2004): Paradoxical reactions to benzodiazepines. Literature review and treatment options. Pharmacother 24 (9), 1177-1185

Mandrioli R, Forti GC, Raggi MA (2006): Fluoxetine metabolism and pharmacological interactions. The role of cytochrome p450. Curr Drug Metab 7 (2), 127-133

Mangoni AA, Jackson SHD (2004): Age-related changes in pharmacokinetics and pharmacodynamics. Basic principles and practical applications. Br J Clin Pharmacol $\underline{57}$ (1), 6-14

Marengoni A, Angleman S, Melis R, Mangialasche F, Karp A, Garmen A, Meinow B, Fratiglioni L (2011): Aging with multimorbidity: a systematic review of the literature. Ageing Res Rev 10 (4), 430-439

Marik PE (2006): Management of the critically ill geriatric patient. Crit Care Med 34 (9), 176-182

Markun S, Holzer BM, Rodak R, Kaplan V, Wagner CC, Battegay E, Zimmerli L (2014): Therapeutic conflicts in emergency department patients with multimorbidity: a crosssectional study. PloS One $\underline{9}(10)$, e110309

Mazur K, Wilczyński K, Szewieczek J (2016): Geriatric falls in the context of a hospital fall prevention program: delirium, low body mass index, and other risk factors. Clin Interv Aging 11, 1253-1261

McMichael Ka, Vander Bilt J, Lavery L, Rodriguez E, Ganguli M (2008): Simple balance and mobility tests can assess falls risk when cognition is impaired. Geriatr Nurs $\underline{29}$ (5), $311-323$

Messiha FS (1993): Fluoxetine. Adverse effects and drug-drug interactions. J Clin Toxicol $\underline{31}(4), 603-630$

Michalek C, Wehling M, Schlitzer J, Frohnhofen H (2014): Effects of "Fit fOR The Aged" (FORTA) on pharmacotherapy and clinical endpoints--a pilot randomized controlled study. Eur J Clin Pharmacol 70 (10), 1261-1267 
Miller KE, Zylstra RG, Standridge JB (2000): The geriatric patient: a systematic approach to maintaining health. Am Fam Physician 61 (4), 1089-1104

Modreker MK, von Renteln-Kruse W (2009): Arzneimittel und Sturze im Alter. Internist $\underline{50}(4), 493-500$

Morello RT, Barker AL, Watts JJ, Haines T, Zavarsek SS, Hill KD, Brand C, Sherrington C, Wolfe R, Bohensky MA, Stoelwinder JU (2015): The extra resource burden of inhospital falls: a cost of falls study. Med J Aust $\underline{203}$ (9), 367

Moret C, Isaac M, Briley M (2008): Review. Problems associated with long-term treatment with selective serotonin reuptake inhibitors. J Psychopharmacol $\underline{23}$ (8), 967-974

Morley JE (2007): Falls--where do we stand? Missouri Med 104 (1), 63-67

Morley JE (2015): Dehydration, Hypernatremia, and Hyponatremia. Clin Geriatr Med $\underline{31}$ (3), 389-399

Morse JM, Black C, Oberle K, Donahue P (1989a): A prospective study to identify the fallprone patient. Soc Sci Med $\underline{28}$ (1), 81-86

Morse JM, Morse RM, Tylko SJ (1989b): Development of a Scale to Identify the FallProne Patient. Can J Aging $\underline{8}$ (4), 366-377

Morse JM, Faan A: Preventing patient falls. Establishing a fall intervention program. 2. Auflage; Springer New York, London 2008

Mosshammer D, Haumann H, Morike K, Joos S (2016): Polypharmacy-an Upward Trend with Unpredictable Effects. Dtsch Arztebl Int 113 (38), 627-633

Mouton CP, Bazaldua OV, Pierce B, Espino DV (2001): Common infections in older adults. Am Fam Physician 63 (2), 257-268

Muir SW, Gopaul K, Montero Odasso MM (2012): The role of cognitive impairment in fall risk among older adults: a systematic review and meta-analysis. Age Ageing 41 (3), 299-308

Müller R, Halfens R, Schwendimann R, Müller M, Imoberdorf R, Ballmer PE (2009): Risikofaktoren für Stürze und sturzbedingte Verletzungen im Akutspital - Eine retrospektive Fall-Kontroll-Studie. Pflege 22 (6), 431-441

Murphy J, Isaacs B (2004): The Post-Fall Syndrome. Gerontology 28 (4), 265-270

Neubart R: Der geriatrische Patient. In: Neubart R (Hrsg.): Repetitorium Geriatrie. Geriatrische Grundversorgung - Zusatz-Weiterbildung Geriatrie - Schwerpunktbezeichnung Geriatrie. 1. Aufl. 2015; Springer Berlin, Heidelberg, 9-14

Newman TB, Browner SB, Cummings SR, Steven R: Designing Case-Control Studies. In: Hulley SB, Cummings SR (Hrsg.): Designing Clinical Research. 4. Auflage; Lippincott Williams and Wilki, Philadelphia 2013, 97-117 
Nikolaus T (2001): Das geriatrische Assessment aktueller Erkenntnisstand hinsichtlich der Eignungskriterien (Diskrimination, Prädiktion, Evaluation, Praktikabilität). Z Gerontol Geriatr $\underline{34}(1), 36-42$

Nissen C, Frase L, Hajak G, Wetter TC (2014): Hypnotika--Stand der Forschung. Nervenarzt $\underline{85}$ (1), 67-76

Nobili A, Licata G, Salerno F, Pasina L, Tettamanti M, Franchi C, De Vittorio L, Marengoni A, Corrao S, Iorio A et al. (2011): Polypharmacy, length of hospital stay, and inhospital mortality among elderly patients in internal medicine wards. The REPOSI study. Eur J Clin Pharmacol 67 (5), 507-519

Nocera JR, Stegemöller EL, Malaty IA, Okun MS, Marsiske M, Hass CJ (2013): Using the Timed Up \& Go test in a clinical setting to predict falling in Parkinson's disease. Arch Phys Med Rehabil 94 (7), 1300-1305

O'Bryant SE, Humphreys JD, Smith GE, Ivnik RJ, Graff-Radford NR, Petersen RC, Lucas JA (2008): Detecting dementia with the mini-mental state examination in highly educated individuals. Arch Neurol $\underline{65}$ (7), 963-967

Oliver D, Britton M, Seed P, Martin FC, Hopper AH (1997): Development and evaluation of evidence based risk assessment tool (STRATIFY) to predict which elderly inpatients will fall: case-control and cohort studies. BMJ $\underline{315}$ (7115), 1049-1053

Oliver D, Hopper A, Seed, P (2000): Do hospital fall prevention programs work? A systematic review. J Am Geriatr Soc $\underline{48}$ (12), 1679-1689

Oliver D, Daly F, Martin FC, McMurdo MET (2004): Risk factors and risk assessment tools for falls in hospital in-patients: a systematic review. Age Ageing $\underline{33}$ (2), 122-130

Oliver D, Papaioannou A, Giangregorio L, Thabane L, Reizgys K, Foster G (2008): A systematic review and meta-analysis of studies using the STRATIFY tool for prediction of falls in hospital patients. How well does it work? Age Ageing $\underline{37}$ (6), 621-627

Olubodun JO, Ochs HR, von Moltke LL, Roubenoff R, Hesse LM, Harmatz JS, Shader RI, Greenblatt DJ (2003): Pharmacokinetic properties of zolpidem in elderly and young adults: possible modulation by testosterone in men. Br J Clin Pharmacol $\underline{56}$ (3), 297-304

O'Mahony D, O'Sullivan D, Byrne S, O'Connor MN, Ryan C, Gallagher P (2015): STOPP/START criteria for potentially inappropriate prescribing in older people: version 2. Age Ageing 44 (2), 213-218

Orimo H, Ito H, Suzuki T, Araki A, Hosoi T, Sawabe M (2006): Reviewing the definition of "elderly". Geriatr Gerontol Int $\underline{6}$ (3), 149-158

Pardasaney PK, Latham NK, Jette AM, Wagenaar RC, Ni P, Slavin MD, Bean JF (2012): Sensitivity to change and responsiveness of four balance measures for community-dwelling older adults. Phys Ther $\underline{92}$ (3), 388-397 
Park S (2018): Tools for assessing fall risk in the elderly: a systematic review and metaanalysis. Aging Clin Exp Res $\underline{30}$ (1),1-16

Park H, Satoh H, Miki A, Urushihara H, Sawada Y (2015): Medications associated with falls in older people: systematic review of publications from a recent 5-year period. Eur J Clin Pharamcol $\underline{71}$ (12), 1429-1440

Pasquetti P, Apicella L, Mangone G (2014): Pathogenesis and treatment of falls in elderly. Clin Cases Miner Bone Metab 11 (3), 222-225

Passaro A, Volpato S, Romagnoni F, Manzoli N, Zuliani G, Fellin R (2000): Benzodiazepines with different half-life and falling in a hospitalized population. J Clin Epidemiol $\underline{53}$ (12), 1222-1229

Payette MC, Bélanger C, Léveillé V, Grenier S (2016): Fall-Related Psychological Concerns and Anxiety among Community-Dwelling Older Adults: Systematic Review and MetaAnalysis. PloS One 11 (4), e0152848

Pearce N (2016): Analysis of matched case-control studies. BMJ 352, i969

Peduzzi P, Concato J, Kemper E, Holford TR, Feinstein AR (1996): A simulation study of the number of events per variable in logistic regression analysis. J Clin Epidemiol $\underline{49}$ (12), 1373-1379

Pereira J, Lawlor P, Vigano A, Dorgan M, Bruera E (2001): Equianalgesic dose ratios for opioids. a critical review and proposals for long-term dosing. J Pain Symptom Manage $\underline{22}$ (2), 672-687

Pétursson H (1994): The benzodiazepine withdrawal syndrome. Addiction $\underline{89}$ (11), 1455 1459

Podsiadlo, D.; Richardson, S. (1991): The timed "Up \& Go": a test of basic functional mobility for frail elderly persons. J Am Geriatr Soc $\underline{39}$ (2), 142-148

Prostran M, Vujović KS, Vučković S, Medić B, Srebro D, Divac N, Stojanović R, Vujović A, Jovanović L, Jotić A et al. (2016): Pharmacotherapy of Pain in the Older Population: The Place of Opioids. Front Aging Neurosci $\underline{8}, 144$

Psaty BM, Koepsell TD, Lin D, Weiss NS, Siscovick DS, Rosendaal FR, Pahor M, Furberg CD (1999): Assessment and control for confounding by indication in observational studies. JAm Geriatr Soc $\underline{47}$ (6), 749-754

Quach L, Yang FM, Berry SD, Newton E, Jones RN, Burr JA, Lipsitz LA (2013): Depression, antidepressants, and falls among community-dwelling elderly people: the MOBILIZE Boston study. J Gerontol A Biol Sci Med Sci $\underline{68}$ (12), 1575-1581

Rambhade S, Chakarborty A, Shrivastava A, Patil UK, Rambhade A (2012): A survey on polypharmacy and use of inappropriate medications. Toxicol Int $\underline{19}$ (1), 68-73 
Ramirez D, Wood RC, Becho J, Owings K, Markides K, Espino DV (2010): Mini-mental state exam domains predict falls in an elderly population: follow-up from the Hispanic Established Populations for Epidemiologic Studies of the Elderly (H-EPESE) study. Ethn Dis $\underline{20}(1), 48-52$

Rao A, Cohen HJ (2004): Symptom management in the elderly cancer patient. Fatigue, pain, and depression. J Natl Caner Inst Mongr 32, 150-157

Reidenberg MM, Levy M, Warner H, Coutinho CB, Schwartz MA, Yu G, Cheripko J (1978): Relationship between diazepam dose, plasma level, age, and central nervous system depression. Clin Pharmacol Ther 23 (4), 371-374

Renneboog B, Musch W, Vandemergel X, Manto MU, Decaux G (2006): Mild chronic hyponatremia is associated with falls, unsteadiness, and attention deficits. Am J Med $\underline{119}$ (1), 71.e1-8

Rhalimi M, Helou R, Jaecker P (2009): Medication use and increased risk of falls in hospitalized elderly patients. A retrospective, case-control study. Drugs Aging 26 (10), 847-852

Richardson K, Bennett K, Kenny RA (2015): Polypharmacy including falls risk-increasing medications and subsequent falls in community-dwelling middle-aged and older adults. Age Ageing 44 (1), 90-96

Richelson E (1997): Pharmacokinetic drug interactions of new antidepressants. A review of the effects on the metabolism of other drugs. Mayo Clin Proc 72 (9), 835-847

Rigler SK, Shireman TI, Cook-Wiens GJ, Ellerbeck EF, Whittle JC, Mehr DR, Mahnken JD (2013): Fracture risk in nursing home residents initiating antipsychotic medications. J Am Geriatr Soc 61 (5), 715-722

Rizzo JA, Friedkin R, Williams CS, Nabors J, Acampora D, Tinetti ME (1998): Health care utilization and costs in a Medicare population by fall status. Med Care $\underline{36}$ (8), 1174-1188

Rochon PA, Gurwitz JH (1997): Optimising drug treatment for elderly people. The prescribing cascade. BMJ $\underline{315}$ (7115), 1096-1099

Rodenburg EM, Hoorn EJ, Ruiter R, Lous JJ, Hofman A, Uitterlinden AG, Stricker BH, Visser LE (2013): Thiazide-associated hyponatremia: a population-based study. Am J Kidney Dis $\underline{62}(1), 67-72$

Rose S, van der Laan MJ (2009): Why match? Investigating matched case-control study designs with causal effect estimation. Int J Biostat $\underline{5}(1), 1$

Rosen, Sonja L, Reuben, David B. (2011): Geriatric assessment tools. Mt Sinai J Med $\underline{78}$ (4), 489-497

Rowe TA, Juthani-Mehta M (2013): Urinary tract infection in older adults. Aging Health $\underline{9}$ (5), 10.2217/ahe.13.38 
Rubenstein LZ (2006): Falls in older people: epidemiology, risk factors and strategies for prevention. Age Ageing $\underline{35}$ (2), 37-41

Rubenstein LZ, Robbins AS, Josephson KR, Schulman BL, Osterweil D (1990): The value of assessing falls in an elderly population. A randomized clinical trial. Ann Intern Med $\underline{113}$ (4), 308-316

Runciman WB, Roughead EE, Semple SJ, Adams RJ (2003): Adverse drug events and medication errors in Australia. Int J Qual Health Care 15 (1), 49-59

Russo M, Iasimone L, Ambrosino E (2002): Terapia antibiotica nelle infezioni batteriche dell' anziano. Aspetti generali e problematiche. Infez Med 10 (4), 204-212

S2e-Hausärztliche Leitlinie Multimedikation. S2e- Leitlinie 2013. DEGAM, 1-104

S3-Leitlinie Demenzen (2016). DGPPN u. DGN, 1-134

S3-Leitlinie Nicht erholsamer Schlaf/Schlafstörungen (2017). Somnologie 21 (1), 2-44

Salgado R, Lord SR, Packer J, Ehrlich F (1994): Factors associated with falling in elderly hospital patients. Gerontology $\underline{40}$ (6), 325-331

Sanders KM, Stuart AL, Williamson EJ, Simpson JA, Kotowicz MA, Young D, Nicholson, GC (2010): Annual high-dose oral vitamin D and falls and fractures in older women: a randomized controlled trial. JAMA $\underline{303}$ (18), 1815-1822

Sansone RA, Sansone LA(2014): Serotonin norepinephrine reuptake inhibitors. A pharmacological comparison. Innov Clin Neurosci 11 (3-4), 37-42

Sartini M, Cristina ML, Spagnolo AM, Cremonesi P, Costaguta C, Monacelli F, Garau J, Odetti P (2010): The epidemiology of domestic injurious falls in a community dwelling elderly population. An outgrowing economic burden. Eur J Public Health $\underline{20}$ (5), 604-606

Sass AC, Wurm S, Scheidt-Nave C (2010): Alter und Gesundheit: Eine Bestandsaufnahme aus Sicht der Gesundheitsberichterstattung. Bundesgesundheitsblatt, Gesundheitsforschung, Gesundheitsschutz $\underline{53}$ (5), 404-416

Schatzberg AF, Kremer C, Rodrigues HE, Murphy GM (2002): Double-Blind, Randomized Comparison of Mirtazapine and Paroxetine in Elderly Depressed Patients. Am J Geriatr Psychiatry 10 (5), 541-550

Schell W (2009): Sturz mit Folgen. Heilberufe 61 (12), 50-51

Schwab CW (1992): Trauma in the Geriatric Patient. Arch Surg 127 (6), 701

Schwendimann R, de Geest S, Milisen K (2006a): Evaluation of the Morse Fall Scale in hospitalised patients. Age Ageing 35 (3), 311-313

Schwendimann R, Bühler H, de Geest S, Milisen K (2006b): Falls and consequent injuries in hospitalized patients: effects of an interdisciplinary falls prevention program. BMC Health Serv Res $\underline{6}, 69$ 
Segev-Jacubovski O, Herman T, Yogev-Seligmann G, Mirelman A, Giladi N, Hausdorff JM (2011): The interplay between gait, falls and cognition: can cognitive therapy reduce fall risk? Expert Rev Neurother 11 (7), 1057-1075

Shewart WA, Wilks SS: Model-Building Strategies and Methods for Logistic Regression. In: Hosmer DW, Lemeshow S (Hrsg.): Applied logistic regression. 2. Auflage; Wiley Hoboken, New Jersey 2005, 91-142

Shorr RI, Mion LC, Chandler AM, Rosenblatt LC, Lynch D, Kessler LA (2008): Improving the capture of fall events in hospitals: combining a service for evaluating inpatient falls with an incident report system. J Am Geriatr Soc $\underline{56}$ (4), 701-704

Shuto H, Imakyure O, Matsumoto J, Egawa T, Jiang Y, Hirakawa M, Kataoka Y, Yanagawa $\mathrm{T}$ (2010): Medication use as a risk factor for inpatient falls in an acute care hospital. A casecrossover study. Br J Clin Pharmacol 69 (5), 535-542

Siebert S, Elkeles B, Hempel G, Kruse J, Smollich M (2013): Die PRISCUS-Liste im klinischen Test. Praktikabilität und Vergleich mit internationalen PIM-Listen. Z Gerontol Geriatr $\underline{46}(1), 35-47$

Skelly AC, Dettori JR, Brodt ED (2012): Assessing bias. The importance of considering confounding. Evid Based Spine Care J $\underline{3}$ (1), 9-12

Sommeregger U, Iglseder B, Böhmdorfer B, Benvenuti-Falger U, Dovjak P, Lechleitner M, Otto R, Roller RE, Gosch M (2010): Polypharmazie und Stürze im Alter. Wien Med Wochenschr $\underline{160}$ (11-12), 293-296

Song JW, Chung KC (2010): Observational studies: cohort and case-control studies. Plast Reconstr Surg 126 (6), 2234-2242

Spina E, Scordo MG (2002): Clinically significant drug interactions with antidepressants in the elderly. Drugs Aging 19 (4), 299-320

Stel VS, Smit JH, Pluijm SMG, Lips P (2003): Balance and mobility performance as treatable risk factors for recurrent falling in older persons. J Clin Epidemiol $\underline{56}$ (7), 659-668 Stevens JA, Corso PS, Finkelstein EA, Miller TR (2006): The costs of fatal and non-fatal falls among older adults. Inj Prev 12 (5), 290-295

Stockburger M, Möckel M: Bradyarrhythmien. In: Dietel M, Suttorp N, Zeitz M (Hrsg.): Harrisons Innere Medizin. Band 2., 18. Auflage; ABW Wissenschaftsverlag GmbH, Berlin 2012, 2003-2014

Stone CA, Lawlor PG, Savva GM, Bennett K, Kenny RA (2012): Prospective study of falls and risk factors for falls in adults with advanced cancer. J Clin Oncol $\underline{30}$ (17), 2128-2133

Tamiya H, Yasunaga H, Matusi H, Fushimi K, Ogawa S, Akishita M (2015): Hypnotics and the Occurrence of Bone Fractures in Hospitalized Dementia Patients. A Matched Case-Control Study Using a National Inpatient Database. PloS One 10 (6), e0129366 
Tan PN, Kumar V, Srivastava J (2004): Selecting the right objective measure for association analysis. Inf Syst 29 (4), 293-313

Tang N, Wu Y, Ma J, Wang B, Yu R (2010): Coffee consumption and risk of lung cancer. A meta-analysis. Lung Cancer $\underline{67}$ (1), 17-22

The Digitalis Investigation Group (1997): The effect of digoxin on mortality and morbidity in patients with heart failure. N Engl J Med $\underline{336}$ (8), 525-533

Tideiksaar R (1996): Preventing falls: how to identify risk factors, reduce complications. Geriatrics $\underline{51}$ (2), 43-6, 49-50, 53, quiz 54-5

Tinetti ME (1986): Performance-oriented assessment of mobility problems in elderly patients. J Am Geriatr Soc $\underline{34}$ (2), 119-126

Tinetti ME (2003): Clinical practice. Preventing falls in elderly persons. N Engl J Med $\underline{348}$ (1), 42-49

Tinetti ME, Speechley M, Ginter SF (1988): Risk factors for falls among elderly persons living in the community. N Engl J Med $\underline{319}$ (26), 1701-1707

Tinetti ME, Han L, Lee DSH, McAvay GJ, Peduzzi P, Gros CP, Zhou B, Lin H (2014): Antihypertensive medications and serious fall injuries in a nationally representative sample of older adults. JAMA Intern Med $\underline{174}$ (4), 588-595

Tombaugh TN, McIntyre NJ (1992): The mini-mental state examination: a comprehensive review. J Am Geriatr Soc 40 (9), 922-935

Trueblood PR, Hodson-Chennault N, McCubbin A, Youngclarke D (2001): Performance and Impairment-Based Assessments Among Community Dwelling Elderly. J Geriatr Phys Ther 24 (1), 2-6

Tzeng HM, Yin CY (2008): The extrinsic risk factors for inpatient falls in hospital patient rooms. J Nurs Care Qual 23 (3), 233-241

Urichuk L, Prior TI, Dursun S, Baker G (2008): Metabolism of atypical antipsychotics. Involvement of cytochrome p450 enzymes and relevance for drug-drug interactions. Curr Drug Metab $\underline{9}$ (5), 410-418

Uzun S, Kozumplik O, Jakovljević M, Sedić B (2010): Side effects of treatment with benzodiazepines. Psychiatr Danub 22 (1), 90-93

Vamos M, Erath JW, Hohnloser SH (2015): Digoxin-associated mortality. A systematic review and meta-analysis of the literature. Eur Heart J $\underline{36}$ (28), 1831-1838

van den Bussche H, Koller D, Kolonko T, Hansen H, Wegscheider K, Glaeske G, von Leitner EC, Schäfer I, Schön G (2011): Which chronic diseases and disease combinations are specific to multimorbidity in the elderly? Results of a claims data based cross-sectional study in Germany. BMC Public Health $\underline{11}, 101$ 
van der Velde N, Stricker BHC, Pols HAP, van der Cammen TJM (2007): Risk of falls after withdrawal of fall-risk-increasing drugs: a prospective cohort study. Br J Clin Pharmacol 63 (2), 232-237

van Doorn C, Gruber-Baldini AL, Zimmerman S, Hebel JR, Port CL, Baumgarten M, Quinn CC, Taler G, May C, Magaziner J et al. (2003): Dementia as a Risk Factor for Falls and Fall Injuries Among Nursing Home Residents. J Am Geriatr Soc 51 (9), 1213-1218 van Leeuwen RWF, Swart EL, Boven E, Boom FA, Schuitenmaker MG, Hugtenburg JG (2011): Potential drug interactions in cancer therapy. A prevalence study using an advanced screening method. Ann Oncol $\underline{22}$ (10), 2334-2341

van Rensbergen G, Nawrot T (2010): Medical conditions of nursing home admissions. BMC Geriatr $\underline{10}, 46$

van Schoor NM, Smit JH, Pluijm SMF, Jonker C, Lips P (2002): Different cognitive functions in relation to falls among older persons. Immediate memory as an independent risk factor for falls. J Clin Epidemiol $\underline{55}$ (9), 855-862

Vasilevskis EE, Han JH, Hughes CG, Ely EW (2012): Epidemiology and risk factors for delirium across hospital settings. Best Pract Res Clin Anesthesiol 26 (3), 277-287

Vetter NJ, Ford D (1989): Anxiety and depression scores in elderly fallers. Int J Geriatr Psychiatry 4 (3), 159-163

Victorri-Vigneau C, Dailly E, Veyrac G, Jolliet P (2007): Evidence of zolpidem abuse and dependence: results of the French Centre for Evaluation and Information on Pharmacodependence (CEIP) network survey. Br J Clin Pharmacol 64 (2), 198-209 von Renteln-Kruse W (2004): Stürze älterer Menschen. Diagnostik und Therapie. Dtsch Med Wochenschr 129 (16), 880-883

von Renteln-Kruse W, Krause T (2004): Sturzereignisse stationarer geriatrischer Patienten. Ergebnisse einer 3-jahrigen prospektiven Erfassung. Z Gerontol Geriatr $\underline{37}$ (1), 9-14 von Renteln-Kruse W, Micol W, Oster P, Schlierf G (1998): Arzneimittelverordnungen, Schwindel und Stürze bei über 75jährigen Krankenhauspatienten. Z Gerontol Geriatr $\underline{31}$ (4), 286-289

Vora MB, Trivedi HR, Shah BK, Tripathi CB (2011): Adverse drug reactions in inpatients of internal medicine wards at a tertiary care hospital. A prospective cohort study. J Pharmacol Pharmacother $\underline{2}(1), 21-25$

Vu MQ, Weintraub N, Rubenstein LZ (2004): Falls in the nursing home: are they preventable? J Am Med Dir Assoc $\underline{5}$ (6), 401-406

Wacholder S (1995): Design issues in case-control studies. Stat Methods Med Res 4 (4), 293-309 
Walker PC, Alrawi A, Mitchell JF, Regal RE, Khanderia U (2005): Medication use as a risk factor for falls among hospitalized elderly patients. Am J Health Syst Pharm $\underline{62}$ (23), 24952499

Wallace E, Salisbury C, Guthrie B, Lewis C, Fahey t, Smith SM (2015): Managing patients with multimorbidity in primary care. BMJ $\underline{350}$, h176

Wang PS, Bohn RL, Glynn RJ, Mogun H, Avorn J (2001a): Hazardous benzodiazepine regimens in the elderly. Effects of half-life, dosage, and duration on risk of hip fracture. Am J Psychiatry 158 (6), 892-898

Wang PS, Bohn RL, Glynn RJ, Mogun H, Avorn J (2001b): Zolpidem use and hip fractures in older people. J Am Geriatr Soc 49 (12), 1685-1690

Wang PS, Schneeweiss S, Avorn J, Fischer MA, Mogun H, Solomon DH, Brookhart MA (2005): Risk of death in elderly users of conventional vs. atypical antipsychotic medications. N Engl J Med $\underline{353}$ (22), 2335-2341

Wehling M (2012): Medikation im Alter. Kognitionseinschränkende Pharmaka. Internist $\underline{53}$ (10), 1240-1247

Weiner DK, Hanlon JT, Studenski SA (1998): Effects of Central Nervous System Polypharmacy on Falls Liability in Community-Dwelling Elderly. Gerontology 44 (4), 217 221

Werner H, Kuntsche J (2000): Infektionen im Alter--was ist anders? Z Gerontol Geriatr $\underline{33}$ (5), 350-356

Wickop B, Härterich S, Sommer C, Daubmann A, Baehr M, Langebrake C (2016): Potentially Inappropriate Medication Use in Multimorbid Elderly Inpatients: Differences Between the FORTA, PRISCUS and STOPP Ratings. Drugs Real World Outcomes $\underline{3}$ (3), $317-325$

Wickop B, Langebrake C (2014): Gute Verordnungspraxis bei älteren Patienten. Ther Um$\operatorname{sch} \underline{71}(6), 366-373$

Wilcoxon F (1947): Probability Tables for Individual Comparisons by Ranking Methods. Biometrics $\underline{3}(3), 119$

Wildes Tm, Dua P, Fowler SA, Miller JP, Carpenter CR, Avidan MS, Stark S (2015): Systematic review of falls in older adults with cancer. J Geriatr Oncol $\underline{6}$ (1),70-83

Williams TA, King G, Hill AM, Rajagopal M, Barnes T, Basu A, Pascoe G, Birkett K, Kidd H (2007): Evaluation of a falls prevention programme in an acute tertiary care hospital. J Clin Nurs $\underline{16}$ (2), 316-324

Wirtz M, Ulrich R: Bivariate deskriptive Statistik. Holling H, Schmitz B (Hrsg.): Handbuch Statistik, Methoden und Evaluation. 1. Aufl.; Hogrefe, Göttingen 2010, 288-302 
Woolcott JC, Richardson KJ, Wiens MO, Patel B, Marin J, Khan KM, Marra CA (2009): Meta-analysis of the impact of 9 medication classes on falls in elderly persons. Arch Intern Med $\underline{169}$ (21), 1952-1960

Yang Y, van Schooten KS, Sims-Gould J, McKay HA, Feldman F, Robinovitch SN (2017): Sex differences in the circumstances leading to falls. Evidence from real-life falls captured on video in long-term care. J Am Med Dir Assoc 26. pii:S1525-8610(17)30478-4

Yokoya T, Demura S, Sato S (2007): Relationships between physical activity, ADL capability and fall risk in community-dwelling Japanese elderly population. Environ Health Prev Med 12 (1), 25-32

Zhang Y, Zhou XH, Meranus DH, Wang L, Kukull WA (2016): Benzodiazepine Use and Cognitive Decline in Elderly With Normal Cognition. Alzheimer Dis Assoc Disord 30 (2), 113-117

Ziff OJ, Lane DA, Samra M, Griffith M, Kirchhof P, Lip GHY, Steeds RP, Towned J, Kotecha D (2015): Safety and efficacy of digoxin. Systematic review and meta-analysis of observational and controlled trial data. BMJ $\underline{351}$, h4451

Zijlstra GAR, van Haastregt JCM, van Eijk JTM, van Rossum E, Stalenhoef PA, Kempen, GIJM (2007): Prevalence and correlates of fear of falling, and associated avoidance of activity in the general population of community-living older people. Age Ageing $\underline{36}$ (3), 304309

ZumBrunnen TL, Jann MW (1998): Drug Interactions with Antipsychotic Agents. CNS Drugs $\underline{9}$ (5), 381-401 


\section{Danksagung}

Ich danke Herrn Professor Dr. med. Roland Nau, Chefarzt der geriatrischen Abteilung des Evangelischen Krankenhaus Göttingen-Weende, für die Überlassung des Themas und die freundliche Unterstützung bei der Erstellung meiner Arbeit sowie Herrn Prof. Wolfgang Himmel für die Unterstützung bei der statistischen Auswertung. 